Department of Policy and Planning Sciences

\author{
Discussion Paper Series
}

No.1358

Higher-order bias corrections for kernel type

density estimators on the unit or semi-infinite interval

by

Gaku IGARASHI and Yoshihide KAKIZAWA

Apr 2019

UNIVERSITY OF TSUKUBA

Tsukuba, Ibaraki 305-8573

JAPAN 


\title{
Higher-order bias corrections for kernel type density estimators on the unit or semi-infinite interval
}

Gaku Igarashi $^{1}$ and Yoshihide Kakizawa ${ }^{2}$

${ }^{1}$ Department of Policy and Planning Sciences, Faculty of Engineering, Information and Systems, University of Tsukuba, 1-1-1 Tennodai, Tsukuba, Ibaraki 305-8573, Japan ${ }^{2}$ Faculty of Economics, Hokkaido University, Nishi 7, Kita 9, Kita-ku, Sapporo 060-0809, Japan

\begin{abstract}
For the data of size $n$ from the unit or semi-infinite interval, several asymmetric kernel density estimators (KDEs), having the mean integrated squared errors (MISEs) of order $O\left(n^{-4 / 5}\right)$ or $O\left(n^{-8 / 9}\right)$, have been studied over the last two decades. In this paper, we develop more higher-order bias-corrected asymmetric KDEs, achieving the order $O\left(n^{-4 p /(4 p+1)}\right)$, where $p \geq 2$ is a given integer; these higher-order bias correction methods can be also applied to the classical Rosenblatt-Parzen KDEs. We illustrate the finite sample performance of the higher-order bias-corrected asymmetric KDEs through the simulations.
\end{abstract}

Keywords: nonparametric density estimation; boundary bias problem; asymmetric kernel; higher-order bias correction;

MSC: $62 \mathrm{G} 07 ; 62 \mathrm{G} 20$

\section{Introduction}

The kernel density estimator $(\mathrm{KDE}), \widehat{f}_{h}^{(K)}(x)=(n h)^{-1} \sum_{i=1}^{n} K\left(\left(x-X_{i}\right) / h\right)$, developed by Rosenblatt (1956) and Parzen (1962), is a popular nonparametric estimator, where $\left\{X_{1}, \ldots, X_{n}\right\}$ is a random sample drawn from an unknown density $f$ with support $\mathbb{R}, h>0$ is a bandwidth, and $K$ is a symmetric kernel. If $f$ is $2 p$ times continuously differentiable for some $p \in \mathbb{N}$, using a $2 p$ th-order kernel $K_{[2 p]}$, i.e., $\int_{-\infty}^{\infty} K_{[2 p]}(s) d s=1, \int_{-\infty}^{\infty} s^{\ell} K_{[2 p]}(s) d s=0, \ell=1, \ldots, 2 p-1$, and $\int_{-\infty}^{\infty} s^{2 p} K_{[2 p]}(s) d s \neq 0$, the bias and variance of the $2 p$ th-order $\operatorname{KDE} \widehat{f}_{h}^{\left(K_{[2 p]}\right)}$ are $O\left(h^{2 p}\right)$ and $O\left(n^{-1} h^{-1}\right)$, respectively, hence, with $h \propto n^{-1 /(4 p+1)}$, the mean squared error (MSE) and mean integrated squared error (MISE) are $O\left(n^{-4 p /(4 p+1)}\right)$. The use of higher-order kernels enables us to get the faster convergence rate of the M(I)SE. Schucany and Sommers (1977) and Jones and Foster (1993) addressed how to generate a reasonable $K_{[4]}$ from a given $K_{[2]}$, in a variety of ways. One attractive and simple answer is to produce a class of the fourth-order kernels

$$
K_{[4],(1, a)}(s)= \begin{cases}\frac{1}{1-a^{2}}\left\{K_{[2]}(s)-a^{3} K_{[2]}(a s)\right\}, & a \neq 1, \\ \frac{1}{2}\left\{3 K_{[2]}(s)+s K_{[2]}^{\prime}(s)\right\}, & a=1 .\end{cases}
$$

Email: g-igarashi@sk.tsukuba.ac.jp (G. Igarashi), kakizawa@econ.hokudai.ac.jp (Y. Kakizawa).

The authors reported some preliminary results at the Japanese Joint Statistical Meeting (2018, September) and the Mathematical Society of Japan Autumn Meeting (2018, September).

The first author has been supported by the Japan Society for the Promotion of Science (JSPS); Grant-inAid for Young Scientists (B) [Grant Number 17K13714]. The second author has been supported by the JSPS; Grant-in-Aid for Scientific Research (C) [Grant Number 17K00041]. 
However, by definition, the $2 p$ th-order $\operatorname{KDE} \widehat{f}_{h}^{\left(K_{[2 p]}\right)}$ necessarily loses the nonnegativity unless $p=1$, so that nonnegative bias correction methods were discussed by Terrell and Scott (1980), Jones and Foster (1993), and Jones et al. (1995).

Unfortunately, if $\operatorname{supp}(f) \neq \mathbb{R}$, the classical Rosenblatt-Parzen KDE has, in general, the boundary bias which is $O(1)$ near the boundary of $\operatorname{supp}(f)$. Various remedies were studied, e.g., renormalization, reflection, generalized jackknifing (Jones (1993)), transformation (Marron and Ruppert (1994)), and advanced reflection (Zhang et al. (1999)). On the other hand, instead of the location-scale type $K((x-\cdot) / h) / h$, applying several asymmetric kernels whose support match $\operatorname{supp}(f)$ has attracted considerable attention over the last two decades. Among many papers are Chen (1999, 2000), Jin and Kawczak (2003), Scaillet (2004), Marchant et al. (2013), Saulo et al. (2013), Igarashi and Kakizawa (2014b), Igarashi (2016b), Kakizawa and Igarashi (2017), and Kakizawa (2018). Note that "good" asymmetric KDEs have the MISEs of order $O\left(n^{-4 / 5}\right)$. To achieve the order $O\left(n^{-8 / 9}\right)$, some bias correction methods have been further discussed in recent years, even when $\operatorname{supp}(f)=[0,1]$ or $[0, \infty)$. See Hirukawa (2010; correction 2016), Leblanc (2010), Hirukawa and Sakudo (2014, 2015), Igarashi and Kakizawa (2014a, 2015, 2018a,b,c), Igarashi (2016a), and Zougab and Adjabi (2016).

The objective of this paper is to develop more higher-order bias-corrected density estimation, by generalizing novel ideas of the bias correction methods due to Schucany and Sommers (1977), Terrell and Scott (1980), and Jones and Foster (1993). In Section 2, we describe basic asymptotic properties of the asymmetric KDE (without bias corrections). In Section 3, we establish that the proposed higher-order bias-corrected asymmetric KDEs attain the convergence rate $n^{-4 p /(4 p+1)}$ of the MISE. It is revealed, however, that, for some asymmetric KDEs, the convergence rates after the bias corrections are at most $n^{-\left(4 p^{*}+2\right) /\left(4 p^{*}+3\right)}$, with an integer $p^{*}$. In Section 4 , we provide examples of kernels with support $[0, \infty)$ or $[0,1]$. Section 5 presents simulation studies to illustrate the finite sample performance of the bias-corrected estimators. Some general comments are given in Section 6. The proofs are postponed to the Appendix.

Notation The dependency on the sample size $n$ is suppressed (e.g., the smoothing parameter is denoted by $\beta$, instead of $\beta_{n}$ ), but, unless otherwise stated, the limits will be taken as $n \rightarrow \infty$. We write $\mathcal{S}=\operatorname{supp}(f)$ for simplicity, and, as usual, use the notation $\|h\|_{\mathcal{S}}=\sup _{x \in \mathcal{S}}|h(x)|$ for any bounded function $h$ on $\mathcal{S}$. We denote by $h^{(j)}(x)=(d / d x)^{j} h(x)$ the $j$ th derivative of $h$ (if it exists), and write $h^{(0)}(x)=h(x)$. Further, $\chi_{A}$ denotes the indicator function of a set $A$, and $\lceil y\rceil$ denotes the smallest integer greater than or equal to $y$. Conventionally, the empty sum (e.g., $\left.\sum_{k=1}^{0}\right)$ is defined to equal zero. The bias, MSE, and MISE for an estimator $\widehat{f}(x)$ of $f(x)$, $x \in \mathcal{S}$, are denoted by $\operatorname{Bias}[\widehat{f}(x)]=E[\widehat{f}(x)]-f(x), M S E[\widehat{f}(x)]=E\left[\{\widehat{f}(x)-f(x)\}^{2}\right]$, and $M I S E[\widehat{f}]=\int_{\mathcal{S}} M S E[\widehat{f}(x)] d x$. 


\section{Preliminaries}

We assume that $\mathcal{X}^{(n)}=\left\{X_{1}, \ldots, X_{n}\right\}$ is a random sample drawn from an unknown density $f$ with support $\mathcal{S}=[0, \infty)$ or $[0,1]$. Let $\beta>0$ be a smoothing parameter, such that $\beta \rightarrow 0$ and $n \beta \rightarrow \infty$, unless otherwise stated. We construct an estimator in the form of

$$
\widehat{f}_{\beta}(x)=\frac{1}{n} \sum_{i=1}^{n} K\left(X_{i} ; x, \beta\right), \quad x \in \mathcal{S}
$$

(referred to as an asymmetric $\mathrm{KDE}$ throughout this paper), where $K(\cdot ; x, \beta)(\geq 0)$ is a density with support $\mathcal{S}$, such that the kernel $K(s ; x, \beta)$ concentrates around $s=x$ as $\beta \rightarrow 0$.

Before proceeding to complete description of assumptions, we briefly mention what kinds of assumptions are required here. According to our previous works (e.g., Igarashi and Kakizawa $(2015,2018 \mathrm{a}, \mathrm{c})$ and Igarashi $(2016 \mathrm{a}))$, additional properties on $K(\cdot ; x, \beta)$, i.e.,

- the uniform/nonuniform bounds of $\sup _{s \in \mathcal{S}} K(s ; x, \beta)$,

- the tractability of the product kernel $K\left(s ; x, \beta / a_{0}\right) K\left(s ; x, \beta / a_{0}^{\prime}\right)$ for any $a_{0}, a_{0}^{\prime}>0$, and

- when $\mathcal{S}=[0, \infty)$, the asymptotic behaviour of $\int_{\beta^{-\tau}}^{\infty} K(s ; x, \beta) d x$ for any $\tau \in(0,1)$

(Assumptions A1-A3) are indispensable. The $j$ th moment around $x \in \mathcal{S}$ is denoted by

$$
\mu_{j}(K(\cdot ; x, \beta))=\int_{\mathcal{S}}(s-x)^{j} K(s ; x, \beta) d s \quad \text { (if it exists) } .
$$

Note that $\mu_{0}(K(\cdot ; x, \beta)) \equiv 1$, since the chosen kernel is a certain density with support $\mathcal{S}$. The results in this paper heavily depend on the moments up to the $2(p+1)$ th order, for some $p \in \mathbb{N}$ (Assumption $\mathrm{A} 4[p])$, under which $\mu_{j}(K(\cdot ; x, \beta)), x \in \mathcal{S}$, is expanded as a power of $\beta$. The regularity on the density $f$ to be estimated (Assumption $\mathrm{A} 5[p](\mathrm{i}, \mathrm{ii})$ or $\mathrm{A} 5^{\prime}(\mathrm{i})$ ) is standard in nonparametric density estimation. It should be remarked that Assumption A3, together with the latter part of $\mathrm{A} 5[p]$ (iii) (or $\mathrm{A} 5^{\prime}(\mathrm{ii})$ ), is somewhat technical, but will be used only for the approximations of the integrated squared bias/variance when $\mathcal{S}=[0, \infty)$.

\subsection{Assumptions}

Throughout this paper, we denote by

$$
\mathcal{S}_{I}=\left\{\begin{array}{ll}
(0, \infty), & \mathcal{S}=[0, \infty), \\
(0,1), & \mathcal{S}=[0,1]
\end{array} \text { and } \quad \mathcal{S}_{B}= \begin{cases}\{0\}, & \mathcal{S}=[0, \infty) \\
\{0,1\}, & \mathcal{S}=[0,1]\end{cases}\right.
$$

the interior and boundary, respectively, of $\mathcal{S}$. We often distinguish between the two cases of a set of points far away from $\mathcal{S}_{B}$ and a set of points near $\mathcal{S}_{B}$, i.e.,

$$
\begin{aligned}
\mathcal{S}_{I, \beta}= \begin{cases}\left\{x \in \mathcal{S} \mid \frac{x}{\beta} \rightarrow \infty\right\}, & \mathcal{S}=[0, \infty), \\
\left\{x \in \mathcal{S} \mid \frac{x}{\beta} \rightarrow \infty, \frac{1-x}{\beta} \rightarrow \infty\right\}, & \mathcal{S}=[0,1],\end{cases} \\
\mathcal{S}_{B, \beta, \kappa}= \begin{cases}\mathcal{S}_{0, \beta, \kappa}, & \mathcal{S}=[0, \infty), \\
\mathcal{S}_{0, \beta, \kappa} \cup \mathcal{S}_{1, \beta, \kappa}, & \mathcal{S}=[0,1],\end{cases}
\end{aligned}
$$


where $\mathcal{S}_{0, \beta, \kappa}=\{x \in \mathcal{S} \mid x / \beta \rightarrow \kappa\}$ and $\mathcal{S}_{1, \beta, \kappa}=\{x \in \mathcal{S} \mid(1-x) / \beta \rightarrow \kappa\}$ (here and subsequently, $\kappa \geq 0$ is a constant, unless otherwise stated). Also, we write

$$
\psi(x)=\left\{\begin{array}{ll}
x, & \mathcal{S}=[0, \infty), \\
x(1-x), & \mathcal{S}=[0,1]
\end{array} \text { and } \quad V(x ; f)=\frac{f(x)}{2 \sqrt{\pi \psi(x)}} .\right.
$$

Then, the following assumptions on $K(\cdot ; x, \beta)$ and $f$ are made for some $p \in \mathbb{N}$ :

A1. (i) $\sup _{x \in \mathcal{S}} \sup _{s \in \mathcal{S}} K(s ; x, \beta) \leq C_{K} \beta^{-1}$ for some constant $C_{K}>0$, independent of $\beta$.

(ii) When $x \in \mathcal{S}_{I}, \sup _{s \in \mathcal{S}} K(s ; x, \beta) \leq C_{K}^{\prime}\{\beta \psi(x)\}^{-1 / 2}$ for some constant $C_{K}^{\prime}>0$, independent of $\beta$ and $x$.

A2. For any constants $a_{0}, a_{0}^{\prime}>0$,

$$
\int_{\mathcal{S}} K\left(s ; x, \beta / a_{0}\right) K\left(s ; x, \beta / a_{0}^{\prime}\right) d s= \begin{cases}\left(\frac{2 a_{0} a_{0}^{\prime}}{a_{0}+a_{0}^{\prime}}\right)^{1 / 2} \frac{\beta^{-1 / 2}}{2 \sqrt{\pi \psi(x)}}+O\left(\beta^{1 / 2}\{\psi(x)\}^{-3 / 2}\right), & x \in \mathcal{S}_{I, \beta}, \\ \beta^{-1} \varsigma_{a_{0}, a_{0}^{\prime}}(\kappa)\left[1+\chi_{\left\{x \notin \mathcal{S}_{B}\right\}} O(1)\right], & x \in \mathcal{S}_{B, \beta, \kappa}\end{cases}
$$

for some function $\varsigma_{a_{0}, a_{0}^{\prime}}$, independent of $\beta$.

A3. When $\mathcal{S}=[0, \infty)$, for any constants $k>0$ and $\tau \in(0,1)$, and for all sufficiently small $\beta>0$,

$$
\int_{\beta^{-\tau}}^{\infty} K(s ; x, \beta) d x=O\left(\beta^{\tau(k+1)} s^{k+1}\right), \quad s>0 .
$$

A4[p]. The moments around $x \in \mathcal{S}$ admit asymptotic expansions, as follows: when $\mathcal{S}=[0, \infty)$,

$$
\mu_{j}(K(\cdot ; x, \beta))= \begin{cases}\sum_{k=\lceil j / 2\rceil}^{\min (j, p)} \zeta_{j, k} x^{j-k} \beta^{k}+\chi_{\{j>p\}} O\left(\beta^{p+1}(x+\beta)^{j-(p+1)}\right), & j=1, \ldots, 2 p, \\ O\left(\beta^{p+1}(x+\beta)^{p+1}\right), & j=2(p+1)\end{cases}
$$

for some constants $\zeta_{j, k}$ 's, independent of $\beta$ and $x$, whereas, when $\mathcal{S}=[0,1]$, uniformly in $x \in[0,1]$,

$$
\mu_{j}(K(\cdot ; x, \beta))= \begin{cases}\sum_{k=\lceil j / 2\rceil}^{p} \zeta_{j, k}(x) \beta^{k}+O\left(\beta^{p+1}\right), & j=1, \ldots, 2 p \\ O\left(\beta^{p+1}\right), & j=2(p+1)\end{cases}
$$

for some polynomials in $x ; \zeta_{j, k}(x)$ 's, independent of $\beta$, where $\zeta_{2,1}(x)=\psi(x)$.

A5[p]. (i) $f$ is $2 p$ times continuously differentiable on $\mathcal{S}$, with $\sum_{j=0}^{2 p}\left\|f^{(j)}\right\|_{\mathcal{S}}<\infty$.

(ii) $f^{(2 p)}$ is Hölder continuous on $\mathcal{S}$, i.e., there exist constants $\eta_{2 p} \in(0,1]$ and $L_{2 p}>0$, such that $\left|f^{(2 p)}(s)-f^{(2 p)}(t)\right| \leq L_{2 p}|s-t|^{\eta_{2 p}}$ for any $s, t \in \mathcal{S}$.

(iii) When $\mathcal{S}=[0, \infty), \sum_{j=p}^{2 p} \int_{0}^{\infty}\left\{x^{j-p} f^{(j)}(x)\right\}^{2} d x<\infty$ and there exists a constant $k_{2 p}>\left\{2 p(2 p+1)+(2 p-1) \eta_{2 p}\right\} / \eta_{2 p}$ such that $\int_{0}^{\infty} x^{k_{2 p}+1} f(x) d x<\infty$ (in this case, for any constant $\left.\tau_{2 p} \in\left(2 p /\left(k_{2 p}+1\right), \eta_{2 p} /\left(2 p+1+\eta_{2 p}\right)\right), \int_{0}^{\beta^{-\tau_{2 p}}} \beta^{\eta_{2 p}}\left(1+x^{p+\eta_{2 p} / 2}\right)^{2} d x=o(1)\right)$. 
Note that, in some cases, Assumptions A4[p] and A5[p] will be weakened, as follows:

$\mathrm{A} 4^{\prime}[J]$. When $\mathcal{S}=[0, \infty)$,

$$
\mu_{j}(K(\cdot ; x, \beta))=\sum_{k=\lceil j / 2\rceil}^{\min (j, J-1)} \zeta_{j, k} x^{j-k} \beta^{k}+\chi_{\{j>J-1\}} O\left(\beta^{J}(x+\beta)^{j-J}\right), \quad j=1, \ldots, 2 J
$$

for some constants $\zeta_{j, k}$ 's, independent of $\beta$ and $x$, whereas, when $\mathcal{S}=[0,1]$, uniformly in $x \in[0,1]$,

$$
\mu_{j}(K(\cdot ; x, \beta))=\sum_{k=\lceil j / 2\rceil}^{J-1} \zeta_{j, k}(x) \beta^{k}+O\left(\beta^{J}\right), \quad j=1, \ldots, 2 J
$$

for some polynomials in $x ; \zeta_{j, k}(x)$ 's, independent of $\beta$, where $\zeta_{2,1}(x)=\psi(x)$.

A $5^{\prime}$. (i) $f$ is continuously differentiable on $\mathcal{S}$, with $\|f\|_{\mathcal{S}}+\left\|f^{(1)}\right\|_{\mathcal{S}}<\infty$.

(ii) When $\mathcal{S}=[0, \infty)$, there exists a constant $k^{\prime}>0$, such that $\int_{0}^{\infty} x^{k^{\prime}+1} f(x) d x<\infty$.

\subsection{Asymptotic properties of asymmetric KDE (without bias corrections)}

In this subsection, the asymptotic properties of the asymmetric KDE (1) are presented.

Theorem 1 (i) Suppose that Assumptions A4[p] and A5[p](i,ii) hold for some $p \in \mathbb{N}$. Then,

$$
\operatorname{Bias}\left[\widehat{f}_{\beta}(x)\right]=\sum_{k=1}^{p} \beta^{k} \gamma_{k}(x ; f)+\mathcal{E}_{\beta, p}(x), \quad x \in \mathcal{S},
$$

where $\gamma_{k}(x ; f)$ and $\mathcal{E}_{\beta, p}(x)$ are given, as follows: when $\mathcal{S}=[0, \infty)$,

$$
\gamma_{k}(x ; f)=\sum_{j=k}^{2 k} \zeta_{j, k} x^{j-k} \frac{f^{(j)}(x)}{j !}, \quad \mathcal{E}_{\beta, p}(x)=O\left(\beta^{p+\eta_{2 p} / 2}(1+x)^{p+\eta_{2 p} / 2}\right),
$$

and, when $\mathcal{S}=[0,1]$,

$$
\gamma_{k}(x ; f)=\sum_{j=1}^{2 k} \zeta_{j, k}(x) \frac{f^{(j)}(x)}{j !}, \quad \mathcal{E}_{\beta, p}(x)=O\left(\beta^{p+\eta_{2 p} / 2}\right) \text { uniformly in } x \in[0,1] .
$$

(ii) Suppose that Assumptions A1, A2, A4'[1], and A5'(i) hold. Then,

$$
V\left[\widehat{f}_{\beta}(x)\right]= \begin{cases}n^{-1} \beta^{-1 / 2} V(x ; f)\left[1+O\left(\beta \psi^{-1}(x)\right)\right]+O\left(n^{-1}\right), & x \in \mathcal{S}_{I, \beta}, \\ n^{-1} \beta^{-1} f(x)\left[\varsigma_{1,1}(\kappa)+\chi_{\left\{x \notin \mathcal{S}_{B}\right\}} O(1)\right]+O\left(n^{-1}\right), & x \in \mathcal{S}_{B, \beta, \kappa} .\end{cases}
$$

(iii) Suppose that Assumption A1(i) holds. If $n \beta / \log n \rightarrow \infty$, then, $\widehat{f}_{\beta}(x)-E\left[\widehat{f}_{\beta}(x)\right] \stackrel{\text { a.s. }}{\rightarrow} 0, x \in \mathcal{S}$.

Remark 1 Under Assumptions A4'[1] and A5[1](i), we have (see also Remark A.1)

$$
\begin{aligned}
\text { when } \mathcal{S} & =[0, \infty), \operatorname{Bias}\left[\widehat{f}_{\beta}(x)\right]=O(\beta(1+x)), \\
\text { when } \mathcal{S} & =[0,1] \text {, uniformly in } x \in[0,1], \operatorname{Bias}\left[\widehat{f}_{\beta}(x)\right]=O(\beta) .
\end{aligned}
$$

Theorem 1(iii) immediately yields the strong consistency of the estimator (1);

$$
\widehat{f}_{\beta}(x) \stackrel{\text { a.s. }}{\rightarrow} f(x) \text { for fixed } x \in \mathcal{S} .
$$


Theorem 2 Suppose that Assumptions A1, A2, A4'[1], and A5'(i) hold. Then,

$$
\begin{gathered}
\left(n \beta^{1 / 2}\right)^{1 / 2}\left\{\widehat{f}_{\beta}(x)-E\left[\widehat{f}_{\beta}(x)\right]\right\} \stackrel{d}{\rightarrow} N(0, V(x ; f)) \quad \text { for fixed } x \in \mathcal{S}_{I}, \\
(n \beta)^{1 / 2}\left\{\widehat{f}_{\beta}(x)-E\left[\widehat{f}_{\beta}(x)\right]\right\} \stackrel{\stackrel{d}{\rightarrow}}{\rightarrow} N\left(0, \varsigma_{1,1}(0) f(x)\right) \quad \text { for } x \in \mathcal{S}_{B} .
\end{gathered}
$$

A replacement of $E\left[\widehat{f}_{\beta}(x)\right]$ by $f(x)$ (or $f(x)+\beta \gamma_{1}(x ; f)$ ) is a routine problem in density estimation theory (use Slutsky's lemma; see Theorems 1(i) and 2) ${ }^{[1]}$.

Theorem 1 shows that

$$
M S E\left[\widehat{f}_{\beta}(x)\right]=\left\{\begin{array}{l}
A M S E\left[\widehat{f}_{\beta}(x)\right]+o\left(\beta^{2}+n^{-1} \beta^{-1 / 2}\right) \quad \text { for fixed } x \in \mathcal{S}_{I}, \\
A M S E\left[\widehat{f}_{\beta}(x)\right]+o\left(\beta^{2}+n^{-1} \beta^{-1}\right) \quad \text { for } x \in \mathcal{S}_{B, \beta, \kappa},
\end{array}\right.
$$

where

$$
A M S E\left[\widehat{f}_{\beta}(x)\right]=\left\{\begin{array}{lr}
\beta^{2} \gamma_{1}^{2}(x ; f)+n^{-1} \beta^{-1 / 2} V(x ; f) & \text { for fixed } x \in \mathcal{S}_{I}, \\
\beta^{2} \gamma_{1}^{2}(x ; f)+n^{-1} \beta^{-1} \varsigma_{1,1}(\kappa) f(x) & \text { for } x \in \mathcal{S}_{B, \beta, \kappa} .
\end{array}\right.
$$

Note that

$$
\min _{\beta>0} A M S E\left[\widehat{f}_{\beta}(x)\right]=\left\{\begin{array}{l}
\frac{5}{4}\left[4 \gamma_{1}^{2}(x ; f)\left\{V(x ; f) n^{-1}\right\}^{4}\right]^{1 / 5} \text { for fixed } x \in \mathcal{S}_{I}, \\
\frac{3}{2}\left[2 \gamma_{1}^{2}(x ; f)\left\{\varsigma_{1,1}(\kappa) f(x) n^{-1}\right\}^{2}\right]^{1 / 3} \quad \text { for } x \in \mathcal{S}_{B, \beta, \kappa}
\end{array}\right.
$$

(we assume $\gamma_{1}(x ; f) \neq 0$ ). Although the estimator (1) has the slower convergence rate near the boundary $\mathcal{S}_{B}$, such a different rate is asymptotically negligible on the MISE.

Theorem 3 Suppose that Assumptions A1-A3, A4[1], and A5[1] hold. Then,

$$
\operatorname{MISE}\left[\widehat{f}_{\beta}\right]=A M I S E\left[\widehat{f}_{\beta}\right]+o\left(\beta^{2}+n^{-1} \beta^{-1 / 2}\right),
$$

where

$$
A M I S E\left[\widehat{f}_{\beta}\right]=\beta^{2} \int_{\mathcal{S}} \gamma_{1}^{2}(x ; f) d x+n^{-1} \beta^{-1 / 2} \int_{\mathcal{S}} V(x ; f) d x
$$

is minimized at

$$
\beta=\left[\frac{\int_{\mathcal{S}} V(x ; f) d x}{4 \int_{\mathcal{S}} \gamma_{1}^{2}(x ; f) d x} n^{-1}\right]^{2 / 5}
$$

when $\gamma_{1}(x ; f) \not \equiv 0$, that is,

$$
\min _{\beta>0} A M I S E\left[\widehat{f}_{\beta}\right]=\frac{5}{4}\left[4 \int_{\mathcal{S}} \gamma_{1}^{2}(x ; f) d x\left\{\int_{\mathcal{S}} V(x ; f) d x n^{-1}\right\}^{4}\right]^{1 / 5} .
$$

${ }^{[1]}$ Suppose that Assumptions A1, A2, A4[1] , and A5[1](i,ii) hold.

(i). If $n \beta^{5 / 2+\eta_{2}} \rightarrow 0$, then, for fixed $x \in \mathcal{S}_{I}$,

$$
\left(n \beta^{1 / 2}\right)^{1 / 2}\left\{\widehat{f}_{\beta}(x)-f(x)-\beta \gamma_{1}(x ; f)\right\} \stackrel{d}{\rightarrow} N(0, V(x ; f)),
$$

hence, if $n \beta^{5 / 2} \rightarrow 0$, then, $\left(n \beta^{1 / 2}\right)^{1 / 2}\left\{\widehat{f}_{\beta}(x)-f(x)\right\} \stackrel{d}{\rightarrow} N(0, V(x ; f))$.

(ii). If $n \beta^{3+\eta_{2}} \rightarrow 0$, then, for $x \in \mathcal{S}_{B}$,

$$
(n \beta)^{1 / 2}\left\{\widehat{f}_{\beta}(x)-f(x)-\beta \gamma_{1}(x ; f)\right\} \stackrel{d}{\rightarrow} N\left(0, \varsigma_{1,1}(0) f(x)\right),
$$

hence, if $n \beta^{3} \rightarrow 0$, then, $(n \beta)^{1 / 2}\left\{\widehat{f}_{\beta}(x)-f(x)\right\} \stackrel{d}{\rightarrow} N\left(0, \varsigma_{1,1}(0) f(x)\right)$. 


\section{Additive, TS-type, and JF-type bias corrections}

The main contribution of this paper is to study higher-order extensions of the previous works (e.g., Igarashi and Kakizawa (2015, 2018a) and Igarashi (2016a)). From now on, let $p \in \mathbb{N} \backslash\{1\}$, unless otherwise stated. Given a positive vector $\boldsymbol{a}=\left(a_{1}, \ldots, a_{p}\right)^{\prime}$, such that the $a_{k}$ 's are distinct, the additive, TS-type, and JF-type bias-corrected KDEs of $f(x), x \in \mathcal{S}$, are defined by

$$
\begin{aligned}
\widehat{f}_{\beta, A D D_{\boldsymbol{a}}^{p}}(x) & =\sum_{k=1}^{p} c_{k}(\boldsymbol{a}) \widehat{f}_{\beta / a_{k}}(x)=\frac{1}{n} \sum_{i=1}^{n} K_{A D D_{\boldsymbol{a}}^{p}}\left(X_{i} ; x, \beta\right) \quad(\text { say }) \\
\widehat{f}_{\beta, T S_{\boldsymbol{a}}^{p}}(x) & =\exp \left[\sum_{k=1}^{p} c_{k}(\boldsymbol{a}) \log \left\{\widehat{f}_{\beta / a_{k}}(x)+\frac{\epsilon}{a_{k}}\right\}\right]=\prod_{k=1}^{p}\left\{\widehat{f}_{\beta / a_{k}}(x)+\frac{\epsilon}{a_{k}}\right\}^{c_{k}(\boldsymbol{a})}, \\
\widehat{f}_{\beta, J F_{\boldsymbol{a}}^{p}}(x) & =\left\{\widehat{f}_{\beta}(x)+\epsilon\right\} \exp \left[\sum_{j=1}^{p-1} \frac{(-1)^{j-1}}{j}\left\{\frac{\widehat{f}_{\beta, A D D_{\boldsymbol{a}}^{p}}(x)}{\widehat{f}_{\beta}(x)+\epsilon}-1\right\}^{j}\right]
\end{aligned}
$$

respectively, where $\epsilon=\epsilon_{\beta} \rightarrow 0$, specified later, is introduced to avoid $\log 0$ and the division by zero, and $\left\{c_{1}(\boldsymbol{a}), \ldots, c_{p}(\boldsymbol{a})\right\}$ is unique solution of

$$
\sum_{k=1}^{p} c_{k}(\boldsymbol{a})=1, \quad \sum_{k=1}^{p} \frac{c_{k}(\boldsymbol{a})}{a_{k}^{\ell}}=0, \quad \ell=1, \ldots, p-1 .
$$

The following result (Lemma 4), independent of interest, enables us to see that

$$
\sum_{k=1}^{p} \frac{c_{k}(\boldsymbol{a})}{a_{k}^{p}}=\frac{(-1)^{p-1}}{\prod_{k=1}^{p} a_{k}}
$$

Lemma 4 For any $\boldsymbol{z}=\left(z_{1}, \ldots, z_{p}\right)^{\prime} \in \mathbb{R}^{p}$, let $\mathcal{V}(\boldsymbol{z})$ be a Vandermonde matrix of $p \times p$, whose $j$ th column is the vector $\left(1, z_{j}, \ldots, z_{j}^{p-1}\right)^{\prime}$ for $j=1, \ldots, p$. If $\mathcal{V}(\boldsymbol{z})$ is invertible (i.e., the $z_{j}$ 's are assumed to be distinct), then, $\prod_{j=1}^{p} z_{j}=(-1)^{p-1} \sum_{j=1}^{p} z_{j}^{p}\left[\mathcal{V}^{-1}(\boldsymbol{z})\right]_{j 1}$, where $\left[\mathcal{V}^{-1}(\boldsymbol{z})\right]_{j k}$ is the $(j, k)$ th element of $\mathcal{V}^{-1}(\boldsymbol{z})$.

Remark 2 The solution $\left\{c_{k}\right\}$ of (7) is computable for user's specified vector $\boldsymbol{a}$, i.e.,

$$
c_{k}(\boldsymbol{a})=\frac{a_{k}^{p-1}}{\prod_{j=1, j \neq k}^{p}\left(a_{k}-a_{j}\right)}, \quad k=1, \ldots, p,
$$

using the inversion of the Vandermonde matrix (e.g., (3.2) of Gautschi (1962)). For example,

- $\boldsymbol{a}=(1,1 / 2, \ldots, 1 / p)^{\prime}$ yields $c_{k}(\boldsymbol{a})=(-1)^{k-1}{ }_{p} C_{k}$ for $k=1, \ldots, p$, and

- $\boldsymbol{a}=(1,(p-1) / p,(p-2) /(p-1), \ldots, 1 / 2)^{\prime}$ (i.e., $a_{1}=1$ and $a_{k}=(p-k+1) /(p-k+2)$ for $k=2, \ldots, p)$ yields $c_{1}(\boldsymbol{a})=p$ ! and $c_{k}(\boldsymbol{a})=(-1)^{k-1}(p-k+1)^{p}{ }_{p} C_{k-2}$ for $k=2, \ldots, p$.

Practically, the selection of $\boldsymbol{a}$ is a difficult problem. In Section 5, numerical studies for $p=2$ and $p=3$ will be conducted by letting $\boldsymbol{a}=(1, a)$ and $\boldsymbol{a}=(1, a, 1 / a)$, respectively, where $a \in(0,1)$. 
Before presenting the main results in this paper, we mention that, as an easy corollary of the strong consistency of the estimator (1), the estimators (4)-(6) are also strong consistent (for the estimators (5) and (6), we additionally assume that $\epsilon \rightarrow 0$ ), i.e., by virtue of Slutsky's lemma, (3) and (7) immediately yield, for fixed $x \in \mathcal{S}$,

- $\widehat{f}_{\beta, A D D_{\boldsymbol{a}}^{p}}(x) \stackrel{a . s .}{\rightarrow} \sum_{k=1}^{p} c_{k}(\boldsymbol{a}) f(x)=f(x)$, and

- if $f(x)>0$, then, $\widehat{f}_{\beta, T S_{\boldsymbol{a}}^{p}}(x) \stackrel{\text { a.s. }}{\rightarrow} \exp \left[\sum_{k=1}^{p} c_{k}(\boldsymbol{a}) \log f(x)\right]=f(x)$ and $\widehat{f}_{\beta, J F_{\boldsymbol{a}}^{p}}(x) \stackrel{\text { a.s. }}{\rightarrow} f(x)$.

\subsection{Asymptotic properties of additive estimator}

To begin with, we consider the additive estimator (4). We write

$$
\begin{aligned}
B_{p, \boldsymbol{a}}(x ; f) & =\frac{(-1)^{p-1}}{\prod_{k=1}^{p} a_{k}} \gamma_{p}(x ; f), \quad \lambda_{p, \boldsymbol{a}}=\sum_{j=1}^{p} \sum_{j^{\prime}=1}^{p} c_{j}(\boldsymbol{a}) c_{j^{\prime}}(\boldsymbol{a})\left(\frac{2 a_{j} a_{j^{\prime}}}{a_{j}+a_{j^{\prime}}}\right)^{1 / 2}, \\
v_{p, \boldsymbol{a}}(\kappa) & =\sum_{j=1}^{p} \sum_{j^{\prime}=1}^{p} c_{j}(\boldsymbol{a}) c_{j^{\prime}}(\boldsymbol{a}) \varsigma_{a_{j}, a_{j^{\prime}}}(\kappa) .
\end{aligned}
$$

Theorem 5 (i) Suppose that Assumptions A4[p] and A5[p](i,ii) hold for some $p \in \mathbb{N} \backslash\{1\}$. Then,

$$
\operatorname{Bias}\left[\widehat{f}_{\beta, A D D_{\boldsymbol{a}}^{p}}(x)\right]=\beta^{p} B_{p, \boldsymbol{a}}(x ; f)+\mathcal{E}_{\beta, A D D_{\boldsymbol{a}}^{p}}(x), \quad x \in \mathcal{S},
$$

where $\mathcal{E}_{\beta, A D D_{a}^{p}}(x)=\sum_{k=1}^{p} c_{k}(\boldsymbol{a}) \mathcal{E}_{\beta / a_{k}, p}(x)$.

(ii) Suppose that Assumptions A1, A2, A4'[1], and A5'(i) hold. Then,

$$
V\left[\widehat{f}_{\beta, A D D_{\boldsymbol{a}}^{p}}(x)\right]= \begin{cases}n^{-1} \beta^{-1 / 2} \lambda_{p, \boldsymbol{a}} V(x ; f)\left[1+O\left(\beta \psi^{-1}(x)\right)\right]+O\left(n^{-1}\right), & x \in \mathcal{S}_{I, \beta}, \\ n^{-1} \beta^{-1} f(x)\left[v_{p, \boldsymbol{a}}(\kappa)+\chi_{\left\{x \notin \mathcal{S}_{B}\right\}} O(1)\right]+O\left(n^{-1}\right), & x \in \mathcal{S}_{B, \beta, \kappa} .\end{cases}
$$

Theorem 6 Suppose that Assumptions A1, A2, A4'[1], and A5'(i) hold. Then,

$$
\begin{array}{cl}
\left(n \beta^{1 / 2}\right)^{1 / 2}\left\{\widehat{f}_{\beta, A D D_{\boldsymbol{a}}^{p}}(x)-E\left[\widehat{f}_{\beta, A D D_{\boldsymbol{a}}^{p}}(x)\right]\right\} \stackrel{d}{\rightarrow} N\left(0, \lambda_{p, \boldsymbol{a}} V(x ; f)\right) & \text { for fixed } x \in \mathcal{S}_{I}, \\
(n \beta)^{1 / 2}\left\{\widehat{f}_{\beta, A D D_{\boldsymbol{a}}^{p}}(x)-E\left[\widehat{f}_{\beta, A D D_{\boldsymbol{a}}^{p}}(x)\right]\right\} \stackrel{d}{\rightarrow} N\left(0, v_{p, \boldsymbol{a}}(0) f(x)\right) & \text { for } x \in \mathcal{S}_{B} .
\end{array}
$$

A replacement of $E\left[\widehat{f}_{\beta, A D D_{\boldsymbol{a}}^{p}}(x)\right]$ by $f(x)$ (or $f(x)+\beta^{p} B_{p, \boldsymbol{a}}(x ; f)$ ) is a routine problem in density estimation theory (use Slutsky's lemma; see Theorems 5(i) and 6) ${ }^{[2]}$.

${ }^{[2]}$ Suppose that Assumptions A1, A2, A4 $[p]$, and A5[p](i,ii) hold for some $p \in \mathbb{N} \backslash\{1\}$. (i). If $n \beta^{(4 p+1) / 2+\eta_{2 p}} \rightarrow 0$, then, for fixed $x \in \mathcal{S}_{I}$,

$$
\left(n \beta^{1 / 2}\right)^{1 / 2}\left\{\widehat{f}_{\beta, A D D_{\boldsymbol{a}}^{p}}(x)-f(x)-\beta^{p} B_{p, \boldsymbol{a}}(x ; f)\right\} \stackrel{d}{\rightarrow} N\left(0, \lambda_{p, \boldsymbol{a}} V(x ; f)\right),
$$

hence, if $n \beta^{(4 p+1) / 2} \rightarrow 0$, then, $\left(n \beta^{1 / 2}\right)^{1 / 2}\left\{\widehat{f}_{\beta, A D D_{\boldsymbol{a}}^{p}}(x)-f(x)\right\} \stackrel{d}{\rightarrow} N\left(0, \lambda_{p, \boldsymbol{a}} V(x ; f)\right)$.

(ii). If $n \beta^{2 p+1+\eta_{2 p}} \rightarrow 0$, then, for $x \in \mathcal{S}_{B}$,

$$
(n \beta)^{1 / 2}\left\{\widehat{f}_{\beta, A D D_{\boldsymbol{a}}^{p}}(x)-f(x)-\beta^{p} B_{p, \boldsymbol{a}}(x ; f)\right\} \stackrel{d}{\rightarrow} N\left(0, v_{p, \boldsymbol{a}}(0) f(x)\right),
$$

hence, if $n \beta^{2 p+1} \rightarrow 0$, then, $(n \beta)^{1 / 2}\left\{\widehat{f}_{\beta, A D D_{\boldsymbol{a}}^{p}}(x)-f(x)\right\} \stackrel{d}{\rightarrow} N\left(0, v_{p, \boldsymbol{a}}(0) f(x)\right)$. 
Theorem 5 shows that

$$
M S E\left[\widehat{f}_{\beta, A D D_{a}^{p}}(x)\right]=\left\{\begin{array}{l}
A M S E\left[\widehat{f}_{\beta, A D D_{a}^{p}}(x)\right]+o\left(\beta^{2 p}+n^{-1} \beta^{-1 / 2}\right) \quad \text { for fixed } x \in \mathcal{S}_{I}, \\
A M S E\left[\widehat{f}_{\beta, A D D_{a}^{p}}(x)\right]+o\left(\beta^{2 p}+n^{-1} \beta^{-1}\right) \quad \text { for } x \in \mathcal{S}_{B, \beta, \kappa},
\end{array}\right.
$$

where

$$
A M S E\left[\widehat{f}_{\beta, A D D_{\boldsymbol{a}}^{p}}(x)\right]=\left\{\begin{array}{l}
\beta^{2 p} B_{p, \boldsymbol{a}}^{2}(x ; f)+n^{-1} \beta^{-1 / 2} \lambda_{p, \boldsymbol{a}} V(x ; f) \text { for fixed } x \in \mathcal{S}_{I}, \\
\beta^{2 p} B_{p, \boldsymbol{a}}^{2}(x ; f)+n^{-1} \beta^{-1} v_{p, \boldsymbol{a}}(\kappa) f(x) \text { for } x \in \mathcal{S}_{B, \beta, \kappa} .
\end{array}\right.
$$

Note that

$\min _{\beta>0} A M S E\left[\widehat{f}_{\beta, A D D_{\boldsymbol{a}}^{p}}(x)\right]= \begin{cases}\frac{4 p+1}{4 p}\left[4 p B_{p, \boldsymbol{a}}^{2}(x ; f)\left\{\lambda_{p, \boldsymbol{a}} V(x ; f) n^{-1}\right\}^{4 p}\right]^{1 /(4 p+1)} & \text { for fixed } x \in \mathcal{S}_{I}, \\ \frac{2 p+1}{2 p}\left[2 p B_{p, \boldsymbol{a}}^{2}(x ; f)\left\{v_{p, \boldsymbol{a}}(\kappa) f(x) n^{-1}\right\}^{2 p}\right]^{1 /(2 p+1)} & \text { for } x \in \mathcal{S}_{B, \beta, \kappa}\end{cases}$

(we assume $\gamma_{p}(x ; f) \neq 0$ ). Although the additive estimator (4) has the slower convergence rate near the boundary $\mathcal{S}_{B}$, such a different rate is asymptotically negligible on the MISE.

Theorem 7 Suppose that Assumptions A1-A3, A4[p], and A5[p] hold for some $p \in \mathbb{N} \backslash\{1\}$. Then,

$$
\operatorname{MISE}\left[\widehat{f}_{\beta, A D D_{a}^{p}}\right]=A M I S E\left[\widehat{f}_{\beta, A D D_{a}^{p}}\right]+o\left(\beta^{2 p}+n^{-1} \beta^{-1 / 2}\right),
$$

where

$$
A M I S E\left[\widehat{f}_{\beta, A D D_{\boldsymbol{a}}^{p}}\right]=\beta^{2 p} \int_{\mathcal{S}} B_{p, \boldsymbol{a}}^{2}(x ; f) d x+n^{-1} \beta^{-1 / 2} \lambda_{p, \boldsymbol{a}} \int_{\mathcal{S}} V(x ; f) d x
$$

is minimized at

$$
\beta=\left[\frac{\lambda_{p, \boldsymbol{a}} \int_{\mathcal{S}} V(x ; f) d x}{4 p \int_{\mathcal{S}} B_{p, \boldsymbol{a}}^{2}(x ; f) d x} n^{-1}\right]^{2 /(4 p+1)}
$$

when $\gamma_{p}(x ; f) \not \equiv 0$, that is,

$$
\min _{\beta>0} A M I S E\left[\widehat{f}_{\beta, A D D_{\boldsymbol{a}}^{p}}\right]=\frac{4 p+1}{4 p}\left[4 p \int_{\mathcal{S}} B_{p, \boldsymbol{a}}^{2}(x ; f) d x\left\{\lambda_{p, \boldsymbol{a}} \int_{\mathcal{S}} V(x ; f) d x n^{-1}\right\}^{4 p}\right]^{1 /(4 p+1)} .
$$

Remark 3 The additive estimator (4) loses the nonnegativity. However, it is easily remedied by considering the positive part $\widehat{f}_{\beta, A D D_{\boldsymbol{a}}^{p}}^{+}(x)=\max \left\{\widehat{f}_{\beta, A D D_{\boldsymbol{a}}^{p}}(x), 0\right\}$. Not surprisingly, $\widehat{f}_{\beta, A D D_{\boldsymbol{a}}^{p}}^{+}(x)$ is superior to $\widehat{f}_{\beta, A D D_{\boldsymbol{a}}^{p}}(x)$ in the (non-asymptotic) sense that, for any $x \in \mathcal{S}$,

$$
\begin{aligned}
& M S E\left[\widehat{f}_{\beta, A D D_{\boldsymbol{a}}^{p}}(x)\right]-M S E\left[\widehat{f}_{\beta, A D D_{\boldsymbol{a}}^{p}}^{+}(x)\right] \\
& =E\left[\widehat{f}_{\beta, A D D_{\boldsymbol{a}}^{p}}^{2}(x) \chi_{\left\{\widehat{f}_{\beta, A D D_{\boldsymbol{a}}^{p}}(x)<0\right\}}\right]-2 f(x) E\left[\widehat{f}_{\beta, A D D_{\boldsymbol{a}}^{p}}(x) \chi_{\left\{\widehat{f}_{\beta, A D D_{\boldsymbol{a}}^{p}}(x)<0\right\}}\right] \geq 0,
\end{aligned}
$$

hence, $\operatorname{MISE}\left[\widehat{f}_{\beta, A D D_{\boldsymbol{a}}^{p}}^{+}\right] \leq \operatorname{MISE}\left[\widehat{f}_{\beta, A D D_{\boldsymbol{a}}^{p}}\right]$. 


\subsection{Asymptotic properties of TS-type and JF-type estimators}

We turn to the TS-type and JF-type estimators (5) and (6).

When $\mathcal{S}=[0, \infty)$, for rigorous asymptotic analyses as in Igarashi and Kakizawa (2018a), we pre-determine, for some constant $\eta \in(0,1]$,

$$
\left(\iota, \iota_{0}\right) \in\{(0,0)\} \bigcup\left\{\left(\iota, \iota_{0}\right) \mid 0<\iota<\frac{\eta / 2}{p+\eta / 2} \text { and } 0<\iota_{0}<\frac{1-(p+1) \iota}{p}\right\}=\widetilde{I}_{p, \eta} \quad(\text { say }),
$$

and consider a set of the points $x$, as follows:

$$
\mathcal{I}_{\iota, \iota_{0}}\left[r_{\beta}\right]=\left\{x \in\left[0, r_{\beta}\right] \mid f(x) \geq \varrho \beta^{\iota_{0}}\right\} \quad \text { with } r_{\beta}=O\left(\beta^{-\iota}\right)
$$

for some $r_{\beta} \equiv r$ (fixed) or $r_{\beta} \rightarrow \infty$ (diverging slowly to infinity), according to $\left(\iota, \iota_{0}\right)=(0,0)$ or $\left(\iota, \iota_{0}\right) \in \widetilde{I}_{p, \eta} \backslash\{(0,0)\}$. Here and subsequently, $\varrho, r>0$ are some constants. Note that, if $\left(\iota, \iota_{0}\right) \in \widetilde{I}_{p, \eta_{2 p}}\left(\subset \widetilde{I}_{p, 1}\right)$ is pre-determined $\left(\eta_{2 p} \in(0,1]\right.$ is given in Assumption A5[p](ii)), then,

$$
r_{\beta}=O\left(\beta^{-\iota}\right) \text { implies } \beta^{\eta_{2 p} / 2}\left(1+r_{\beta}\right)^{p+\eta_{2 p} / 2}+\beta^{1-\iota_{0} p}\left(1+r_{\beta}\right)^{p+1}=o(1) .
$$

For a technical reason, we use the weighted MISE criterion when $\mathcal{S}=[0, \infty)$, i.e.,

$$
\operatorname{MISE}[\widehat{f} ; w]=\int_{0}^{\infty} w(x) M S E[\widehat{f}(x)] d x
$$

where the weight function $w$ is nonnegative, bounded, and continuous except for a finite number of discontinuities (we assume $w(0)>0$ ). On the other hand, when $\mathcal{S}=[0,1]$, unlike the case $\mathcal{S}=[0, \infty)$, no technical difficulty is encountered in approximating the (unweighted) MISE.

In what follows, let $\#=T S, J F$, unless otherwise stated, and let

$$
c_{p, T S}=0 \quad \text { and } \quad c_{p, J F}= \begin{cases}0, & p=2 \text { and } 0<a_{2}<a_{1}=1, \\ 1, & p=2 \text { and } 1=a_{1}<a_{2}, \\ p-1, & p(>2) \text { is even, } \\ p-2, & p(>2) \text { is odd }\end{cases}
$$

We write

$$
B_{\# \boldsymbol{a}}^{p}(x ; f)= \begin{cases}B_{p, \boldsymbol{a}}(x ; f)+\frac{(-1)^{p-1}}{\prod_{k=1}^{p} a_{k}} \sum_{j=2}^{p} \frac{(-1)^{j-1}}{j f^{j-1}(x)} \sum_{\mathcal{L}_{p, j}} \prod_{m=1}^{j} \gamma_{\ell_{m}}(x ; f), & \#=T S, \\ B_{p, \boldsymbol{a}}(x ; f)+\frac{1}{p f^{p-1}(x)} \gamma_{1}^{p}(x ; f), & \#=J F,\end{cases}
$$

where

$$
\mathcal{L}_{p, j}=\left\{\ell_{1}, \ldots, \ell_{j} \in \mathbb{N} \mid \sum_{m=1}^{j} \ell_{m}=p\right\} .
$$

We impose additional assumptions on $\beta, \epsilon, f$, and $w$ :

$\mathrm{A} 6[p]_{\iota_{1}, \iota_{2}} . \beta \propto n^{-\iota_{1}}$ and $\epsilon \propto \beta^{\iota_{2}}$ (independent of $a_{k}, k=1, \ldots, p$ ) for some constants $\iota_{1}$ and $\iota_{2}$. 
A7 $[p]_{\iota_{0}, \iota_{2}}^{\#}$. When $\mathcal{S}=[0, \infty)$, given $r_{\beta} \equiv r$ or $r_{\beta} \rightarrow \infty, f$ satisfies (i) $\min _{x \in\left[0, r_{\beta}\right]} f(x) \geq \varrho \beta^{\iota_{0}}$, and $w$ is a weight function, independent of $\beta$, such that (ii) $\int_{r_{\beta}}^{\infty} w(x) d x \propto \exp \left(-\beta^{-A}\right)$ for some constant $A>c_{p, \#}\left(1+\iota_{2}\right)$, independent of $\beta$, and that (iii) $w(x) B_{\# \boldsymbol{a}}^{2}(x ; f)$ is integrable, where $\iota_{0}$ and $\iota_{2}$ are some constants (when $r_{\beta} \equiv r$, the requirement (ii) holds iff $w$ is a truncated weight function, with $w(y)=0$ for any $y>r)$.

A $7^{\prime}$. When $\mathcal{S}=[0,1], f$ satisfies $\min _{x \in[0,1]} f(x)>0$.

Below, given $p \in \mathbb{N} \backslash\{1\}$ and $\left(\iota, \iota_{0}\right) \in \widetilde{I}_{p, \eta_{2 p}}\left(\eta_{2 p} \in(0,1]\right.$ is given in Assumption A5[p](ii)), we technically take $\left(\iota_{1}, \iota_{2}\right) \in I_{p,\left(\iota, \iota_{0}\right), \# \text {, where }}$

$$
I_{p,\left(\iota, \iota_{0}\right), \#}=\left\{\left(\iota_{1}, \iota_{2}\right) \mid 0<\iota_{1}<\frac{1}{1+2 \iota_{0}+c_{p, \#}\left(1+\iota_{2}\right)}, \quad \iota_{2}>1+\left(\iota+\iota_{0}\right)(p-1)\right\} .
$$

Remark 4 Note that $\beta \propto n^{-1 /(2 p+1 / d)}$ for $d=1,2$ (i.e., $\iota_{1}=2 /(4 p+1)$ and $\iota_{1}=1 /(2 p+1)$ ) are feasible for $\#=T S$ (the same remains valid for $\#=J F$ when $p=2$ and $0<a_{2}<a_{1}=1$ ). In fact, with $c_{p, \#}=0, \iota_{0} \in[0,1 / 2)$ (see (9)) implies $1 /(2 p+1 / 2)<1 / 2<1 /\left(1+2 \iota_{0}\right)$. On the other hand, for the JF-type (with $c_{p, J F}>0$ ), as long as $\left(\iota, \iota_{0}\right) \in \widetilde{I}_{p, \eta_{2 p}}$ satisfies

$$
\iota_{0}<\frac{2 p-1 / 2-2 c_{p, J F}-c_{p, J F}(p-1) \iota}{c_{p, J F}(p-1)+2}
$$

we see that

$$
1+\left(\iota+\iota_{0}\right)(p-1)<\iota_{2}<\frac{2 p-1 / 2-c_{p, J F}-2 \iota_{0}}{c_{p, J F}}
$$

implies that $1 /(2 p+1 / 2)<1 /\left\{1+2 \iota_{0}+c_{p, J F}\left(1+\iota_{2}\right)\right\}$. Hence, to ensure the feasibility of $\beta \propto n^{-1 /(2 p+1 / d)}$ for $d=1,2$ and $\#=T S, J F$, we take $\left(\iota, \iota_{0}\right) \in \widetilde{I}_{p, \eta_{2 p}, \#}\left(\subset \widetilde{I}_{p, \eta_{2 p}}\right)$, where

$$
\widetilde{I}_{p, \eta_{2 p}, \#}= \begin{cases}\widetilde{I}_{p, \eta_{2 p}}, & c_{p, \#}=0 \\ \widetilde{I}_{p, \eta_{2 p}} \bigcap\left\{\left(\iota, \iota_{0}\right) \mid \iota_{0}<\frac{2 p-1 / 2-2 c_{p, \#}-c_{p, \#}(p-1) \iota}{c_{p, \#}(p-1)+2}\right\}, & c_{p, \#}>0\end{cases}
$$

\subsection{1. $\quad$ Case $\mathcal{S}=[0, \infty)$}

We are ready to present the asymptotic properties of the TS-type and JF-type estimators (5) and (6) for the case $\mathcal{S}=[0, \infty)$.

Theorem 8 (i) Given $p \in \mathbb{N} \backslash\{1\}$, suppose that Assumptions A1(i), A4[p], and A5[p](i,ii) hold. In addition, given $\left(\iota, \iota_{0}\right) \in \widetilde{I}_{p, \eta_{2 p}}\left(\subset \widetilde{I}_{p, 1}\right)$, where $\eta_{2 p} \in(0,1]$ is given in Assumption $\mathrm{A} 5[p](\mathrm{ii})$,

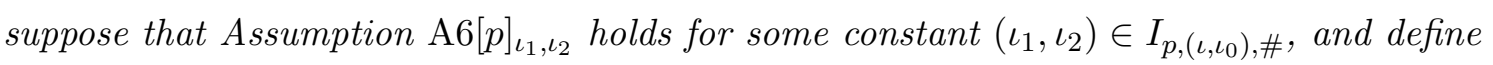

$$
\omega_{\beta}(x)=\beta^{\eta_{2 p} / 2}(1+x)^{p+\eta_{2 p} / 2}+\beta^{1-\iota_{0} p}(1+x)^{p+1}+\beta^{\iota_{2}-1-\iota_{0}(p-1)}(1+x)^{p-1} .
$$

Then,

$$
\operatorname{Bias}\left[\widehat{f}_{\beta, \#_{\boldsymbol{a}}^{p}}(x)\right]=\beta^{p} B_{\#_{\boldsymbol{a}}^{p}}(x ; f)+\mathcal{E}_{\beta, \#_{\boldsymbol{a}}^{p}}(x) \quad \text { for } x \in \mathcal{I}_{\iota, \iota_{0}}\left[r_{\beta}\right],
$$


where

$$
\mathcal{E}_{\beta, \# \boldsymbol{a}}^{p}(x)=O\left(\beta^{p} \omega_{\beta}(x)+\beta^{-\iota_{0}} \sum_{k=1}^{p} V\left[\widehat{f}_{\beta / a_{k}}(x)\right]\right) .
$$

(ii) Given $p \in \mathbb{N} \backslash\{1\}$ and $\left(\iota, \iota_{0}\right) \in \widetilde{I}_{p, 1}$, suppose that Assumptions A1, A2, A4' $[1]$, A5'(i), and

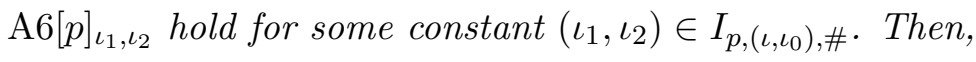

$$
V\left[\widehat{f}_{\beta, \# \boldsymbol{a}}^{p}(x)\right]=V\left[\widehat{f}_{\beta, A D D_{\boldsymbol{a}}^{p}}(x)\right]+\widetilde{\mathcal{E}}_{\beta, \#_{\boldsymbol{a}}^{p}}(x) \quad \text { for } x \in \mathcal{I}_{\iota, \iota_{0}}\left[r_{\beta}\right]
$$

where

$$
\widetilde{\mathcal{E}}_{\beta, \# \boldsymbol{a}}^{p}(x)=O\left(\beta^{2 p+1-\iota_{0} p}(1+x)^{p+1}+\left\{\beta^{1-\iota_{0} p}(1+x)^{p+1}+n^{-1 / 2} \beta^{-\left(1 / 2+\iota_{0}\right)}\right\} \sum_{k=1}^{p} V\left[\widehat{f}_{\beta / a_{k}}(x)\right]\right) .
$$

Theorem 9 Given $p \in \mathbb{N} \backslash\{1\}$, suppose that Assumptions A1, A2, A4 $[1]$, A5'(i), and A6 $[p]_{\iota_{1}, \iota_{2}}$ hold for some constant $\left(\iota_{1}, \iota_{2}\right) \in I_{p, 2, \#}$ for $x \in \mathcal{S}_{I}$ or $\left(\iota_{1}, \iota_{2}\right) \in I_{p, 1, \#}$ for $x \in \mathcal{S}_{B}$ (note that $I_{p, 2, \#} \subset I_{p, 1, \#} \subset I_{p,(0,0), \#) \text {, where }}$

$$
I_{p, d, \#}=\left\{\left(\iota_{1}, \iota_{2}\right) \mid \frac{1}{2 p+2+1 / d}<\iota_{1}<\frac{1}{1+c_{p, \#}\left(1+\iota_{2}\right)}, \quad 1<\iota_{2}<\frac{2 p+1+1 / d-c_{p, \#}}{c_{p, \#}}\right\}
$$

(exceptionally, when $c_{p, \#}=0$, the feasible range of $\iota_{2}$ should read as " $\iota_{2}>1$ "). Then,

$$
\begin{aligned}
& \left(n \beta^{1 / 2}\right)^{1 / 2}\left\{\widehat{f}_{\beta, \#_{\boldsymbol{a}}^{p}}(x)-E\left[\widehat{f}_{\beta, \#_{\boldsymbol{a}}^{p}}(x)\right]\right\} \stackrel{d}{\rightarrow} N\left(0, \lambda_{p, \boldsymbol{a}} V(x ; f)\right) \quad \text { for fixed } x \in \mathcal{I}_{0,0}[r] \cap \mathcal{S}_{I}, \\
& (n \beta)^{1 / 2}\left\{\widehat{f}_{\beta, \#_{\boldsymbol{a}}^{p}}(x)-E\left[\widehat{f}_{\beta, \#_{\boldsymbol{a}}^{p}}(x)\right]\right\} \stackrel{d}{\rightarrow} N\left(0, v_{p, \boldsymbol{a}}(0) f(x)\right) \quad \text { for } x \in \mathcal{I}_{0,0}[r] \cap \mathcal{S}_{B} .
\end{aligned}
$$

A replacement of $E\left[\widehat{f}_{\beta, \#_{\boldsymbol{a}}^{p}}(x)\right]$ by $f(x)$ (or $\left.f(x)+\beta^{p} B_{\#_{\boldsymbol{a}}^{p}}(x ; f)\right)$ is a routine problem in density estimation theory (use Slutsky's lemma; see Theorems 8(i) and 9) ${ }^{[3]}$.

Theorem 8, together with Theorem 5(ii), shows that

$$
M S E\left[\widehat{f}_{\beta, \#_{a}^{p}}(x)\right]=\left\{\begin{array}{l}
A M S E\left[\widehat{f}_{\beta, \#_{a}^{p}}(x)\right]+o\left(\beta^{2 p}+n^{-1} \beta^{-1 / 2}\right) \quad \text { for fixed } x \in \mathcal{I}_{0,0}[r] \cap \mathcal{S}_{I}, \\
A M S E\left[\widehat{f}_{\beta, \#_{a}^{p}}^{p}(x)\right]+o\left(\beta^{2 p}+n^{-1} \beta^{-1}\right) \text { for } x \in \mathcal{I}_{0,0}[r] \cap \mathcal{S}_{B, \beta, \kappa},
\end{array}\right.
$$

where

$$
A M S E\left[\widehat{f}_{\beta, \#_{\boldsymbol{a}}^{p}}(x)\right]=\left\{\begin{array}{l}
\beta^{2 p} B_{\#_{\boldsymbol{a}}^{p}}^{2}(x ; f)+n^{-1} \beta^{-1 / 2} \lambda_{p, \boldsymbol{a}} V(x ; f) \text { for fixed } x \in \mathcal{I}_{0,0}[r] \cap \mathcal{S}_{I}, \\
\beta^{2 p} B_{\#_{\boldsymbol{a}}^{p}}^{2}(x ; f)+n^{-1} \beta^{-1} v_{p, \boldsymbol{a}}(\kappa) f(x) \text { for } x \in \mathcal{I}_{0,0}[r] \cap \mathcal{S}_{B, \beta, \kappa} .
\end{array}\right.
$$

${ }^{[3]}$ Given $p \in \mathbb{N} \backslash\{1\}$, suppose that Assumptions A1, A2, A4 $[p], \mathrm{A} 5[p](\mathrm{i}, \mathrm{ii})$, and A6[p] $]_{\iota_{1}, \iota_{2}}$ hold for some constant

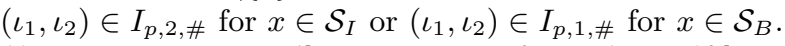

(i). If, in addition, $2 /\left[4 p+1+2 \min \left\{\eta_{2 p}, 2\left(\iota_{2}-1\right)\right\}\right]<\iota_{1}$, then, for fixed $x \in \mathcal{I}_{0,0}[r] \cap \mathcal{S}_{I}$,

$$
\left(n \beta^{1 / 2}\right)^{1 / 2}\left\{\widehat{f}_{\beta, \#_{a}^{p}}(x)-f(x)-\beta^{p} B_{\#_{a}^{p}}(x ; f)\right\} \stackrel{d}{\rightarrow} N\left(0, \lambda_{p, a} V(x ; f)\right),
$$

hence, if, in addition, $2 /(4 p+1)<\iota_{1}$, then, $\left(n \beta^{1 / 2}\right)^{1 / 2}\left\{\widehat{f}_{\beta, \#_{a}^{p}}(x)-f(x)\right\} \stackrel{d}{\rightarrow} N\left(0, \lambda_{p, \boldsymbol{a}} V(x ; f)\right)$.

(ii). If, in addition, $1 /\left[2 p+1+\min \left\{\eta_{2 p}, 2\left(\iota_{2}-1\right)\right\}\right]<\iota_{1}$, then, for $x \in \mathcal{I}_{0,0}[r] \cap \mathcal{S}_{B}$,

$$
(n \beta)^{1 / 2}\left\{\widehat{f}_{\beta, \#_{a}^{p}}(x)-f(x)-\beta^{p} B_{\#_{a}^{p}}(x ; f)\right\} \stackrel{d}{\rightarrow} N\left(0, v_{p, \boldsymbol{a}}(0) f(x)\right),
$$

hence, if, in addition, $1 /(2 p+1)<\iota_{1}$, then, $(n \beta)^{1 / 2}\left\{\widehat{f}_{\beta, \#_{\boldsymbol{a}}^{p}}(x)-f(x)\right\} \stackrel{d}{\rightarrow} N\left(0, v_{p, \boldsymbol{a}}(0) f(x)\right)$. 
Note that

$$
\begin{aligned}
& \min _{\beta>0} A M S E\left[\widehat{f}_{\beta, \#_{\boldsymbol{a}}^{p}}(x)\right] \\
& = \begin{cases}\frac{4 p+1}{4 p}\left[4 p B_{\#_{\boldsymbol{a}}^{p}}^{2}(x ; f)\left\{\lambda_{p, \boldsymbol{a}} V(x ; f) n^{-1}\right\}^{4 p}\right]^{1 /(4 p+1)} & \text { for fixed } x \in \mathcal{I}_{0,0}[r] \cap \mathcal{S}_{I}, \\
\frac{2 p+1}{2 p}\left[2 p B_{\#_{\boldsymbol{a}}^{p}}^{2}(x ; f)\left\{v_{p, \boldsymbol{a}}(\kappa) f(x) n^{-1}\right\}^{2 p}\right]^{1 /(2 p+1)} & \text { for } x \in \mathcal{I}_{0,0}[r] \cap \mathcal{S}_{B, \beta, \kappa}\end{cases}
\end{aligned}
$$

(see Remark 4, with $\left.\left(\iota, \iota_{0}\right)=(0,0)\right)$, provided that $B_{\#_{a}^{p}}(x ; f) \neq 0$. Although the TS/JF-type estimators (5) and (6) have the slower convergence rate near the boundary $\mathcal{S}_{B}$, such a different rate is asymptotically negligible on the weighted MISE.

Theorem 10 Given $p \in \mathbb{N} \backslash\{1\}$, suppose that Assumptions A1-A3, A4[p], and A5[p] hold. In addition, given $\left(\iota, \iota_{0}\right) \in \widetilde{I}_{p, \eta_{2 p}}\left(\eta_{2 p} \in(0,1]\right.$ is given in Assumption $\left.\mathrm{A} 5[p](\mathrm{ii})\right)$, suppose that

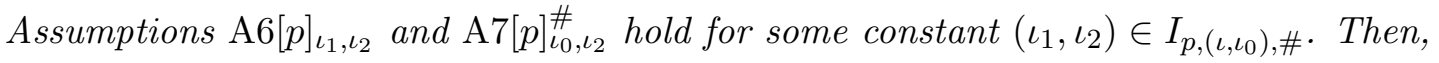

$$
\operatorname{MISE}\left[\widehat{f}_{\beta, \#_{a}^{p}} ; w\right]=\operatorname{AMISE}\left[\widehat{f}_{\beta, \#_{a}^{p}} ; w\right]+o\left(\beta^{2 p}+n^{-1} \beta^{-1 / 2}\right),
$$

where

$$
A M I S E\left[\widehat{f}_{\beta, \#_{\boldsymbol{a}}^{p}} ; w\right]=\beta^{2 p} \int_{0}^{\infty} w(x) B_{\#_{\boldsymbol{a}}^{p}}^{2}(x ; f) d x+n^{-1} \beta^{-1 / 2} \lambda_{p, \boldsymbol{a}} \int_{0}^{\infty} w(x) V(x ; f) d x
$$

is minimized at

$$
\beta=\left[\frac{\lambda_{p, \boldsymbol{a}} \int_{0}^{\infty} w(x) V(x ; f) d x}{4 p \int_{0}^{\infty} w(x) B_{\#_{\boldsymbol{a}}^{p}}^{2}(x ; f) d x} n^{-1}\right]^{2 /(4 p+1)}
$$

(it is feasible for $\left(\iota, \iota_{0}\right) \in \widetilde{I}_{p, \eta_{2 p}, \#}\left(\subset \widetilde{I}_{p, \eta_{2 p}}\right)$; see Remark 4$)$ when $\sqrt{w(x)} B_{\# p}^{p}(x ; f) \not \equiv 0$, that is,

$$
\begin{aligned}
& \min _{\beta>0} \operatorname{AMISE}\left[\widehat{f}_{\beta, \#_{\boldsymbol{a}}^{p}} ; w\right] \\
& =\frac{4 p+1}{4 p}\left[4 p \int_{0}^{\infty} w(x) B_{\#_{\boldsymbol{a}}^{p}}^{2}(x ; f) d x\left\{\lambda_{p, \boldsymbol{a}} \int_{0}^{\infty} w(x) V(x ; f) d x n^{-1}\right\}^{4 p}\right]^{1 /(4 p+1)} .
\end{aligned}
$$

Remark 5 If possible, it will be better for us not to use the weighted MISE criterion. However, at present, we do not yet realize whether or not the valid asymptotic expansion

$$
M I S E\left[\widehat{f}_{\beta, \#_{\boldsymbol{a}}^{p}}\right]=\beta^{2 p} \int_{0}^{\infty} B_{\#_{\boldsymbol{a}}^{p}}^{2}(x ; f) d x+n^{-1} \beta^{-1 / 2} \lambda_{p, \boldsymbol{a}} \int_{0}^{\infty} V(x ; f) d x+o\left(\beta^{2 p}+n^{-1} \beta^{-1 / 2}\right)
$$

can be obtained for the case $w(x) \equiv 1$.

Here are some examples of $(w, f)$ that we can apply Theorem 10.

(a) For a truncated weight function $w$, with $w(y)=0$ for any $y>r$, Theorem 10 is applicable, whenever $\min _{x \in[0, r]} f(x)>0\left(\right.$ choose $\left(\iota, \iota_{0}\right)=(0,0)$ and $\left.r_{\beta} \equiv r\right)$.

(b) Let $w^{\dagger}(x) \propto x^{c_{0}-1} \exp \left\{x^{c_{0}}-\exp \left(x^{c_{0}}\right)\right\}$ (say) for some constant $c_{0}>1$. Suppose that $w(x) \leq w^{\dagger}(x)$ for all sufficiently large $x$, and that there exists a constant $c_{1}>0$ such that 
$\min _{x \geq 0}\left\{f(x) \exp \left(c_{1} x\right)\right\}>0$ (in this case, $w(x) B_{\#_{a}^{p}}^{2}(x ; f)$ is integrable). Then, given $p \in \mathbb{N} \backslash\{1\}$ and the pair $\left(\iota, \iota_{0}\right) \in \widetilde{I}_{p, \eta_{2 p}} \backslash\{(0,0)\} \quad\left(\eta_{2 p} \in(0,1]\right.$ is given in Assumption A5[p](ii)), we choose $r_{\beta}=\left(\iota_{0} / c_{1}\right) \log (1 / \beta)$ to verify that

- $\min _{x \in\left[0, r_{\beta}\right]} f(x) \geq \varrho \beta^{\iota_{0}}$, where $\varrho=\min _{x \geq 0}\left\{f(x) \exp \left(c_{1} x\right)\right\}$,

- $\int_{r_{\beta}}^{\infty} x^{c_{0}-1} \exp \left\{x^{c_{0}}-\exp \left(x^{c_{0}}\right)\right\} d x=\exp \left(-\beta^{-\left(\iota_{0} / c_{1}\right)^{c_{0}}\{\log (1 / \beta)\}^{c_{0}-1}}\right)$, where, for any constant $A>0$ and all sufficiently small $\beta>0,\left(\iota_{0} / c_{1}\right)^{c_{0}}\{\log (1 / \beta)\}^{c_{0}-1}>A$.

(c) Let $w^{\dagger}(x) \propto e^{-x}$ or $w^{\dagger}(x) \propto \exp \{x-\exp (x)\}$ (say) according to \# $=T S$ or $\#=J F$. Suppose that $w(x) \leq w^{\dagger}(x)$ for all sufficiently large $x$, and that there exists a constant $c_{1}>1$ such that $\min _{x \geq 0}\left\{f(x)(1+x)^{c_{1}}\right\}>0$ (in this case, $w(x) B_{\#_{\boldsymbol{a}}^{p}}^{2}(x ; f)$ is integrable). We choose $r_{\beta}=\beta^{-\iota_{0} / c_{1}}-1$ $\left(=O\left(\beta^{-\iota}\right)\right)$, where, given $p \in \mathbb{N} \backslash\{1\}$, the pair $\left(\iota, \iota_{0}\right) \in \widetilde{I}_{p, \eta_{2 p}} \backslash\{(0,0)\} \quad\left(\eta_{2 p} \in(0,1]\right.$ is given in Assumption A5[p](ii)) is pre-determined according to the inequalities $0<\iota<\eta_{2 p} /\left(2 p+\eta_{2 p}\right)$, $0<\iota_{0}<\{1-(p+1) \iota\} / p$, and $\iota_{0} \leq c_{1} \iota$; more precisely,

- if $\eta_{2 p} \in\left(0,2 /\left(1+c_{1}\right)\right]$, then, $\left(\iota, \iota_{0}\right) \in \widetilde{I}_{p, \eta_{2 p}}^{[1]} \subset \widetilde{I}_{p, \eta_{2 p}} \backslash\{(0,0)\}$, where

$$
\widetilde{I}_{p, \eta_{2 p}}^{[1]}=\left\{\left(\iota, \iota_{0}\right) \mid 0<\iota<\frac{\eta_{2 p} / 2}{p+\eta_{2 p} / 2} \text { and } 0<\iota_{0} \leq c_{1} \iota\right\}
$$

- if $\eta_{2 p} \in\left(2 /\left(1+c_{1}\right), 1\right]$, then, $\left(\iota, \iota_{0}\right) \in \widetilde{I}_{p}^{[2]} \cup \widetilde{I}_{p, \eta_{2 p}}^{[3]} \subset \widetilde{I}_{p, \eta_{2 p}} \backslash\{(0,0)\}$, where

$$
\begin{aligned}
\widetilde{I}_{p}^{[2]} & =\left\{\left(\iota, \iota_{0}\right) \mid 0<\iota<\frac{1}{\left(1+c_{1}\right) p+1} \text { and } 0<\iota_{0} \leq c_{1} \iota\right\} \\
\widetilde{I}_{p, \eta_{2 p}}^{[3]} & =\left\{\left(\iota, \iota_{0}\right) \mid \frac{1}{\left(1+c_{1}\right) p+1} \leq \iota<\frac{\eta_{2 p} / 2}{p+\eta_{2 p} / 2} \text { and } 0<\iota_{0}<\frac{1-(p+1) \iota}{p}\right\} .
\end{aligned}
$$

Then, we can verify that

- $\min _{x \in\left[0, r_{\beta}\right]} f(x) \geq \varrho \beta^{\iota_{0}}$, where $\varrho=\min _{x \geq 0}\left\{f(x)(1+x)^{c_{1}}\right\}$,

- $\int_{r_{\beta}}^{\infty} e^{-x} d x=\exp \left(-\beta^{-\iota_{0} / c_{1}}+1\right)$,

- $\int_{r_{\beta}}^{\infty} \exp \{x-\exp (x)\} d x=\exp \left\{-\exp \left(\beta^{-\iota_{0} / c_{1}}-1\right)\right\}$, where, for any constant $A>0$ and all sufficiently small $\beta>0, \beta^{-\iota_{0} / c_{1}}-1+A \log \beta>0$.

\subsection{2. $\quad$ Case $\mathcal{S}=[0,1]$}

In line with Igarashi (2016a), let $\mathcal{I}=\{x \in[0,1] \mid f(x) \geq \varrho\}$ (note $\mathcal{I}=\mathcal{I}_{0,0}[1]$ ).

The following results for the case $\mathcal{S}=[0,1]$ are counterparts of Theorems 8-10 (here, we can handle the (unweighted) MISE without any difficulty). 
Theorem $8^{\prime}$ (i) Given $p \in \mathbb{N} \backslash\{1\}$, suppose that Assumptions A1(i), A4[p], A5[p](i,ii), and

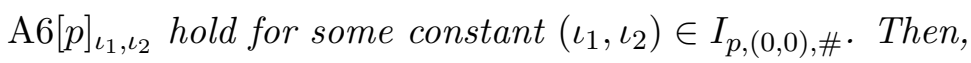

$$
\operatorname{Bias}\left[\widehat{f}_{\beta, \#_{\boldsymbol{a}}^{p}}(x)\right]=\beta^{p} B_{\#_{\boldsymbol{a}}^{p}}(x ; f)+\mathcal{E}_{\beta, \#_{\boldsymbol{a}}^{p}}(x) \quad \text { for } x \in \mathcal{I},
$$

where

$$
\mathcal{E}_{\beta, \# \boldsymbol{a}}^{p}(x)=O\left(\beta^{p+\min \left(\eta_{2 p} / 2, \iota_{2}-1\right)}+\sum_{k=1}^{p} V\left[\widehat{f}_{\beta / a_{k}}(x)\right]\right) .
$$

(ii) Given $p \in \mathbb{N} \backslash\{1\}$, suppose that Assumptions A1, A2, A4 $4^{\prime}[1]$, A5'(i), and $\mathrm{A} 6[p]_{\iota_{1}, \iota_{2}}$ hold for

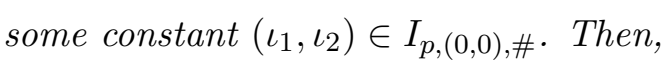

$$
V\left[\widehat{f}_{\beta, \# \boldsymbol{a}}^{p}(x)\right]=V\left[\widehat{f}_{\beta, A D D_{\boldsymbol{a}}^{p}}(x)\right]+\widetilde{\mathcal{E}}_{\beta, \#_{\boldsymbol{a}}^{p}}(x) \quad \text { for } x \in \mathcal{I},
$$

where

$$
\widetilde{\mathcal{E}}_{\beta, \# \boldsymbol{a}}(x)=O\left(\beta^{2 p+1}+\left(\beta+n^{-1 / 2} \beta^{-1 / 2}\right) \sum_{k=1}^{p} V\left[\widehat{f}_{\beta / a_{k}}(x)\right]\right) .
$$

Theorem 9' Given $p \in \mathbb{N} \backslash\{1\}$, suppose that Assumptions A1, A2, A4' $[1], \mathrm{A} 5^{\prime}(\mathrm{i})$, and $\mathrm{A} 6[p]_{\iota_{1}, \iota_{2}}$ hold for some constant $\left(\iota_{1}, \iota_{2}\right) \in I_{p, 2, \#}$ for $x \in \mathcal{S}_{I}$ or $\left(\iota_{1}, \iota_{2}\right) \in I_{p, 1, \#}$ for $x \in \mathcal{S}_{B}$ (note that $I_{p, 2, \#} \subset I_{p, 1, \#} \subset I_{p,(0,0), \#)}$. Then,

$$
\begin{aligned}
& \left(n \beta^{1 / 2}\right)^{1 / 2}\left\{\widehat{f}_{\beta, \#_{\boldsymbol{a}}^{p}}(x)-E\left[\widehat{f}_{\beta, \#_{\boldsymbol{a}}^{p}}(x)\right]\right\} \stackrel{d}{\rightarrow} N\left(0, \lambda_{p, \boldsymbol{a}} V(x ; f)\right) \quad \text { for fixed } x \in \mathcal{I} \cap \mathcal{S}_{I}, \\
& (n \beta)^{1 / 2}\left\{\widehat{f}_{\beta, \#_{\boldsymbol{a}}^{p}}(x)-E\left[\widehat{f}_{\beta, \#_{\boldsymbol{a}}^{p}}(x)\right]\right\} \stackrel{d}{\rightarrow} N\left(0, v_{p, \boldsymbol{a}}(0) f(x)\right) \quad \text { for } x \in \mathcal{I} \cap \mathcal{S}_{B} .
\end{aligned}
$$

A replacement of $E\left[\widehat{f}_{\beta, \#_{\boldsymbol{a}}^{p}}(x)\right]$ by $f(x)$ (or $f(x)+\beta^{p} B_{\#_{\boldsymbol{a}}^{p}}(x ; f)$ ) is a routine problem in density estimation theory (use Slutsky's lemma; see Theorems $8^{\prime}(\mathrm{i})$ and $\left.9^{\prime}\right)^{[4]}$.

Theorem $8^{\prime}$, together with Theorem 5(ii), shows that

$$
M S E\left[\widehat{f}_{\beta, \#_{a}^{p}}(x)\right]=\left\{\begin{array}{l}
A M S E\left[\widehat{f}_{\beta}, \#_{a}^{p}(x)\right]+o\left(\beta^{2 p}+n^{-1} \beta^{-1 / 2}\right) \quad \text { for fixed } x \in \mathcal{I} \cap \mathcal{S}_{I}, \\
A M S E\left[\widehat{f}_{\beta, \#_{a}^{p}}(x)\right]+o\left(\beta^{2 p}+n^{-1} \beta^{-1}\right) \text { for } x \in \mathcal{I} \cap \mathcal{S}_{B, \beta, \kappa},
\end{array}\right.
$$

where

$$
A M S E\left[\widehat{f}_{\beta, \#_{\boldsymbol{a}}^{p}}(x)\right]=\left\{\begin{array}{l}
\beta^{2 p} B_{\#_{\boldsymbol{a}}^{p}}^{2}(x ; f)+n^{-1} \beta^{-1 / 2} \lambda_{p, \boldsymbol{a}} V(x ; f) \quad \text { for fixed } x \in \mathcal{I} \cap \mathcal{S}_{I}, \\
\beta^{2 p} B_{\#_{\boldsymbol{a}}^{p}}^{2}(x ; f)+n^{-1} \beta^{-1} v_{p, \boldsymbol{a}}(\kappa) f(x) \text { for } x \in \mathcal{I} \cap \mathcal{S}_{B, \beta, \kappa} .
\end{array}\right.
$$

${ }^{[4]}$ Given $p \in \mathbb{N} \backslash\{1\}$, suppose that Assumptions A1, A2, A4[p], A5[p](i,ii), and A6[p] $]_{\iota_{1}, \iota_{2}}$ hold for some constant

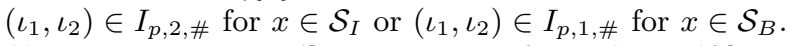

(i). If, in addition, $2 /\left[4 p+1+2 \min \left\{\eta_{2 p}, 2\left(\iota_{2}-1\right)\right\}\right]<\iota_{1}$, then, for fixed $x \in \mathcal{I} \cap \mathcal{S}_{I}$,

$$
\left(n \beta^{1 / 2}\right)^{1 / 2}\left\{\widehat{f}_{\beta, \#_{\boldsymbol{a}}^{p}}(x)-f(x)-\beta^{p} B_{\#_{\boldsymbol{a}}^{p}}(x ; f)\right\} \stackrel{d}{\rightarrow} N\left(0, \lambda_{p, \boldsymbol{a}} V(x ; f)\right),
$$

hence, if, in addition, $2 /(4 p+1)<\iota_{1}$, then, $\left(n \beta^{1 / 2}\right)^{1 / 2}\left\{\widehat{f}_{\beta, \#_{a}^{p}}(x)-f(x)\right\} \stackrel{d}{\rightarrow} N\left(0, \lambda_{p, \boldsymbol{a}} V(x ; f)\right)$.

(ii). If, in addition, $1 /\left[2 p+1+\min \left\{\eta_{2 p}, 2\left(\iota_{2}-1\right)\right\}\right]<\iota_{1}$, then, for $x \in \mathcal{I} \cap \mathcal{S}_{B}$,

$$
(n \beta)^{1 / 2}\left\{\widehat{f}_{\beta, \#_{a}^{p}}(x)-f(x)-\beta^{p} B_{\#_{a}^{p}}(x ; f)\right\} \stackrel{d}{\rightarrow} N\left(0, v_{p, \boldsymbol{a}}(0) f(x)\right),
$$

hence, if, in addition, $1 /(2 p+1)<\iota_{1}$, then, $(n \beta)^{1 / 2}\left\{\widehat{f}_{\beta, \#_{\boldsymbol{a}}^{p}}(x)-f(x)\right\} \stackrel{d}{\rightarrow} N\left(0, v_{p, \boldsymbol{a}}(0) f(x)\right)$. 
Note that

$\min _{\beta>0} A M S E\left[\widehat{f}_{\beta, \#_{\boldsymbol{a}}^{p}}(x)\right]= \begin{cases}\frac{4 p+1}{4 p}\left[4 p B_{\#_{\boldsymbol{a}}^{p}}^{2}(x ; f)\left\{\lambda_{p, \boldsymbol{a}} V(x ; f) n^{-1}\right\}^{4 p}\right]^{1 /(4 p+1)} & \text { for fixed } x \in \mathcal{I} \cap \mathcal{S}_{I}, \\ \frac{2 p+1}{2 p}\left[2 p B_{\#_{\boldsymbol{a}}^{p}}^{2}(x ; f)\left\{v_{p, \boldsymbol{a}}(\kappa) f(x) n^{-1}\right\}^{2 p}\right]^{1 /(2 p+1)} & \text { for } x \in \mathcal{I} \cap \mathcal{S}_{B, \beta, \kappa}\end{cases}$

(see Remark 4, with $\left.\left(\iota, \iota_{0}\right)=(0,0)\right)$, provided that $B_{\#_{a}^{p}}(x ; f) \neq 0$. Although the TS/JF-type estimators (5) and (6) have the slower convergence rate near the boundary $\mathcal{S}_{B}$, such a different rate is asymptotically negligible on the MISE.

Theorem 10' Given $p \in \mathbb{N} \backslash\{1\}$, suppose that Assumptions A1, A2, A4[p], A5[p](i,ii), A6 $[p]_{\iota_{1}, \iota_{2}}$,

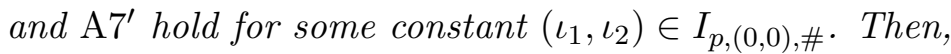

$$
\operatorname{MISE}\left[\widehat{f}_{\beta, \#_{\boldsymbol{a}}^{p}}\right]=A M I S E\left[\widehat{f}_{\beta, \#_{\boldsymbol{a}}^{p}}\right]+o\left(\beta^{2 p}+n^{-1} \beta^{-1 / 2}\right),
$$

where

$$
A M I S E\left[\widehat{f}_{\beta, \#_{\boldsymbol{a}}^{p}}\right]=\beta^{2 p} \int_{0}^{1} B_{\#_{\boldsymbol{a}}^{p}}^{2}(x ; f) d x+n^{-1} \beta^{-1 / 2} \lambda_{p, \boldsymbol{a}} \int_{0}^{1} V(x ; f) d x
$$

is minimized at

$$
\beta=\left[\frac{\lambda_{p, \boldsymbol{a}} \int_{0}^{1} V(x ; f) d x}{4 p \int_{0}^{1} B_{\#_{\boldsymbol{a}}^{p}}^{2}(x ; f) d x} n^{-1}\right]^{2 /(4 p+1)}
$$

(it is feasible; see Remark 4 , with $\left.\left(\iota, \iota_{0}\right)=(0,0)\right)$, when $B_{\#_{a}^{p}}(x ; f) \not \equiv 0$, that is,

$$
\min _{\beta>0} A M I S E\left[\widehat{f}_{\beta, \#_{\boldsymbol{a}}^{p}}\right]=\frac{4 p+1}{4 p}\left[4 p \int_{0}^{1} B_{\#_{\boldsymbol{a}}^{p}}^{2}(x ; f) d x\left\{\lambda_{p, \boldsymbol{a}} \int_{0}^{1} V(x ; f) d x n^{-1}\right\}^{4 p}\right]^{1 /(4 p+1)} .
$$

\subsection{When Assumption A4 $[p]$ fails}

A sufficient condition for Assumption $\mathrm{A} 4[p]$ when $\mathcal{S}=[0, \infty)$ is that

$$
\text { M. for } j \in \mathbb{N}, \mu_{j}(K(\cdot ; x, \beta))=\sum_{k=\lceil j / 2\rceil}^{j} \zeta_{j, k} x^{j-k} \beta^{k}, \quad x \geq 0 \text {, }
$$

where $\zeta_{j, k}$ 's are some constants, independent of $\beta$ and $x$; see Examples 1 and 2 in Section 4 . However, that is not always true and careful considerations are required, on a case-by-case basis.

Though there is a slight difference, the moments may be rational functions:

$$
\operatorname{M} 1\left[p^{*}\right] . \quad \text { for } j \in \mathbb{N}, \mu_{j}(K(\cdot ; x, \beta))=\sum_{k=\lceil j / 2\rceil}^{j} \zeta_{j, k} x^{j-k} \beta^{k}+\chi_{\left\{j \geq p^{*}\right\}} \beta^{j} Q_{j}\left(\frac{x}{\beta}+1\right), \quad x \geq 0,
$$

where $Q_{j}(\cdot)$ is the rational function (independent of $\beta$ and $x$ ) with $\rho\left|Q_{j}(\rho)\right| \leq \bar{Q}_{j}<\infty$, having the form of

$$
Q_{j}(\rho)=\sum_{\ell_{1}=1}^{m_{1, j}} \sum_{\ell_{2}=1}^{m_{2, j}} \frac{\zeta_{j, \ell_{1}, \ell_{2}}}{\left(\rho+d_{\ell_{2}}\right)^{\ell_{1}}} \text { (type I) or } Q_{j}(\rho)=\frac{\left(\ell^{*}-1\right) \text { th polynomial in } \rho}{\ell^{*} \text { th polynomial in } \rho} \text { (type II) }
$$


for some natural numbers $p^{*}, m_{1, j}, m_{2, j}, \ell^{*}$ and real numbers $\zeta_{j, k}$ 's, $\zeta_{j, \ell_{1}, \ell_{2}}$ 's, $d_{\ell_{2}}$ 's.

More generally, the moments are not be expressed in terms of elementary functions, i.e.,

$$
\text { for } j \in \mathbb{N}, \mu_{j}(K(\cdot ; x, \beta))=(-x)^{j}+\sum_{\ell=1}^{j}{ }_{j} C_{\ell}(-x)^{j-\ell}(x+c \beta)^{\ell} g_{\ell}\left(\frac{x}{\beta}+c\right), \quad x \geq 0,
$$

for some constant $c \geq 1$ and a set of continuous functions $\left\{g_{\ell}\right\}$ on $(0, \infty)$, with $g_{\ell}(\rho) \rightarrow 1$ as $\rho \rightarrow \infty$. Here, we focus on the particular cases that are appeared in Section 4 (Examples 3-5):

$$
\text { (M2.1). } \quad g_{\ell}(\rho)=\left(1+\frac{1}{\rho}\right)^{\ell(\nu+\ell / 2)}, \quad(\mathrm{M} 2.2) . \quad g_{\ell}(\rho)=\frac{K_{\nu+\ell}(\rho)}{K_{\nu}(\rho)},
$$

where $\nu \notin\{1 / 2+M \mid M \in \mathbb{Z}\}$ ( $K_{\nu}$ stands for the modified Bessel function of the third kind), or

$$
(\mathrm{M} 2.3) . \quad g_{\ell}(\rho)= \begin{cases}\Gamma^{\ell-1}(\rho / \gamma) \frac{\Gamma((\rho+\ell) / \gamma)}{\Gamma^{\ell}((\rho+1) / \gamma)}, & \gamma \in(0, \infty) \backslash\{1 / M \mid M \in \mathbb{N}\}, \\ \Gamma^{\ell-1}((\rho+1) /|\gamma|) \frac{\left.\Gamma^{(}(\rho+1-\ell) /|\gamma|\right)}{\Gamma^{\ell}(\rho /|\gamma|)}, & \gamma \in(-\infty, 0) \backslash\{-1 / M \mid M \in \mathbb{N}\} .\end{cases}
$$

It is worth noting that $g_{1}(\rho) \not \equiv 1$ for either of (M2.1) or (M2.2), while $g_{1}(\rho) \equiv 1$ for (M2.3). Thus, we are now interested in the following structure:

$$
\text { for } j \in \mathbb{N}, \mu_{j}(K(\cdot ; x, \beta))=c^{j} \beta^{j}+\sum_{\ell=p^{*}}^{j}{ }_{j} C_{\ell}(-x)^{j-\ell}(x+c \beta)^{\ell}\left\{g_{\ell}\left(\frac{x}{\beta}+c\right)-1\right\}, \quad x \geq 0
$$

(we set $p^{*}=1$ for either of $(\mathrm{M} 2.1)$ or $(\mathrm{M} 2.2)$ and set $p^{*}=2$ for $(\mathrm{M} 2.3)$ ). Note that, for $j \in \mathbb{N}$,

$$
\mu_{j}(K(\cdot ; x, \beta))=\beta^{j}\left[c^{j}+\sum_{\ell=p^{*}}^{j}{ }_{j} C_{\ell}(-\kappa)^{j-\ell}(\kappa+c)^{\ell}\left\{g_{\ell}(\kappa+c)-1\right\}+\chi_{\{x=0\}} o(1)\right] \quad \text { for } x \in \mathcal{S}_{B, \beta, \kappa} .
$$

More importantly, using the large argument asymptotic expansion, we can verify that, for given $j \in \mathbb{N}$

$$
\mu_{j}(K(\cdot ; x, \beta))=\sum_{k=\lceil j / 2\rceil}^{j} \zeta_{j, k} x^{j-k} \beta^{k}+\chi_{\left\{j \geq p^{*}\right\}} \beta^{j} R_{j}\left(\frac{x}{\beta}+c\right), \quad \frac{x}{\beta}+c \geq M_{j},
$$

for some constants $M_{j}(>c)$ and $\zeta_{j, k}$ 's, independent of $\beta$ and $x$, where a set of continuous functions on $(0, \infty) ;\left\{R_{j}\right\}$ (independent of $\beta$ and $\left.x\right)$ satisfies $\rho\left|R_{j}(\rho)\right| \leq \bar{R}_{j}<\infty$. Here, (10) and (11) are gathered as $\mathrm{M} 2\left[p^{*}\right]$, which is an alternative to $\mathrm{M} 1\left[p^{*}\right]$.

Now, we know (e.g., Igarashi and Kakizawa (2014b, 2018a) and Igarashi (2016b)) that the (bias-uncorrected) asymmetric KDEs even in either of M1 $\left[p^{*}\right]$ or M2 $\left[p^{*}\right]$ (at least, (M2.1)-(M2.3)) have the MISEs of order $O\left(n^{-4 / 5}\right)$. Is it possible that using the bias correction methods reduces the convergence rate from $n^{-4 / 5}$, even if Assumption $\mathrm{A} 4[p]$ is replaced by $\mathrm{M} 1\left[p^{*}\right]$ or $\mathrm{M} 2\left[p^{*}\right]$ ? The answer is yes, but, the achievable rate when $f^{\left(p^{*}\right)}(0) \neq 0$ is shown to be $n^{-\left(4 p^{*}+2\right) /\left(4 p^{*}+3\right)}$, as follows: 
Case M1 $\left[p^{*}\right]: \operatorname{M} 1\left[p^{*}\right]$ implies that Assumption A4[p] holds for any integer $p ; 1 \leq p<p^{*}$; in this case, the results of the previous section are available. When $p \geq \max \left(2, p^{*}\right)$, we have

$$
\begin{aligned}
\operatorname{Bias}\left[\widehat{f}_{\beta, A D D_{\boldsymbol{a}}^{p}}(x)\right] & =\beta^{p} B_{p, \boldsymbol{a}}(x ; f)+\sum_{j=p^{*}}^{p} \beta^{j} f^{(j)}(x) \widetilde{B}_{j, \boldsymbol{a}}(x, \beta)+\mathcal{E}_{\beta, A D D_{\boldsymbol{a}}^{p}}(x), \quad x \geq 0, \\
\operatorname{Bias}\left[\widehat{f}_{\beta, \#_{\boldsymbol{a}}^{p}}(x)\right] & =\beta^{p} B_{\# \boldsymbol{a}}^{p}(x ; f)+\sum_{j=p^{*}}^{p} \beta^{j} f^{(j)}(x) \widetilde{B}_{j, \boldsymbol{a}}(x, \beta)+\mathcal{E}_{\beta, \#_{\boldsymbol{a}}^{p}}^{*}(x) \quad \text { for } x \in \mathcal{I}_{\iota, \iota_{0}}\left[r_{\beta}\right],
\end{aligned}
$$

where

$$
\begin{aligned}
\widetilde{B}_{j, \boldsymbol{a}}(x, \beta) & =\frac{1}{j !} \sum_{k=1}^{p} \frac{c_{k}(\boldsymbol{a})}{a_{k}^{j}} Q_{j}\left(\frac{a_{k} x}{\beta}+1\right), \quad p^{*} \leq j \leq p, \\
\mathcal{E}_{\beta, \# \boldsymbol{a}}^{*}(x) & = \begin{cases}\mathcal{E}_{\beta, T S_{a}^{p}}(x)+\chi_{\left\{p>p^{*}\right\}} O\left(\beta^{p^{*}+1-\iota_{0}}(1+x)\right), & \#=T S, \\
\mathcal{E}_{\beta, J F_{\boldsymbol{a}}^{p}}(x)+\chi_{\left\{p^{*}=1\right\}} O\left(\beta^{2-\iota_{0}}(1+x)\right), & \#=J F .\end{cases}
\end{aligned}
$$

Case $\mathrm{M} 2\left[p^{*}\right]\left(p^{*}=1\right.$ or $\left.p^{*}=2\right)$ : Let $p \in \mathbb{N} \backslash\{1\}$.

(i) When $p \geq \max \left(2, p^{*}\right)$, with $M \geq \max \left(M_{1}, \ldots, M_{2 p}, M_{2(p+1)}\right)$, we have

$$
\begin{array}{r}
\operatorname{Bias}\left[\widehat{f}_{\beta, A D D_{\boldsymbol{a}}^{p}}(x)\right]=\beta^{p} B_{p, \boldsymbol{a}}(x ; f)+\sum_{j=p^{*}}^{p} \beta^{j} f^{(j)}(x) \check{B}_{j, \boldsymbol{a}}(x, \beta)+\mathcal{E}_{\beta, A D D_{\boldsymbol{a}}^{p}}(x), \quad \frac{x}{\beta}+c \geq M, \\
\operatorname{Bias}\left[\widehat{f}_{\beta, \#_{\boldsymbol{a}}^{p}}(x)\right]=\beta^{p} B_{\#_{\boldsymbol{a}}^{p}}(x ; f)+\sum_{j=p^{*}}^{p} \beta^{j} f^{(j)}(x) \check{B}_{j, \boldsymbol{a}}(x, \beta)+\mathcal{E}_{\beta, \#_{\boldsymbol{a}}^{p}}^{*}(x) \\
\quad \text { for } x \in \mathcal{I}_{\iota, \iota_{0}}\left[r_{\beta}\right] \cap\left[(M-c) \beta, r_{\beta}\right],
\end{array}
$$

where

$$
\check{B}_{j, \boldsymbol{a}}(x, \beta)=\frac{1}{j !} \sum_{k=1}^{p} \frac{c_{k}(\boldsymbol{a})}{a_{k}^{j}} R_{j}\left(\frac{a_{k} x}{\beta}+c\right), \quad p^{*} \leq j \leq p .
$$

(ii) When $p=p^{*}=2$, we have

$$
\begin{aligned}
& \operatorname{Bias}\left[\widehat{f}_{\beta, A D D_{\left(a_{1}, a_{2}\right)}^{2}}(x)\right] \\
& =\beta^{2} \frac{f^{(2)}(0)}{2} \sum_{k=1}^{2} \frac{c_{k}\left(a_{1}, a_{2}\right)}{a_{k}^{2}}\left[c^{2}+\left(a_{k} \kappa+c\right)^{2}\left\{g_{2}\left(a_{k} \kappa+c\right)-1\right\}\right]+o\left(\beta^{2}\right) \quad \text { for } x \in \mathcal{S}_{B, \beta, \kappa}, \\
& \operatorname{Bias}\left[\widehat{f}_{\beta, \#_{\left(a_{1}, a_{2}\right)}^{2}}(x)\right] \\
& =\beta^{2}\left[\frac{f^{(2)}(0)}{2} \sum_{k=1}^{2} \frac{c_{k}\left(a_{1}, a_{2}\right)}{a_{k}^{2}}\left[c^{2}+\left(a_{k} \kappa+c\right)^{2}\left\{g_{2}\left(a_{k} \kappa+c\right)-1\right\}\right]+\frac{\left\{c f^{(1)}(0)\right\}^{2}}{2 f(0)\left(\prod_{k=1}^{2} a_{k}\right)^{\chi_{\{\#=T S\}}}}\right] \\
& \quad+o\left(\beta^{2}\right)+O\left(\beta^{-\iota_{0}} \sum_{k=1}^{2} V\left[\widehat{f}_{\beta / a_{k}}(x)\right]\right) \quad \text { for } x \in \mathcal{I}_{\iota, \iota_{0}}\left[r_{\beta}\right] \cap \mathcal{S}_{B, \beta, \kappa} .
\end{aligned}
$$


On the other hand, when $p>p^{*}$, we have

$$
\begin{aligned}
\operatorname{Bias}\left[\widehat{f}_{\beta, A D D_{a}^{p}}(x)\right]= & \beta^{p^{*}} \frac{f^{\left(p^{*}\right)}(0)}{p^{*} !} \sum_{k=1}^{p} \frac{c_{k}(\boldsymbol{a})}{a_{k}^{p^{*}}}\left(a_{k} \kappa+c\right)^{p^{*}}\left\{g_{p^{*}}\left(a_{k} \kappa+c\right)-1\right\}+o\left(\beta^{p^{*}}\right) \quad \text { for } x \in \mathcal{S}_{B, \beta, \kappa}, \\
\operatorname{Bias}\left[\widehat{f}_{\beta, \#_{a}^{p}}(x)\right]= & \beta^{p^{*}} \frac{f^{\left(p^{*}\right)}(0)}{p^{*} !} \sum_{k=1}^{p} \frac{c_{k}(\boldsymbol{a})}{a_{k}^{p^{*}}}\left(a_{k} \kappa+c\right)^{p^{*}}\left\{g_{p^{*}}\left(a_{k} \kappa+c\right)-1\right\} \\
& +o\left(\beta^{p^{*}}\right)+O\left(\beta^{-\iota_{0}} \sum_{k=1}^{p} V\left[\widehat{f}_{\beta / a_{k}}(x)\right]\right) \quad \text { for } x \in \mathcal{I}_{\iota, \iota_{0}}\left[r_{\beta}\right] \cap \mathcal{S}_{B, \beta, \kappa} .
\end{aligned}
$$

Thus, unless $f^{\left(p^{*}\right)}(0)=0$, the biases of the additive, TS-type, and JF-type estimators when $x \in \mathcal{S}_{B, \beta, \kappa}(\kappa>0)$ are of order $O\left(\beta^{\min \left(p^{*}, p\right)}\right)+\chi_{\{\#=T S, J F\}} O\left(\beta^{-\iota_{0}} \sum_{k=1}^{p} V\left[\widehat{f}_{\beta / a_{k}}(x)\right]\right)$. Also, we can see that the resulting integrated squared biases, when $p=p^{*}(\geq 2)$, are of order $O\left(\beta^{2 p}\right)+\chi_{\{\#=T S, J F\}} O\left(n^{-1} \beta^{-1 / 2}\right)$, and, when $p>p^{*}$, unless $f^{\left(p^{*}\right)}(0)=0$, they are of order $O\left(\beta^{\min \left(2 p^{*}+1,2 p\right)}\right)+\chi_{\{\#=T S, J F\}} O\left(n^{-1} \beta^{-1 / 2}\right)=O\left(\beta^{2 p^{*}+1}\right)+\chi_{\{\#=T S, J F\}} o\left(n^{-1} \beta^{-1 / 2}\right)$. Since the integrated variances are of order $O\left(n^{-1} \beta^{-1 / 2}\right)$, the MISEs, when $p=p^{*}(\geq 2)$, achieves the order $O\left(n^{-4 p /(4 p+1)}\right)$ by choosing $\beta \propto n^{-2 /(4 p+1)}$, and, when $p>p^{*}$, unless $f^{\left(p^{*}\right)}(0)=0$, they are of order $O\left(n^{-\left(4 p^{*}+2\right) /\left(4 p^{*}+3\right)}\right)$ at most ${ }^{[5]}$. Therefore, attention should be paid to this phenomenon under $\mathrm{M} 1\left[p^{*}\right]$ or $\mathrm{M} 2\left[p^{*}\right]$.

\section{Examples}

Associated with the special functions $B\left(\theta_{1}, \theta_{2}\right)=\int_{0}^{1} s^{\theta_{1}-1}(1-s)^{\theta_{2}-1} d s, \Gamma\left(\theta_{1}\right)=\int_{0}^{\infty} s^{\theta_{1}-1} e^{-s} d s$ (we have $B\left(\theta_{1}, \theta_{2}\right)=\Gamma\left(\theta_{1}\right) \Gamma\left(\theta_{2}\right) / \Gamma\left(\theta_{1}+\theta_{2}\right)$ ), and

$$
K_{\nu}\left(\theta_{1}\right)=\int_{0}^{\infty} \frac{s^{\nu-1}}{2} \exp \left\{-\frac{\theta_{1}}{2}\left(s+\frac{1}{s}\right)\right\} d s \quad\left(\text { note that } K_{ \pm 1 / 2}\left(\theta_{1}\right)=\left\{\pi /\left(2 \theta_{1}\right)\right\}^{1 / 2} e^{-\theta_{1}}\right),
$$

${ }^{[5]}$ Suppose that $p>p^{*}$.

The additive estimator has the MISE of order $O\left(n^{-\left(4 p^{*}+2\right) /\left(4 p^{*}+3\right)}\right)$ by choosing $\beta \propto n^{-2 /\left(4 p^{*}+3\right)}$.

The TS/JF-type estimators have the MISEs of order $O\left(n^{-\left(4 p^{*}+2\right) /\left(4 p^{*}+3\right)}\right)$ by choosing $\beta \propto n^{-2 /\left(4 p^{*}+3\right)}$ (if it is allowed). As in Remark 4, given $p \in \mathbb{N} \backslash\{1\}$ and $\left(\iota, \iota_{0}\right) \in \widetilde{I}_{p, \eta_{2 p}}$, where $\eta_{2 p} \in(0,1]$ is given in Assumption A5[p](ii),

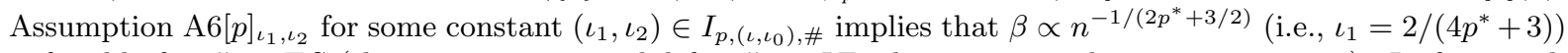
is feasible for \# $\#$ TS (the same remains valid for \# $=J F$ when $p=2$ and $0<a_{2}<a_{1}=1$ ). In fact, with $c_{p, \#}=0, \iota_{0} \in[0,1 / 2)$ (see (9)) implies $1 /\left(2 p^{*}+3 / 2\right)<1 / 2<1 /\left(1+2 \iota_{0}\right)$. On the other hand, for the JF-type (with $c_{p, J F}>0$ ), consider the pair $\left(p^{*}, p\right)$ with $p>p^{*}$, as follows: (i) if $p^{*}=1$, set $p=2$ (when $1=a_{1}<a_{2}$ ) or $p=3$, (ii) if $p^{*}$ is even, set $p=p^{*}+1$, and (iii) if $p^{*}(>2)$ is odd, set $p=p^{*}+1, p^{*}+2$. Then, as long as $\left(\iota, \iota_{0}\right) \in \widetilde{I}_{p, \eta_{2 p}}$ satisfies

we see that

$$
\iota_{0}<\frac{2 p^{*}+1 / 2-2 c_{p, J F}-c_{p, J F}(p-1) \iota}{c_{p, J F}(p-1)+2},
$$

$$
1+\left(\iota+\iota_{0}\right)(p-1)<\iota_{2}<\frac{2 p^{*}+1 / 2-c_{p, J F}-2 \iota_{0}}{c_{p, J F}}
$$

implies that $1 /\left(2 p^{*}+3 / 2\right)<1 /\left\{1+2 \iota_{0}+c_{p, J F}\left(1+\iota_{2}\right)\right\}$.

However, for other pair $\left(p^{*}, p\right)$ with $p>p^{*}$ (i.e., $\left(2 p^{*}+1 / 2-c_{p, J F}-2 \iota_{0}\right) / c_{p, J F}-1<0$ for $\left.\left(\iota, \iota_{0}\right) \in \widetilde{I}_{p, \eta_{2 p}}\right)$, the choice $\beta \propto n^{-1 /\left(2 p^{*}+3 / 2\right)}$ (i.e., $\left.\iota_{1}=2 /\left(4 p^{*}+3\right)\right)$ is not feasible, since, even if $1+\left(\iota+\iota_{0}\right)(p-1)<\iota_{2}$, it holds that, for $\left(\iota, \iota_{0}\right) \in \widetilde{I}_{p, \eta_{2 p}}$,

$$
\frac{2 p^{*}+1 / 2-c_{p, J F}-2 \iota_{0}}{c_{p, J F}}<1 \leq 1+\left(\iota+\iota_{0}\right)(p-1)<\iota_{2},
$$

hence, $1 /\left\{1+2 \iota_{0}+c_{p, J F}\left(1+\iota_{2}\right)\right\}<1 /\left(2 p^{*}+3 / 2\right)$; in this case, the MISEs of the TS/JF-type estimators are worse than $O\left(n^{-\left(4 p^{*}+2\right) /\left(4 p^{*}+3\right)}\right)$. 
where $\theta_{1}, \theta_{2}>0$ and $\nu \in \mathbb{R}$, the following densities are well-defined:

$$
\begin{aligned}
K_{\theta_{1}, \theta_{2}}^{(B)}(s) & =\frac{s^{\theta_{1}-1}(1-s)^{\theta_{2}-1}}{B\left(\theta_{1}, \theta_{2}\right)}, \quad 0 \leq s \leq 1, \\
K_{\theta_{1}, \theta_{2}}^{(G)}(s) & =\frac{\left(s / \theta_{2}\right)^{\theta_{1}-1} e^{-s / \theta_{2}}}{\theta_{2} \Gamma\left(\theta_{1}\right)}, \quad s \geq 0, \\
K_{\nu, \theta_{1}, \theta_{2}}^{(M B)}(s) & =\frac{\left(s / \theta_{2}\right)^{\nu-1}}{2 \theta_{2} K_{\nu}\left(\theta_{1}\right)} \exp \left\{-\frac{\theta_{1}}{2}\left(\frac{s}{\theta_{2}}+\frac{\theta_{2}}{s}\right)\right\}, \quad s>0 .
\end{aligned}
$$

Although the last density is known as generalized inverse Gaussian (IG) density (Jørgensen (1982)), it was renamed as "modified Bessel (MB) density" (Igarashi and Kakizawa (2014b)), noting that, in analogy to the gamma and beta densities $K_{\theta_{1}, \theta_{2}}^{(G)}(s)$ and $K_{\theta_{1}, \theta_{2}}^{(B)}(s)$, the MB function of the third kind, $K_{\nu}$, is the normalizing constant. Note that $K_{-1 / 2, \theta_{1}, \theta_{2}}^{(M B)}(s)$ and $K_{1 / 2, \theta_{1}, \theta_{2}}^{(M B)}(s)$ are IG and reciprocal IG (RIG) densities, respectively (Tweedie (1957)). A mixture of IG and RIG densities (MIG for short) is defined by

$$
K_{\theta_{1}, \theta_{2}}^{\left(M I G_{\varepsilon}\right)}(s)=\varepsilon K_{1 / 2, \theta_{1}, \theta_{2}}^{(M B)}(s)+(1-\varepsilon) K_{-1 / 2, \theta_{1}, \theta_{2}}^{(M B)}(s), \quad s>0, \theta_{1}, \theta_{2}>0,0 \leq \varepsilon \leq 1
$$

(Jørgensen et al. (1991)). Especially, $K_{\theta_{1}, \theta_{2}}^{\left(M I G_{1 / 2}\right)}(s)$ is known as Birnbaum-Saunders (BS) density (Birnbaum and Saunders (1969)).

Besides, we list two densities: One is a weighted log-normal $\left(\mathrm{LN}_{\nu}\right)$ density, defined by

$$
K_{\nu, \theta_{1}, \theta_{2}}^{(L N)}(s)=\frac{s^{\nu-1}}{\sqrt{2 \pi \theta_{2}}} \exp \left\{-\frac{\left(\log s-\theta_{1}\right)^{2}}{2 \theta_{2}}-\nu \theta_{1}-\frac{\nu^{2} \theta_{2}}{2}\right\}, \quad s>0, \nu \in \mathbb{R}, \theta_{1} \in \mathbb{R}, \theta_{2}>0 .
$$

Note that the $\mathrm{LN}_{0}$ density is the ordinary LN density and that $K_{\nu, \theta_{1}, \theta_{2}}^{(L N)}(s)=K_{0, \theta_{1}+\nu \theta_{2}, \theta_{2}}^{(L N)}(s)$. The other is Amoroso (Stacy or generalized gamma) density, defined by

$$
K_{\theta_{1}, \theta_{2}, \gamma}^{(A)}(s)=\frac{|\gamma|\left(s / \theta_{2}\right)^{\theta_{1} \gamma-1} e^{-\left(s / \theta_{2}\right)^{\gamma}}}{\theta_{2} \Gamma\left(\theta_{1}\right)}, \quad s \geq 0, \theta_{1}, \theta_{2}>0, \gamma \neq 0
$$

(Amoroso (1925), Stacy (1962), and Stacy and Mihram (1965)). The gamma density $K_{\theta_{1}, \theta_{2}}^{(G)}(s)$ is a core member with $\gamma=1$.

To build asymmetric KDEs from the above-mentioned densities, suitable parameterization is important, since, in principle, infinitely many parameterizations are possible ${ }^{[6]}$. In what follows, the parameter $\nu$ (or $\varepsilon, \gamma)$ is chosen in advance, independent of $\beta$ and $x$, unless otherwise stated. Then, we parameterize $\left(\theta_{1}, \theta_{2}\right)$ as a function of $\beta$ and $x$, in such a way that the resulting kernel concentrates around $s=x$ as $\beta \rightarrow 0$ (see the top of Section 2). By construction, the shape of such a kernel varies naturally according to the position $x \in \mathcal{S}$. This is a reason why the estimator is sometimes referred to as a varying asymmetric KDE.

\footnotetext{
${ }^{[6]}$ When $\mathcal{S}=[0, \infty)$, some existing estimators had the disadvantage that (i) $\widehat{f}_{\beta}(0)=0$ even if $f(0)>0$ (see Remark 7), and (ii) $\psi(x)=x^{J}$ for some $J \geq 2$ in Assumptions A1 and A2, hence, the asymptotic variance, being proportional to $f(x) / x^{J / 2}$, is not integrable on $[0, \infty)$, unless $f(x)=O\left(x^{\alpha}\right)$ for $\alpha>J / 2-1$ (in this case, $f(0)=0$ must be imposed).
} 
Example 1 The gamma KDE (Chen (2000))

$$
\widehat{f}_{\beta}^{(G)}(x)=\frac{1}{n} \sum_{i=1}^{n} K_{x / \beta+1, \beta}^{(G)}\left(X_{i}\right), \quad x \geq 0,
$$

satisfies Assumptions A1-A3 (see Igarashi and Kakizawa (2015)). We can prove, in supplemental issue (Supplemental appendix to "Higher-order bias corrections for kernel type density estimators on the unit or semi-infinite interval"), that, for any $j \in \mathbb{N}$,

$$
\mu_{j}\left(K_{x / \beta+1, \beta}^{(G)}(\cdot)\right)=\sum_{k=\lceil j / 2\rceil}^{j} \zeta_{j, k}^{(G)} x^{j-k} \beta^{k}, \quad x \geq 0,
$$

where $\zeta_{j, k}^{(G)}=\sum_{\ell=k}^{j}(-1)^{j+k-\ell}{ }_{j} C_{\ell} s(\ell+1, \ell+1-k)$ with the Stirling number of the first kind $s(\cdot, \cdot)$. Hence, Assumption A4 $[p]$ holds for any $p \in \mathbb{N}$. For example,

$$
\begin{aligned}
& \mu_{1}\left(K_{x / \beta+1, \beta}^{(G)}(\cdot)\right)=\beta, \quad \mu_{2}\left(K_{x / \beta+1, \beta}^{(G)}(\cdot)\right)=\beta x+2 \beta^{2}, \quad \mu_{3}\left(K_{x / \beta+1, \beta}^{(G)}(\cdot)\right)=5 \beta^{2} x+6 \beta^{3}, \\
& \mu_{4}\left(K_{x / \beta+1, \beta}^{(G)}(\cdot)\right)=3 \beta^{2} x^{2}+26 \beta^{3} x+24 \beta^{4}, \quad \mu_{5}\left(K_{x / \beta+1, \beta}^{(G)}(\cdot)\right)=35 \beta^{3} x^{2}+154 \beta^{4} x+120 \beta^{5}, \\
& \mu_{6}\left(K_{x / \beta+1, \beta}^{(G)}(\cdot)\right)=15 \beta^{3} x^{3}+340 \beta^{4} x^{2}+1044 \beta^{5} x+720 \beta^{6}, \quad \mu_{8}\left(K_{x / \beta+1, \beta}^{(G)}(\cdot)\right)=O\left(\beta^{4}(x+\beta)^{4}\right),
\end{aligned}
$$

can be derived using a computer algebra system (e.g., Maple).

Example 2 For every $\varepsilon \in[0,1]$, the $\mathrm{MIG}_{\varepsilon} \mathrm{KDE}$

$$
\widehat{f}_{\beta}^{\left(M I G_{\varepsilon}\right)}(x)=\frac{1}{n} \sum_{i=1}^{n} K_{x / \beta+1, x+\beta}^{\left(M I G_{\varepsilon}\right)}\left(X_{i}\right), \quad x \geq 0
$$

(the $\mathrm{MIG}_{\varepsilon}$ KDEs when $\varepsilon=0,1 / 2,1$ are referred to as the IG, BS, and RIG KDEs, respectively) satisfies Assumptions A1-A3 (see Igarashi and Kakizawa (2014b)). Given $j \in \mathbb{N}$,

$$
\mu_{j}\left(K_{x / \beta+1, x+\beta}^{\left(M I G_{\varepsilon}\right)}(\cdot)\right)=\sum_{k=\lceil j / 2\rceil}^{j} \zeta_{j, k}^{\left(M I G_{\varepsilon}\right)} x^{j-k} \beta^{k}, \quad x \geq 0,
$$

for some constants $\zeta_{j, k}^{\left(M I G_{\varepsilon}\right)}$,s, independent of $\beta$ and $x$ (we used a computer algebra system; Maple). For example,

$$
\begin{aligned}
& \mu_{1}\left(K_{x / \beta+1, x+\beta}^{\left(M I G_{\varepsilon}\right)}(\cdot)\right)=(\varepsilon+1) \beta, \quad \mu_{2}\left(K_{x / \beta+1, x+\beta}^{\left(M I G_{\varepsilon}\right)}(\cdot)\right)=\beta x+(5 \varepsilon+2) \beta^{2}, \\
& \mu_{3}\left(K_{x / \beta+1, x+\beta}^{\left(M I G_{\varepsilon}\right)}(\cdot)\right)=3(\varepsilon+2) \beta^{2} x+(30 \varepsilon+7) \beta^{3}, \\
& \mu_{4}\left(K_{x / \beta+1, x+\beta}^{\left(M I G_{\varepsilon}\right)}(\cdot)\right)=3 \beta^{2} x^{2}+3(14 \varepsilon+13) \beta^{3} x+(229 \varepsilon+37) \beta^{4}, \\
& \mu_{5}\left(K_{x / \beta+1, x+\beta}^{\left(M I G_{\varepsilon}\right)}(\cdot)\right)=15(\varepsilon+3) \beta^{3} x^{2}+5(105 \varepsilon+62) \beta^{4} x+(2165 \varepsilon+266) \beta^{5}, \\
& \mu_{6}\left(K_{x / \beta+1, x+\beta}^{\left(M I G_{\varepsilon}\right)}(\cdot)\right)=15 \beta^{3} x^{3}+45(9 \varepsilon+13) \beta^{4} x^{2}+30(233 \varepsilon+100) \beta^{5} x+(24576 \varepsilon+2431) \beta^{6}, \\
& \mu_{8}\left(K_{x / \beta+1, x+\beta}^{\left(M I G_{\varepsilon}\right)}(\cdot)\right)=O\left(\beta^{4}(x+\beta)^{4}\right) .
\end{aligned}
$$


Example 3 For every $\nu \in \mathbb{R}$, the $\mathrm{LN}_{\nu} \mathrm{KDE}$

$$
\widehat{f}_{\beta}^{\left(L N_{\nu}\right)}(x)=\frac{1}{n} \sum_{i=1}^{n} K_{\nu, \mu_{\beta}(x), \sigma_{\beta}^{2}(x)}^{(L N)}\left(X_{i}\right), \quad x \geq 0,
$$

satisfies Assumptions A1-A3, where $\mu_{\beta}(x)=\log (x+\beta), \sigma_{\beta}^{2}(x)=\log \{1+\beta /(x+\beta)\}$ (see Igarashi (2016b)). The $\mathrm{LN}_{\nu} \mathrm{KDE}, \nu=-1 / 2$ or $1 / 2$, satisfies M1[ $\left.p^{*}\right]$ of type I for $p^{*}=4$ or $p^{*}=2$, respectively (we used a computer algebra system; Maple). For example,

$$
\begin{aligned}
& \mu_{1}\left(K_{-1 / 2, \mu_{\beta}(x), \sigma_{\beta}^{2}(x)}^{(L N)}(\cdot)\right)=\beta, \quad \mu_{2}\left(K_{-1 / 2, \mu_{\beta}(x), \sigma_{\beta}^{2}(x)}^{(L N)}(\cdot)\right)=\beta x+2 \beta^{2}, \\
& \mu_{3}\left(K_{-1 / 2, \mu_{\beta}(x), \sigma_{\beta}^{2}(x)}^{(L N)}(\cdot)\right)=6 \beta^{2} x+8 \beta^{3}, \\
& \mu_{4}\left(K_{-1 / 2, \mu_{\beta}(x), \sigma_{\beta}^{2}(x)}^{(L N)}(\cdot)\right)=3 \beta^{2} x^{2}+40 \beta^{3} x+57 \beta^{4}+\frac{6 \beta^{5}}{x+\beta}+\frac{\beta^{6}}{(x+\beta)^{2}}, \\
& \mu_{1}\left(K_{1 / 2, \mu_{\beta}(x), \sigma_{\beta}^{2}(x)}^{(L N)}(\cdot)\right)=2 \beta, \quad \mu_{2}\left(K_{1 / 2, \mu_{\beta}(x), \sigma_{\beta}^{2}(x)}^{(L N)}(\cdot)\right)=\beta x+7 \beta^{2}+\frac{\beta^{3}}{x+\beta}
\end{aligned}
$$

(we see $m_{j}=j(j-3) / 2$ for $\nu=-1 / 2$ and $m_{j}=j(j-1) / 2$ for $\left.\nu=1 / 2\right)$. On the other hand, the $\mathrm{LN}_{J+1 / 2} \mathrm{KDE}$, where $J \in \mathbb{N}$, satisfies $\mathrm{M} 1\left[p^{*}\right]$ of type I for $p^{*}=1\left(\right.$ with $m_{1, j}=j(j+2 J-1) / 2$ and $m_{2, j}=1$ ), the $\mathrm{LN}_{-3 / 2} \mathrm{KDE}$ satisfies $\mathrm{M} 1\left[p^{*}\right]$ of type I for $p^{*}=1$ (with $m_{2, j}=2$ ), and the $\mathrm{LN}_{-(J+1 / 2)} \mathrm{KDE}$, where $J \in \mathbb{N} \backslash\{1\}$, satisfies M1 $\left[p^{*}\right]$ of type I for $p^{*}=1$ (with $m_{2, j}=1$ ). Also, the $\mathrm{LN}_{\nu} \mathrm{KDE}$, when $\nu$ is not an half-integer, satisfies (M2.1) with $c=1$.

Example 4 For every $\nu \in \mathbb{R}$, the $\mathrm{MB}_{\nu} \mathrm{KDE}$

$$
\widehat{f}_{\beta}^{\left(M B_{\nu}\right)}(x)=\frac{1}{n} \sum_{i=1}^{n} K_{\nu, x / \beta+1, x+\beta}^{(M B)}\left(X_{i}\right), \quad x \geq 0,
$$

satisfies Assumptions A1-A3 (see Igarashi and Kakizawa (2014b)). The case $\nu=-1 / 2$ or $1 / 2$ corresponds to the $\mathrm{MIG}_{\varepsilon} \mathrm{KDE}$, with $\varepsilon=0$ or 1 , respectively. The $\mathrm{MB}_{ \pm(J+1 / 2)}$ KDEs, where $J \in \mathbb{N}$, satisfy $\mathrm{M} 1\left[p^{*}\right]$ of type II for $p^{*}=1$ (with $\ell^{*}=J$ ). Also, the $\mathrm{MB}_{\nu} \mathrm{KDE}$, when $\nu$ is not an half-integer, satisfies (M2.2) with $c=1$.

Example 5 For every $\gamma \neq 0$, the Amoroso $\left(\mathrm{A}_{\gamma}\right) \mathrm{KDE}$

$$
\widehat{f}_{\beta}^{\left(A_{\gamma}\right)}(x)=\frac{1}{n} \sum_{i=1}^{n} K_{\alpha_{\gamma}(x / \beta+c), \beta \theta_{\gamma}(x / \beta+c), \gamma}^{(A)}\left(X_{i}\right), \quad x \geq 0
$$

(we choose $c=1$ when $\gamma>0$ or $c>1$ when $\gamma<0^{[7]}$ ) satisfies Assumptions A1-A3 (see Igarashi and Kakizawa (2018a)), where $\alpha_{\gamma}$ and $\theta_{\gamma}$ are continuous functions on $(0, \infty)$, defined by

$$
\alpha_{\gamma}(\rho)=\left\{\begin{array}{l}
\frac{\rho}{\gamma}, \quad \gamma>0, \\
\frac{\rho+1}{|\gamma|}, \quad \gamma<0,
\end{array} \quad \theta_{\gamma}(\rho)=\frac{\rho \Gamma\left(\alpha_{\gamma}(\rho)\right)}{\Gamma\left(\alpha_{\gamma}(\rho)+1 / \gamma\right)} .\right.
$$

\footnotetext{
${ }^{[7]}$ To ensure the existence of the higher-order moments of the resulting kernel, $c=1$ is not allowed for $\gamma<0$. More precisely, the $j$ th moment is well-defined if $j<x / \beta+c+1$ (see Igarashi and Kakizawa (2018a) for the details).
} 
The case $\gamma=1$ corresponds to the gamma KDE. The $\mathrm{A}_{1 / J} \mathrm{KDE}$, where $J \in \mathbb{N} \backslash\{1\}$, satisfies $\operatorname{M} 1\left[p^{*}\right]$ of type I for $p^{*}=2\left(\right.$ with $m_{1, j}=j-1$ and $m_{2, j}=J-1$ ), the $\mathrm{A}_{-1 / J} \mathrm{KDE}$, where $J \in \mathbb{N}$, satisfies M1 $\left[p^{*}\right]$ of type I for $p^{*}=2\left(\right.$ with $m_{1, j}=1$ and $m_{2, j}=J(j-1)$ ), and the $\mathrm{A}_{\gamma} \mathrm{KDE}$, except for $\gamma \in\{1 / M \mid M \in \mathbb{Z}, M \neq 0\}$, satisfies (M2.3).

Example 6 The beta KDE (Chen (1999))

$$
\widehat{f}_{\beta}^{(B)}(x)=\frac{1}{n} \sum_{i=1}^{n} K_{x / \beta+1,(1-x) / \beta+1}^{(B)}\left(X_{i}\right), \quad 0 \leq x \leq 1,
$$

satisfies Assumptions A1 and A2 (see Igarashi (2016a)). Given $j \in \mathbb{N}$,

$$
\mu_{j}\left(K_{x / \beta+1,(1-x) / \beta+1}^{(B)}(\cdot)\right)=\frac{\sum_{k=\lceil j / 2\rceil}^{j} \widetilde{\zeta}_{j, k}^{(B)}(x) \beta^{k}}{\prod_{k=1}^{j}\{1+(k+1) \beta\}}, \quad 0 \leq x \leq 1,
$$

for some polynomials $\widetilde{\zeta}_{j, k}^{(B)}(x)$ 's, independent of $\beta$ (we used a computer algebra system; Maple). Expanding the denominator, we have, for example, uniformly in $x \in[0,1]$,

$$
\begin{aligned}
& \mu_{1}\left(K_{x / \beta+1,(1-x) / \beta+1}^{(B)}(\cdot)\right)=\beta(1-2 x)+2 \beta^{2}\{-(1-2 x)\}+4 \beta^{3}(1-2 x)+O\left(\beta^{4}\right), \\
& \mu_{2}\left(K_{x / \beta+1,(1-x) / \beta+1}^{(B)}(\cdot)\right)=\beta x(1-x)+\beta^{2}\{2-11 x(1-x)\}+\beta^{3}\{-10+49 x(1-x)\}+O\left(\beta^{4}\right), \\
& \mu_{3}\left(K_{x / \beta+1,(1-x) / \beta+1}^{(B)}(\cdot)\right)=5 \beta^{2}(1-2 x) x(1-x)+3 \beta^{3}(1-2 x)\{2-19 x(1-x)\}+O\left(\beta^{4}\right), \\
& \mu_{4}\left(K_{x / \beta+1,(1-x) / \beta+1}^{(B)}(\cdot)\right)=3 \beta^{2} x^{2}(1-x)^{2}+2 \beta^{3} x(1-x)\{13-64 x(1-x)\}+O\left(\beta^{4}\right), \\
& \mu_{5}\left(K_{x / \beta+1,(1-x) / \beta+1}^{(B)}(\cdot)\right)=35 \beta^{3}(1-2 x) x^{2}(1-x)^{2}+O\left(\beta^{4}\right), \\
& \mu_{6}\left(K_{x / \beta+1,(1-x) / \beta+1}^{(B)}(\cdot)\right)=15 \beta^{3} x^{3}(1-x)^{3}+O\left(\beta^{4}\right), \quad \mu_{8}\left(K_{x / \beta+1,(1-x) / \beta+1}^{(B)}(\cdot)\right)=O\left(\beta^{4}\right) .
\end{aligned}
$$

Remark 6 (Two-regime type estimators) As variants of the gamma KDE, Chen (2000) and Igarashi and Kakizawa (2014b) introduced two-regime $\rho$-function

$$
\rho_{c}(t)=\left\{\begin{aligned}
t+c, & t \geq 2, \\
(c+1)\left(\frac{t}{2}\right)^{2 /(c+1)}+1, & t \in[0,2)
\end{aligned} \quad\right. \text { (say), }
$$

and suggested a class of modified gamma KDEs $\widehat{f}_{\beta}^{(G 2)}(x)=n^{-1} \sum_{i=1}^{n} K_{\rho_{c}(x / \beta), \beta}^{(G)}\left(X_{i}\right), x \geq 0$ $\left(c=1 / 4\right.$ is the best choice in the sense of the $O\left(n^{-4 / 5}\right)$-MISE). See also Igarashi and Kakizawa (2014b, 2018a) and Igarashi (2016b) for the related two-regime type MIG, $\mathrm{LN}_{\nu}$, and $\mathrm{A}_{\gamma} \mathrm{KDEs}$. However, it was revealed (see Igarashi and Kakizawa $(2015,2018 \mathrm{a}, \mathrm{c})$ ) that the two-regime type $\operatorname{KDEs}(\mathcal{S}=[0, \infty))$, after the bias corrections, have the MISEs of order $O\left(n^{-6 / 7}\right)$ at most, unless $f$ satisfies a shoulder condition $f^{(1)}(0)=0$. Similarly, the two-regime type modified beta KDE $\widehat{f}_{\beta}^{(B 2)}(x)=n^{-1} \sum_{i=1}^{n} K_{\rho_{c}(x / \beta), \rho_{c}((1-x) / \beta)}^{(B)}\left(X_{i}\right), 0 \leq x \leq 1$, after the bias corrections, has the MISE of order $O\left(n^{-6 / 7}\right)$ at most, unless $f^{(1)}(0)=f^{(1)}(1)=0$. 
Remark 7 (Bad estimators with $\widehat{f}_{\beta}(0)=0$ ) The variants of the IG, BS, and LN KDEs due to Jin and Kawczak (2003) and Scaillet (2004) were based on other parameterization of the IG, BS, and $\mathrm{LN}_{0}$ densities, i.e., $K_{1 /(\beta x), x}^{\left(M I G_{0}\right)}, K_{1 / \beta, x}^{\left(M I G_{1 / 2}\right)}$, and $K_{0, \log x, 4 \log (1+\beta)}^{(L N)}$, respectively. It may be true that these estimators, with/without bias corrections, work when $x>0$ (the details are omitted here). But, their kernels converge to zero as $x \rightarrow 0$; consequently, their estimators yield $\widehat{f}_{\beta}(0)=0$ regardless of $f(0)=0$ or $f(0)>0$. Also, the variant of the RIG KDE due to Scaillet (2004) (his kernel is $K_{x / \beta-1, x-\beta}^{\left(M I G_{1}\right)}$ ) had the downward bias $\left\{e^{-2(1-x / \beta)}-1\right\} f(0)+O(\beta)$ for $x<\beta$ (see Igarashi and Kakizawa (2014b)). These problems were obviously caused by the bad parameterization; when $x=0(x<\beta)$, their parameters lie outside the parameter spaces of the IG, BS, and $\mathrm{LN}_{0}$ (RIG) densities. Hence, these estimators are not appropriate for estimating a density with $f(0)>0$.

\section{Simulation studies}

We illustrate the finite sample performance of the bias-corrected estimators (4)-(6) for $p=2,3$ $(\boldsymbol{a}=(1, a)$ and $\boldsymbol{a}=(1, a, 1 / a)$ with $a=0.1,0.5,0.9)$, through the simulations, using the Amoroso $\left(\mathrm{A}_{\gamma}, \gamma= \pm 0.5, \pm 1, \pm 1.5, \pm 2\right), \mathrm{IG}, \mathrm{BS}, \mathrm{RIG}$, and $\mathrm{LN}_{-1 / 2}$ kernels (Examples 1, 2, 3, and 5). Note that, when $p=3$, the use of the gamma $\left(\mathrm{A}_{1}\right), \mathrm{IG}, \mathrm{BS}, \mathrm{RIG}$, and $\mathrm{LN}_{-1 / 2}$ kernels enables us to attain the convergence rate $n^{-12 / 13}$ of the MISE; however, in general, the use of the $\mathrm{A}_{\gamma}$ kernel, where $\gamma \in \mathbb{R} \backslash\{0,1\}$, yields the convergence rate $n^{-10 / 11}$ of the MISE, though it is faster than $n^{-8 / 9}$ for the previous paper (e.g., Igarashi and Kakizawa (2018a)).

We generated 1000 samples of $n=100,200$ from four densities:
A. $f(x)=\frac{1}{2}\left(\frac{e^{-x / 3}}{3}+\frac{x e^{-x / 3}}{9}\right)$,
B. $f(x)=\frac{e^{-x / 3}}{3}$,
C. $f(x)=\frac{1}{2}\left(\frac{e^{-x / 10}}{10}+x e^{-x}\right)$,
D. $f(x)=\frac{1}{2}\left[\frac{1}{\sqrt{2 \pi} 0.8 x} \exp \left\{-\frac{(\log x-1)^{2}}{2(0.8)^{2}}\right\}+\frac{1}{\sqrt{2 \pi} 0.4 x} \exp \left\{-\frac{(\log x-2)^{2}}{2(0.4)^{2}}\right\}\right]$,

and then calculated the integrated squared error (ISE) $I S E^{[k]}=\int_{\mathcal{S}}\left\{\hat{f}_{\beta}^{[k]}(x)-f(x)\right\}^{2} d x$ for the $k$ th sample. Each smoothing parameter $\beta$ was chosen using the least squared cross-validation method. We chose $\epsilon=0.000001 \times \beta^{2}$.

From Tables $1-4$, we observe that the average ISEs, $\sum_{k=1}^{1000} I S E^{[k]} / 1000$, decreased, as the sample size $n$ increased. For the cases $\mathrm{A}$ and $\mathrm{B}$, the average ISE of $\widehat{f}_{\beta, \#_{(1, a, 1 / a)}^{3}}$ decreased, as $a$ was close to one. Among the bias corrections using the IG, BS, and RIG kernels, the IG kernel had the best performance and the BS kernel was the second best. The IG kernel was inferior to the best implemented $\mathrm{A}_{\gamma}$ kernel. The $\mathrm{LN}_{-1 / 2}$ kernel had the similar performance to the IG kernel. Hereafter, we pay attention to the $\mathrm{A}_{\gamma}$ kernel $(n=200$ and $a=0.9)$.

- For the case A, $\widehat{f}_{\beta, \#_{(1, a, 1 / a)}^{3}}^{\left(A_{\gamma}\right)}, \#=A D D, T S, J F$, outperformed $\widehat{f}_{\beta, \#_{(1, a)}^{2}}^{\left(A_{\gamma}\right)}$, except for some kernels (overall, $\gamma<0$ was not good). Here, $\widehat{f}_{\beta, T S_{(1, a, 1 / a)}^{3}}^{\left(A_{1}\right)}$ had the best performance. 
- For the case $\mathrm{B}, \widehat{f}_{\beta, \#_{(1, a, 1 / a)}^{3}}^{\left(A_{\gamma}\right)}$, \# =ADD, TS, outperformed $\widehat{f}_{\beta, \#_{(1, a)}^{2}}^{\left(A_{\gamma}\right)}$, except for some kernels (overall, $\gamma<0$ was not good), and $\widehat{f}_{\beta, J F_{(1, a, 1 / a)}^{3}}^{\left(A_{ \pm 0.5}\right)}$ outperformed $\widehat{f}_{\beta, J F_{(1, a)}^{2}}^{\left(A_{ \pm 0.5}\right)}$. Here, $\widehat{f}_{\beta, T S_{(1, a, 1 / a)}^{3}}^{\left(A_{2}\right)}$ had the best performance.

- For the case $\mathrm{C}, \widehat{f}_{\beta, \#_{(1, a, 1 / a)}^{3}}^{\left(A_{\gamma}\right)}, \#=A D D, T S$, outperformed $\widehat{f}_{\beta, \#_{(1, a)}^{2}}^{\left(A_{\gamma}\right)}$ for $\gamma \leq 0.5$, and $\widehat{f}_{\beta, J F_{(1, a, 1 / a)}^{3}}^{\left(A_{\gamma}\right)}$ worked well. Here, $\widehat{f}_{\beta, T S_{(1, a, 1 / a)}^{3}}^{\left(A_{0.5)}\right)}$ had the best performance, and, among the $\mathrm{A}_{\gamma}$ 's except $\gamma=0.5, \widehat{f}_{\beta, \#_{(1, a, 1 / a)}^{3}}^{A_{-0.5)}}, \#=A D D, T S, J F$, worked well.

- For the case D (in this case, $f(0)=0), \widehat{f}_{\beta, \#_{(1, a)}^{2}}^{\left(A_{-0.5}\right)}$ and $\widehat{f}_{\beta, \#_{(1, a, 1 / a)}^{3}}^{\left(A_{-0.5}\right)}$, \# =ADD,TS,JF, worked well. Here, $\widehat{f}_{\beta, A D D_{(1, a)}^{2}}^{\left(A_{-0.5}\right)}$ had the best performance and $\widehat{f}_{\beta, A D D_{(1, a, 1 / a)}^{3}}^{\left(A_{-0.5}\right)}$ was the second best. Note that a better performance of the exponent $\gamma<0$ is also found in other contexts (Kakizawa and Igarashi (2017) and Igarashi and Kakizawa (2018a,b,c)).

\section{Concluding remarks}

\section{1. $\quad$ Limiting estimator}

Given a positive vector $\boldsymbol{a}=\left(1, H_{2}(a), \ldots, H_{p}(a)\right)^{\prime}$, such that $\lim _{a \rightarrow 1} H_{k}(a)=1$ for $k=2, \ldots, p$, we guess that $\lim _{a \rightarrow 1} \widehat{f}_{\beta, \#_{a}^{p}}(x)$, \# $=A D D, T S, J F$, are also the bias-corrected asymmetric KDEs, provided that, for the TS-type and JF-type, $\epsilon>0$ is independent of $a \in(0,1)$. In fact, extending Igarashi and Kakizawa (2015, 2018b) and Igarashi (2016a) for $p=2$, we can construct the limiting estimators $\lim _{a \rightarrow 1} \widehat{f}_{\beta, \#_{(1, a, 1 / a)}^{3}}(x)=\widehat{f}_{\beta, \#_{(1,1,1)}^{3}}(x)$ (say), where

$$
\begin{aligned}
\widehat{f}_{\beta, A D D_{(1,1,1)}^{3}(x)} & =\widehat{f}_{\beta}(x)-\beta \frac{\partial}{\partial \beta} \widehat{f}_{\beta}(x)+\frac{1}{2} \beta^{2} \frac{\partial^{2}}{\partial \beta^{2}} \widehat{f}_{\beta}(x), \\
\widehat{f}_{\beta, T S_{(1,1,1)}^{3}}(x) & =\left\{\widehat{f}_{\beta}(x)+\epsilon\right\} \exp \left[\frac{\widehat{f}_{\beta, A D D_{(1,1,1)}^{3}}(x)}{\widehat{f}_{\beta}(x)+\epsilon}-1-\frac{\left\{\beta \frac{\partial}{\partial \beta} \widehat{f}_{\beta}(x)+\epsilon\right\}^{2}}{2\left\{\widehat{f}_{\beta}(x)+\epsilon\right\}^{2}}\right], \\
\widehat{f}_{\beta, J F_{(1,1,1)}^{3}}(x) & =\left\{\widehat{f}_{\beta}(x)+\epsilon\right\} \exp \left[\sum_{j=1}^{2} \frac{(-1)^{j-1}}{j}\left\{\frac{\widehat{f}_{\beta, A D D_{(1,1,1)}^{3}}(x)}{\widehat{f}_{\beta}(x)+\epsilon}-1\right\}^{j}\right] .
\end{aligned}
$$

\subsection{Case $\mathcal{S}=\mathbb{R}$}

Suppose that $\sup p(f)=\mathbb{R}$. If $f$ is $2 p$ times continuously differentiable for some $p \in \mathbb{N} \backslash\{1\}$, the classical Rosenblatt-Parzen KDE $\hat{f}_{h}^{\left(K_{[2]}\right)}(x)=(n h)^{-1} \sum_{i=1}^{n} K_{[2]}\left(\left(x-X_{i}\right) / h\right)$, using a symmetric second-order kernel $K_{[2]}$, yields

$$
E\left[\widehat{f}_{h}^{\left(K_{[2]}\right)}(x)\right]=f(x)+\sum_{k=1}^{p} h^{2 k} \frac{f^{(2 k)}(x)}{(2 k) !} \int_{-\infty}^{\infty} z^{2 k} K_{[2]}(z) d z+o\left(h^{2 p}\right)
$$

(in this case, $\left.\beta=h^{2}\right)$. For a given positive vector $\boldsymbol{a}=\left(a_{1}, \ldots, a_{p}\right)^{\prime}$, such that the $a_{k}$ 's are distinct, let $\boldsymbol{a}^{2}=\left(a_{1}^{2}, \ldots, a_{p}^{2}\right)^{\prime}$. The bias-corrected KDE, $(n h)^{-1} \sum_{i=1}^{n} K_{[2 p], \boldsymbol{a}}\left(\left(x-X_{i}\right) / h\right)$, can 
Table 1: Case A. The average ISEs $\times 10^{6}$ of estimators with/without bias corrections, where $A_{\gamma}, I G, B S, R I G$, and $L N_{-1 / 2}$ stand for the asymmetric KDEs (see Examples 1, 2, 3, and 5).

The number in the parentheses stands for the standard deviation $\times 10^{6}$ of the ISEs.

\begin{tabular}{|c|c|c|c|c|c|c|c|c|c|c|c|c|c|c|}
\hline & $p$ & $a$ & $A_{2}$ & $A_{1.5}$ & $A_{1}$ & $A_{0.5}$ & $A_{-0.5}$ & $A_{-1}$ & $A_{-1.5}$ & $A_{-2}$ & $I G$ & $B S$ & $R I G$ & $L N_{-1 / 2}$ \\
\hline \multicolumn{3}{|c|}{ original } & 3389 & 3263 & 3006 & 2914 & 3201 & 3392 & 3735 & 4135 & 3316 & 3539 & 3751 & 3288 \\
\hline & & & $(2946)$ & $(3216)$ & $(3144)$ & $(3206)$ & $(3235)$ & (2898) & $(2674)$ & $(2941)$ & $(3346)$ & $(3411)$ & $(3468)$ & $(3317)$ \\
\hline \multirow[t]{6}{*}{$A D D$} & 2 & 0.1 & 2715 & 2607 & 2498 & 2595 & 2782 & 2750 & 3099 & 3419 & 2627 & 2944 & 3071 & 2564 \\
\hline & & & $(3023)$ & (2994) & $(3129)$ & $(3061)$ & $(2931)$ & $(2606)$ & $(2595)$ & $(2666)$ & $(2843)$ & $(3396)$ & $(3312)$ & $(2756)$ \\
\hline & & 0.5 & 2369 & 2356 & 2255 & 2382 & 2531 & 2519 & 2905 & 3344 & 2352 & 2574 & 2738 & 2328 \\
\hline & & & $(3005)$ & $(3185)$ & $(3023)$ & $(2826)$ & $(2405)$ & $(2279)$ & $(2362)$ & $(2572)$ & $(2615)$ & $(2992)$ & $(3271)$ & $(2649)$ \\
\hline & & 0.9 & 2268 & 2326 & 2207 & 2372 & 2511 & 2565 & 2956 & 3476 & 2336 & 2564 & 2713 & 2327 \\
\hline & & & $(2627)$ & $(3185)$ & $(2856)$ & $(2781)$ & $(2262)$ & $(2432)$ & $(2442)$ & $(2700)$ & $(2587)$ & $(3042)$ & $(3265)$ & $(2682)$ \\
\hline \multirow[t]{6}{*}{$A D D$} & 3 & 0.1 & 3533 & 3182 & 2866 & 2773 & 3193 & 3675 & 4237 & 4863 & 3336 & 2975 & 2954 & 3332 \\
\hline & & & $(3758)$ & (3619) & $(3584)$ & $(3630)$ & (3633) & $(3771)$ & $(4052)$ & $(4380)$ & (3809) & $(3585)$ & $(3533)$ & $(3841)$ \\
\hline & & 0.5 & 2221 & 2162 & 2000 & 2298 & 2502 & 2626 & 3311 & 4118 & 2281 & 2413 & 2513 & 2241 \\
\hline & & & $(3074)$ & $(3374)$ & $(2786)$ & $(2878)$ & $(2710)$ & $(2794)$ & $(3047)$ & $(3622)$ & $(3047)$ & $(3052)$ & $(3258)$ & (2994) \\
\hline & & 0.9 & 2090 & 2034 & 2004 & 2314 & 2446 & 2568 & 3455 & 4465 & 2176 & 2299 & 2392 & 2138 \\
\hline & & & $(2814)$ & (2971) & $(2784)$ & $(2890)$ & $(2411)$ & $(2450)$ & $(2996)$ & $(3494)$ & (2661) & $(2858)$ & $(3142)$ & $(2752)$ \\
\hline \multirow[t]{6}{*}{$T S$} & 2 & 0.1 & 2434 & 2396 & 2334 & 2600 & 2653 & 2329 & 2500 & 2752 & 2314 & 2600 & 2717 & 2295 \\
\hline & & & $(3047)$ & $(3023)$ & $(3016)$ & $(3101)$ & $(2465)$ & $(2270)$ & $(2542)$ & $(2945)$ & $(2616)$ & (2988) & $(3162)$ & (2695) \\
\hline & & 0.5 & 2061 & 2164 & 2071 & 2407 & 2557 & 2298 & 2767 & 3376 & 2202 & 2386 & 2477 & 2121 \\
\hline & & & $(2459)$ & (3016) & $(2690)$ & $(2808)$ & $(2242)$ & $(2327)$ & $(2630)$ & $(2963)$ & $(2478)$ & $(2912)$ & $(3096)$ & $(2549)$ \\
\hline & & 0.9 & 2026 & 2091 & 2066 & 2411 & 2606 & 2390 & 2967 & 3665 & 2165 & 2369 & 2470 & 2117 \\
\hline & & & $(2383)$ & $(2789)$ & $(2702)$ & $(2882)$ & $(2303)$ & $(2462)$ & $(2782)$ & $(3082)$ & $(2581)$ & $(2934)$ & $(3163)$ & $(2602)$ \\
\hline \multirow[t]{6}{*}{$T S$} & 3 & 0.1 & 3864 & 3426 & 2997 & 2832 & 3307 & 3905 & 4687 & 5457 & 3545 & 3143 & 2990 & 3551 \\
\hline & & & (3911) & $(3769)$ & $(3637)$ & $(3658)$ & $(3696)$ & $(3826)$ & $(4282)$ & $(4742)$ & (3929) & $(3825)$ & $(3672)$ & $(3946)$ \\
\hline & & 0.5 & 2593 & 2303 & 1989 & 2321 & 2631 & 2898 & 3777 & 4862 & 2456 & 2310 & 2355 & 2459 \\
\hline & & & $(3315)$ & (3409) & $(2969)$ & $(2862)$ & $(2739)$ & $(2879)$ & $(3218)$ & $(3788)$ & $(3040)$ & $(3218)$ & $(3172)$ & $(3105)$ \\
\hline & & 0.9 & 2574 & 2146 & $\underline{1933}$ & 2342 & 2614 & 2820 & 3941 & 5242 & 2380 & 2259 & 2313 & 2375 \\
\hline & & & $(3246)$ & (2979) & (2919) & $(2862)$ & $(2690)$ & (2639) & $(3251)$ & $(3842)$ & (2979) & $(3106)$ & (2998) & $(3041)$ \\
\hline \multirow[t]{6}{*}{$J F$} & 2 & 0.1 & 2692 & 2600 & 2483 & 2591 & 2753 & 2738 & 3088 & 3364 & 2615 & 2949 & 3044 & 2556 \\
\hline & & & $(3010)$ & (3009) & $(3054)$ & $(3063)$ & $(2823)$ & $(2617)$ & $(2610)$ & $(2585)$ & $(2842)$ & $(3413)$ & $(3291)$ & $(2766)$ \\
\hline & & 0.5 & 2226 & 2272 & 2150 & 2388 & 2531 & 2464 & 2838 & 3279 & 2266 & 2501 & 2643 & 2243 \\
\hline & & & $(2795)$ & $(3141)$ & $(2849)$ & $(2819)$ & $(2272)$ & $(2317)$ & $(2436)$ & $(2629)$ & $(2492)$ & $(2986)$ & $(3182)$ & (2618) \\
\hline & & 0.9 & 2029 & 2140 & 2069 & 2401 & 2581 & 2384 & 2898 & 3571 & 2198 & 2413 & 2533 & 2164 \\
\hline & & & $(2377)$ & (3009) & $(2680)$ & $(2839)$ & $(2249)$ & $(2440)$ & (2624) & $(3034)$ & $(2574)$ & $(2989)$ & $(3202)$ & (2619) \\
\hline \multirow[t]{6}{*}{$J F$} & 3 & 0.1 & 3435 & 3147 & 2807 & 2634 & 2748 & 3215 & 3805 & 4262 & 3013 & 3400 & 3659 & 3045 \\
\hline & & & $(2975)$ & (2966) & $(3219)$ & $(3446)$ & $(3380)$ & (2995) & $(2942)$ & $(2912)$ & (3228) & $(3149)$ & $(3232)$ & $(3182)$ \\
\hline & & 0.5 & 2368 & 2328 & 2154 & 2343 & 2455 & 2663 & 3136 & 3617 & 2396 & 2537 & 2694 & 2401 \\
\hline & & & $(2960)$ & $(3325)$ & $(2877)$ & $(2856)$ & $(2520)$ & $(2590)$ & $(2600)$ & $(2751)$ & (2833) & $(3082)$ & $(3205)$ & $(2849)$ \\
\hline & & 0.9 & 2139 & 2174 & 2112 & 2332 & 2464 & 2592 & 3124 & 3667 & 2297 & 2445 & 2622 & 2325 \\
\hline & & & $(2615)$ & $(3166)$ & (2933) & $(2876)$ & $(2467)$ & $(2411)$ & $(2527)$ & $(2774)$ & $(2527)$ & $(3033)$ & $(3412)$ & $(2754)$ \\
\hline
\end{tabular}

The underlined or double-underlined number indicates the smallest or second smallest average ISE, respectively. 
Table 1: (continued).

\begin{tabular}{|c|c|c|c|c|c|c|c|c|c|c|c|c|c|c|}
\hline & $p$ & $a$ & $A_{2}$ & $A_{1.5}$ & $A_{1}$ & $A_{0.5}$ & $A_{-0.5}$ & $A_{-1}$ & $A_{-1.5}$ & $A_{-2}$ & $I G$ & $B S$ & $R I G$ & $L N_{-1}$ \\
\hline \multirow{2}{*}{\multicolumn{3}{|c|}{ original }} & 2107 & 2005 & 1802 & 1690 & 1831 & 2048 & 2283 & 2489 & 1971 & 2157 & 2329 & 1942 \\
\hline & & & (2285) & $(2353)$ & $(2171)$ & $(2077)$ & (2098) & $(2044)$ & $(2018)$ & (1913) & $(2229)$ & $(2372)$ & $(2475)$ & (2166) \\
\hline \multirow[t]{6}{*}{$A D D$} & 2 & 0.1 & 1579 & 1532 & 1458 & 1421 & 1607 & 1650 & 1779 & 1988 & 1566 & 1724 & 1763 & 1538 \\
\hline & & & (1884) & $(2155)$ & $(2185)$ & $(1739)$ & $(2011)$ & $(1932)$ & (1508) & (1902) & $(2153)$ & $(2337)$ & $(2235)$ & (2134) \\
\hline & & 0.5 & 1323 & 1282 & 1256 & 1333 & 1473 & 1465 & 1624 & 1850 & 1361 & 1516 & 1599 & 1363 \\
\hline & & & (1799) & (1793) & $(1805)$ & $(1724)$ & (1787) & $(1580)$ & (1259) & $(1320)$ & (1884) & $(2257)$ & $(2315)$ & (1991) \\
\hline & & 0.9 & 1301 & 1265 & 1232 & 1316 & 1468 & 1437 & & 1886 & 30 & 1505 & 89 & \\
\hline & & & (1717) & $(1747)$ & (1734) & (1728) & (1664) & (1300) & $(1252)$ & (1321) & (1848) & $(2273)$ & (2330) & (1950) \\
\hline \multirow[t]{6}{*}{$A D D$} & 3 & 0.1 & 1879 & 1723 & 1562 & 1460 & 1663 & 1924 & 2204 & 2538 & 1767 & 1662 & 1733 & 1768 \\
\hline & & & (2531) & $(2418)$ & $(2350)$ & $(2194)$ & $(2136)$ & $(2370)$ & $(2330)$ & $(2617)$ & $(2397)$ & $(2406)$ & $(2346)$ & 8) \\
\hline & & 0.5 & 1255 & 1176 & 1165 & 1267 & 1466 & 1421 & 1744 & 2083 & 1244 & 1355 & 1401 & 1239 \\
\hline & & & (1904) & (1798) & $(1796)$ & $(1622)$ & $(1750)$ & $(1442)$ & (1629) & $(1856)$ & (1689) & $(2061)$ & $(2010)$ & 9) \\
\hline & & 0.9 & 1192 & 1122 & 153 & 1232 & 1417 & 1401 & 1770 & 2282 & 1220 & 1296 & 1372 & 20 \\
\hline & & & (1429) & (1733) & (1819) & $(1455)$ & $(1420)$ & (1380) & $(1546)$ & (1869) & (1443) & (1812) & (1996) & (1745) \\
\hline \multirow[t]{6}{*}{$T$} & 2 & 0.1 & 381 & 1384 & 1352 & 1432 & 157 & 1404 & 136 & 1445 & 13 & 1560 & 1571 & \\
\hline & & & (1807) & (2093) & (2119) & (1798) & (1950) & (1813) & (1324) & $(1382)$ & (1995) & $(2280)$ & (2189) & (1923) \\
\hline & & 0.5 & 1239 & 1217 & 1198 & 1333 & 147 & 1296 & 141 & $16^{\prime}$ & 1245 & 1378 & 1435 & 1221 \\
\hline & & & (1730) & $(1760)$ & (1749) & $(1711)$ & $(1529)$ & (1238) & $(1286)$ & $(1470)$ & (1619) & $(2006)$ & $(2134)$ & (1629) \\
\hline & & 0. & 1202 & 1182 & 1180 & 1313 & 1475 & 1320 & 1512 & 1826 & 1238 & 1374 & 1421 & 8 \\
\hline & & & (1714) & $(1717)$ & $(1729)$ & $(1555)$ & $(1534)$ & (1318) & $(1401)$ & $(1533)$ & $(1579)$ & $(2025)$ & $(2131)$ & 3) \\
\hline \multirow[t]{6}{*}{$T S$} & 3 & 0.1 & 2029 & 1814 & 1616 & 1495 & 1692 & 2005 & 2388 & 2749 & 1844 & 1673 & 1614 & 1853 \\
\hline & & & (2580) & $(2422)$ & $(2366)$ & $(2216)$ & $(2106)$ & $(2326)$ & $(2513)$ & (2579) & $(2405)$ & $(2437)$ & $(2396)$ & (2434) \\
\hline & & 0.5 & 1365 & 1219 & $\underline{\underline{1106}}$ & 1304 & 1514 & 1490 & 1908 & 2403 & 1262 & 1299 & 1364 & 34 \\
\hline & & & (1682) & (1904) & (1791) & $(1612)$ & (1836) & (1473) & (1571) & (1884) & (1719) & (1858) & $(2121)$ & (1713) \\
\hline & & 0.9 & 1331 & 1173 & $\underline{1080}$ & 1257 & 1480 & 1478 & 1983 & 2659 & 1232 & 1272 & 1310 & 1229 \\
\hline & & & (1518) & (1823) & $(1750)$ & (1413) & (1423) & (1367) & (1616) & (1996) & (1634) & (1811) & (1895) & (1619) \\
\hline \multirow{6}{*}{\multicolumn{2}{|c|}{$J F$}} & 0.1 & 1570 & 1512 & 1456 & 1419 & 1600 & 1641 & 1767 & 1963 & 1559 & 1728 & 1775 & 1533 \\
\hline & & & (1907) & $(2131)$ & $(2180)$ & (1739) & (1991) & (1931) & $(1505)$ & $(1873)$ & $(2146)$ & $(2357)$ & $(2353)$ & (2128) \\
\hline & & 0.5 & 1280 & 1244 & 1226 & 1330 & 1480 & 1382 & 1551 & 1796 & 1303 & 1465 & 1537 & 1302 \\
\hline & & & (1779) & $(1757)$ & (1771) & $(1715)$ & $(1760)$ & (1285) & (1224) & (1398) & (1657) & (2198) & $(2243)$ & (1751) \\
\hline & & 0.9 & 1208 & 1185 & 1194 & 1311 & 1466 & 1343 & 1516 & 1802 & 1248 & 1389 & 1441 & 1247 \\
\hline & & & (1713) & $(1704)$ & $(1772)$ & $(1556)$ & $(1526)$ & $(1312)$ & (1363) & (1509) & $(1585)$ & $(2036)$ & $(2149)$ & (1687) \\
\hline \multirow[t]{6}{*}{$J F$} & 3 & 0.1 & 1926 & 1855 & 1656 & 1484 & 1587 & 1891 & 2135 & 2351 & 1808 & 1993 & 2085 & 1793 \\
\hline & & & (2021) & $(2420)$ & (2211) & (2139) & $(2106)$ & (2064) & (1881) & $(1792)$ & $(2332)$ & $(2464)$ & $(2383)$ & (2201) \\
\hline & & 0.5 & 1287 & 1225 & 1205 & 1291 & 1447 & 1489 & 1694 & 1951 & 1341 & 1462 & 1493 & 1338 \\
\hline & & & (1819) & $(1789)$ & (1809) & $(1773)$ & $(1693)$ & $(1590)$ & (1298) & $(1521)$ & (1921) & $(2292)$ & $(2044)$ & (1978) \\
\hline & & 0.9 & 1226 & 1157 & 1182 & 1238 & 1397 & 1466 & 1705 & 1997 & 1245 & 1400 & 1416 & 1266 \\
\hline & & & (1798) & $726)$ & (1827) & (1457) & (1387) & $(1552)$ & (1253) & (1499) & (1379) & (2168) & (1983) & (1716) \\
\hline
\end{tabular}


Table 2: Case B. The average ISEs $\times 10^{6}$ of estimators with/without bias corrections, where $A_{\gamma}, I G, B S, R I G$, and $L N_{-1 / 2}$ stand for the asymmetric KDEs (see Examples 1, 2, 3, and 5).

The number in the parentheses stands for the standard deviation $\times 10^{6}$ of the ISEs.

\begin{tabular}{|c|c|c|c|c|c|c|c|c|c|c|c|c|c|c|}
\hline & $p$ & $a$ & $A_{2}$ & $A_{1.5}$ & $A_{1}$ & $A_{0.5}$ & $A_{-0.5}$ & $A_{-1}$ & $A_{-1.5}$ & $A_{-2}$ & $I G$ & $B S$ & $R I G$ & $L N_{-1 / 2}$ \\
\hline \multicolumn{3}{|c|}{ original } & 6452 & 6480 & 6049 & 5578 & 5924 & 6652 & 7505 & 7983 & 6565 & 7184 & 7466 & 6478 \\
\hline & & & $(5871)$ & $(7440)$ & $(7483)$ & $(7131)$ & (6366) & $(6154)$ & $(6561)$ & $(6147)$ & $(7350)$ & $(8495)$ & $(7694)$ & (7071) \\
\hline \multirow[t]{6}{*}{$A D D$} & 20 & 0.1 & 5247 & 5315 & 4989 & 5022 & 5215 & 5615 & 6042 & 6552 & 5220 & 5827 & 5942 & 5239 \\
\hline & & & (5829) & (7027) & $(6508)$ & $(7021)$ & (6105) & $(5991)$ & $(5760)$ & $(5512)$ & $(6176)$ & $(7052)$ & $(6380)$ & (6481) \\
\hline & & 0.5 & 4142 & 4365 & 4490 & 4686 & 4684 & 4707 & 5340 & 5912 & 4537 & 5046 & 5400 & 4419 \\
\hline & & & (4704) & $(5369)$ & $(5935)$ & $(6591)$ & (5231) & $(4215)$ & $(4569)$ & $(4675)$ & (4995) & $(5758)$ & $(6548)$ & $(5051)$ \\
\hline & & 0.9 & 4069 & 4312 & 4368 & 4659 & 4693 & 4662 & 5337 & 5940 & 4505 & 5013 & 5208 & 4337 \\
\hline & & & (4699) & $(5469)$ & $(5789)$ & $(6653)$ & (5309) & $(4154)$ & $(4556)$ & $(4668)$ & $(5644)$ & $(5811)$ & $(6185)$ & $(4881)$ \\
\hline \multirow[t]{6}{*}{$A D D$} & 30 & 0.1 & 5417 & 5211 & 4748 & 4816 & 5292 & 5966 & 6757 & 7402 & 5431 & 5794 & 5874 & 5393 \\
\hline & & & (6988) & $(7563)$ & $(6885)$ & (7329) & (6916) & $(7284)$ & $(7439)$ & $(7606)$ & $(7545)$ & $(7618)$ & $(6431)$ & $(7726)$ \\
\hline & & 0.5 & 3697 & 3819 & 4109 & 4532 & 4613 & 4450 & 5132 & 5926 & 4129 & 4543 & 4495 & 4039 \\
\hline & & & (5053) & $(5180)$ & $(5736)$ & $(6727)$ & (5319) & $(4371)$ & $(5057)$ & $(5721)$ & (4401) & $(5325)$ & $(5297)$ & (4667) \\
\hline & & 0.9 & 3234 & 3627 & 3893 & 4369 & 4589 & 4258 & 5012 & 6105 & 4007 & 4447 & 4324 & 3805 \\
\hline & & & (3697) & (5093) & $(5439)$ & $(6268)$ & $(5429)$ & $(4240)$ & $(4902)$ & $(5671)$ & $(4310)$ & $(5362)$ & $(5313)$ & $(4336)$ \\
\hline \multirow[t]{6}{*}{$T S$} & 20 & 0.1 & 4577 & 4593 & 4681 & 4980 & 4983 & 4496 & 4619 & 4870 & 4627 & 5312 & 5344 & 4638 \\
\hline & & & $(5600)$ & $(6078)$ & $(6526)$ & (7108) & $(5656)$ & $(4617)$ & $(4379)$ & $(4458)$ & $(5460)$ & $(6822)$ & $(6464)$ & $(6346)$ \\
\hline & & 0.5 & 3583 & 3869 & 4081 & 4529 & 4674 & 4069 & 4447 & 5130 & 3926 & 4588 & 4490 & 3753 \\
\hline & & & (4768) & (5368) & $(5697)$ & $(6471)$ & (5223) & $(4352)$ & (3999) & $(4223)$ & $(4352)$ & $(5425)$ & $(5480)$ & (4394) \\
\hline & & 0.9 & 3498 & 3785 & 4013 & 4503 & 4714 & 4124 & 4765 & 5547 & 3906 & 4501 & 4432 & 3730 \\
\hline & & & (4686) & $(5342)$ & $(5594)$ & $(6483)$ & $(5304)$ & $(4172)$ & $(4793)$ & $(5000)$ & $(4330)$ & $(5404)$ & $(5530)$ & $(4375)$ \\
\hline \multirow[t]{6}{*}{$T S$} & 30 & 0.1 & 5308 & 4990 & 4584 & 4747 & 5362 & 6117 & 7263 & 8087 & 5488 & 5298 & 5144 & 5548 \\
\hline & & & (7213) & (7508) & $(7008)$ & (7387) & $(6899)$ & $(7029)$ & $(7961)$ & $(8237)$ & $(7766)$ & (7936) & $(6567)$ & $(8052)$ \\
\hline & & 0.5 & 3057 & 3326 & 3732 & 4434 & 4785 & 4618 & 5877 & 7406 & 3855 & 4157 & 4036 & 3708 \\
\hline & & & (4216) & $(5215)$ & $(5687)$ & $(6652)$ & (5835) & $(4666)$ & $(5427)$ & $(6395)$ & $(4611)$ & $(5016)$ & $(5222)$ & $(4714)$ \\
\hline & & 0.9 & $\underline{2874}$ & 2987 & 3583 & 4353 & 4738 & 4560 & 6292 & 8495 & 3813 & 4056 & 3931 & 3570 \\
\hline & & & (3934) & $(4640)$ & $(5510)$ & $(6700)$ & (5585) & $(4569)$ & $(5859)$ & $(6950)$ & (4414) & $(4929)$ & (5309) & (4658) \\
\hline \multirow[t]{6}{*}{$J F$} & 2 & 0.1 & 5175 & 5307 & 4974 & 5014 & 5221 & 5587 & 6058 & 6421 & 5224 & 5804 & 5926 & 5267 \\
\hline & & & $(5740)$ & (7057) & $(6524)$ & (7018) & $(6112)$ & (6018) & $(5957)$ & (4998) & $(6320)$ & $(7074)$ & $(6431)$ & (6608) \\
\hline & & 0.5 & 3961 & 4195 & 4416 & 4610 & 4682 & 4554 & 5065 & 5614 & 4265 & 4964 & 5038 & 4150 \\
\hline & & & (4743) & $(5412)$ & $(6560)$ & $(6549)$ & $(5257)$ & $(4441)$ & $(4511)$ & (4498) & (4603) & $(5891)$ & $(6210)$ & $(4706)$ \\
\hline & & 0.9 & 3548 & 3887 & 4070 & 4506 & 4748 & 4155 & 4777 & 5468 & 3980 & 4656 & 4554 & 3817 \\
\hline & & & (4640) & $(5438)$ & $(5609)$ & $(6477)$ & $(5474)$ & $(4146)$ & $(4683)$ & $(4878)$ & $(4351)$ & $(5579)$ & $(5625)$ & $(4403)$ \\
\hline \multirow[t]{6}{*}{$J F$} & 30 & 0.1 & 6808 & 6348 & 6139 & 5570 & 5760 & 6905 & 7568 & 8463 & 6715 & 7146 & 7315 & 6572 \\
\hline & & & (6137) & $(5891)$ & $(6817)$ & (7097) & (5966) & $(6381)$ & $(5599)$ & $(6085)$ & (7364) & $(8813)$ & $(6553)$ & $(6831)$ \\
\hline & & 0.5 & 4303 & 4329 & 4393 & 4627 & 4554 & 4711 & 5400 & 6201 & 4540 & 4919 & 5137 & 4511 \\
\hline & & & (5092) & $(5433)$ & $(5794)$ & $(6618)$ & $(4591)$ & $(4243)$ & $(4661)$ & $(5190)$ & $(4965)$ & $(5559)$ & $(6004)$ & (5007) \\
\hline & & 0.9 & 3877 & 4000 & 4195 & 4439 & 4520 & 4579 & 5265 & 6262 & 4301 & 4689 & 4793 & 4257 \\
\hline & & & (4514) & $(5052)$ & $(5623)$ & $(6062)$ & $(5266)$ & $(4248)$ & $(4574)$ & $(5216)$ & $(4557)$ & $(5280)$ & $(5434)$ & $(4767)$ \\
\hline
\end{tabular}

The underlined or double-underlined number indicates the smallest or second smallest average ISE, respectively. 
Table 2: (continued).

\begin{tabular}{|c|c|c|c|c|c|c|c|c|c|c|c|c|c|c|}
\hline & $p$ & $a$ & $A_{2}$ & $A_{1.5}$ & $A_{1}$ & $A_{0.5}$ & $A_{-0.5}$ & $A_{-1}$ & $A_{-1.5}$ & $A_{-2}$ & $I G$ & $B S$ & $R I G$ & $L N_{-1 / 2}$ \\
\hline \multirow{2}{*}{\multicolumn{3}{|c|}{ original }} & 3726 & 3531 & 3222 & 2882 & 3084 & 3701 & 4098 & 4515 & 3470 & 3749 & 4063 & 3456 \\
\hline & & & $(2917)$ & $(3335)$ & $(3304)$ & $(2891)$ & $(2702)$ & (3209) & $(2766)$ & $(2800)$ & $(3329)$ & $(3342)$ & (3441) & (3299) \\
\hline \multirow[t]{6}{*}{$A D D$} & 2 & 0.1 & 2917 & 2798 & 2620 & 2502 & 2656 & 3020 & 3342 & 3711 & 2839 & 3083 & 3248 & 2792 \\
\hline & & & $(2771)$ & $(2849)$ & $(2843)$ & $(2772)$ & $(2581)$ & $(3253)$ & $(2720)$ & $(2883)$ & $(3334)$ & (3403) & $(3163)$ & (3223) \\
\hline & & 0.5 & 2601 & 2515 & 2342 & 2357 & 2482 & 2655 & 3028 & 3463 & 2476 & 2772 & 2948 & 2502 \\
\hline & & & (2983) & $(2955)$ & $(2724)$ & $(2720)$ & $(2472)$ & (2291) & $(2244)$ & $(2400)$ & $(2676)$ & (2931) & $(3172)$ & $(2786)$ \\
\hline & & 0.9 & 2464 & 2403 & 2321 & 2336 & 2477 & 2627 & 3010 & 3557 & 2430 & 2753 & 2893 & 2420 \\
\hline & & & $(2704)$ & $(2782)$ & $(2757)$ & $(2730)$ & $(2500)$ & $(2259)$ & $(2208)$ & $(2492)$ & $(2627)$ & $(3084)$ & $(3116)$ & $(2682)$ \\
\hline \multirow[t]{6}{*}{$A D D$} & 3 & 0.1 & 2859 & 2676 & 2488 & 2433 & 2591 & 3024 & 3448 & 3886 & 2750 & 2990 & 3161 & 2721 \\
\hline & & & (2899) & $(2870)$ & $(2855)$ & $(2804)$ & $(2673)$ & $(3426)$ & $(3219)$ & $(3471)$ & $(3431)$ & (3034) & (3043) & (3438) \\
\hline & & 0.5 & 2155 & 2195 & 2122 & 2240 & 2388 & 2398 & 2806 & 3350 & 2226 & 2501 & 2592 & 2209 \\
\hline & & & $(2666)$ & $(2975)$ & $(2729)$ & $(2663)$ & $(2476)$ & (2083) & $(2245)$ & $(2633)$ & $(2530)$ & $(2955)$ & $(3032)$ & $(2584)$ \\
\hline & & 0.9 & 2015 & 2075 & 2074 & 2206 & 2322 & 2318 & 2863 & 3425 & 2138 & 2414 & 2532 & 2125 \\
\hline & & & $(2362)$ & $(2889)$ & $(2786)$ & $(2633)$ & $(2416)$ & $(2079)$ & $(2311)$ & $(2560)$ & $(2483)$ & $(2902)$ & (3113) & (2509) \\
\hline \multirow[t]{6}{*}{$T S$} & 2 & 0.1 & 2566 & 2473 & 2398 & 2437 & 2553 & 2627 & 2742 & 2920 & 2544 & 2823 & 2880 & 2503 \\
\hline & & & $(2827)$ & $(2779)$ & (2799) & $(2720)$ & $(2414)$ & (3028) & $(2415)$ & (2331) & $(3289)$ & $(3418)$ & (3107) & (3209) \\
\hline & & 0.5 & 2095 & 2164 & 2137 & 2297 & 2445 & 2302 & 2600 & 2934 & 2199 & 2512 & 2624 & 2173 \\
\hline & & & $(2532)$ & $(2817)$ & $(2717)$ & $(2669)$ & $(2382)$ & (2090) & $(2273)$ & (2304) & $(2511)$ & (2907) & $(3070)$ & $(2612)$ \\
\hline & & 0.9 & 2015 & 2131 & 2103 & 2289 & 2443 & 2279 & 2602 & 3083 & 2191 & 2467 & 2592 & 2101 \\
\hline & & & $(2276)$ & $(2834)$ & $(2722)$ & $(2676)$ & $(2387)$ & (2035) & $(2153)$ & $(2376)$ & $(2548)$ & $(2862)$ & (3097) & $(2479)$ \\
\hline \multirow[t]{6}{*}{$T S$} & 3 & 0.1 & 2612 & 2436 & 2268 & 2332 & 2559 & 2979 & 3472 & 3954 & 2612 & 2666 & 2860 & 2621 \\
\hline & & & (3095) & $(3379)$ & $(2936)$ & $(2800)$ & $(2593)$ & (3508) & $(3455)$ & $(3781)$ & $(3435)$ & $(3288)$ & $(3531)$ & (3514) \\
\hline & & 0.5 & 1740 & 1824 & 1943 & 2212 & 2430 & 2328 & 3022 & 3909 & 2057 & 2299 & 2371 & 1965 \\
\hline & & & $(2207)$ & $(2660)$ & $(2761)$ & $(2664)$ & $(2382)$ & (2113) & $(2558)$ & $(3165)$ & $(2451)$ & $(2841)$ & $(3233)$ & $(2380)$ \\
\hline & & 0.9 & $\underline{1580}$ & $\underline{1633}$ & 1901 & 2193 & 2363 & 2320 & 3321 & 4510 & 2023 & 2191 & 2259 & 1862 \\
\hline & & & (1841) & $(2131)$ & $(2772)$ & $(2667)$ & $(2263)$ & (2034) & $(2739)$ & $(3380)$ & $(2408)$ & $(2727)$ & $(3040)$ & (2061) \\
\hline \multirow[t]{6}{*}{$J F$} & 2 & 0.1 & 2896 & 2778 & 2604 & 2493 & 2655 & 3022 & 3326 & 3680 & 2827 & 3046 & 3240 & 2773 \\
\hline & & & $(2777)$ & $(2850)$ & $(2829)$ & $(2767)$ & $(2582)$ & $(3286)$ & $(2738)$ & $(2862)$ & (3338) & (3389) & $(3201)$ & (3219) \\
\hline & & 0.5 & 2404 & 2355 & 2290 & 2318 & 2453 & 2541 & 2954 & 3344 & 2384 & 2694 & 2819 & 2341 \\
\hline & & & $(2710)$ & $(2878)$ & $(2822)$ & $(2717)$ & $(2432)$ & $(2270)$ & $(2335)$ & $(2453)$ & $(2656)$ & (2944) & $(3134)$ & $(2684)$ \\
\hline & & 0.9 & 2078 & 2171 & 2126 & 2291 & 2445 & 2321 & 2699 & 3148 & 2213 & 2527 & 2632 & 2156 \\
\hline & & & (2337) & $(2827)$ & $(2730)$ & $(2678)$ & (2397) & $(2027)$ & $(2162)$ & $(2351)$ & $(2502)$ & $(2862)$ & $(3075)$ & $(2514)$ \\
\hline \multirow[t]{6}{*}{$J F$} & 3 & 0.1 & 3492 & 3294 & 3035 & 2768 & 2937 & 3438 & 3869 & 4252 & 3252 & 3445 & 3750 & 3236 \\
\hline & & & $(2868)$ & $(2850)$ & $(2771)$ & $(2940)$ & $(2696)$ & (2779) & $(2915)$ & $(2872)$ & $(2887)$ & $(2804)$ & $(3116)$ & (2824) \\
\hline & & 0.5 & 2468 & 2360 & 2274 & 2289 & 2410 & 2584 & 2925 & 3356 & 2408 & 2654 & 2840 & 2420 \\
\hline & & & $(2790)$ & $(2856)$ & $(2785)$ & $(2742)$ & $(2548)$ & $(2237)$ & $(2286)$ & $(2446)$ & $(2675)$ & $(2973)$ & $(3197)$ & $(2727)$ \\
\hline & & 0.9 & 2240 & 2263 & 2187 & 2230 & 2346 & 2454 & 2925 & 3439 & 2295 & 2555 & 2699 & 2282 \\
\hline & & & $(2357)$ & (2939) & $(2782)$ & $(2637)$ & $(2537)$ & $(2017)$ & $(2306)$ & $(2536)$ & (2499) & (2987) & $(3044)$ & $(2526)$ \\
\hline
\end{tabular}


Table 3: Case C. The average ISEs $\times 10^{6}$ of estimators with/without bias corrections, where $A_{\gamma}, I G, B S, R I G$, and $L N_{-1 / 2}$ stand for the asymmetric KDEs (see Examples 1, 2, 3, and 5).

The number in the parentheses stands for the standard deviation $\times 10^{6}$ of the ISEs.

\begin{tabular}{|c|c|c|c|c|c|c|c|c|c|c|c|c|c|c|}
\hline & $p$ & $a$ & $A_{2}$ & $A_{1.5}$ & $A_{1}$ & $A_{0.5}$ & $A_{-0.5}$ & $A_{-1}$ & $A_{-1.5}$ & $A_{-2}$ & $I G$ & $B S$ & $R I G$ & $L N_{-1 / 2}$ \\
\hline \multicolumn{3}{|c|}{ original } & 7178 & 6656 & 6045 & 5411 & 5763 & 6688 & 7454 & 8060 & 6462 & 6926 & 7545 & 6482 \\
\hline & & & $(4902)$ & $(4722)$ & $(4604)$ & $(4325)$ & $(4565)$ & (4967) & $(4944)$ & $(4936)$ & $(4970)$ & $(5060)$ & $(5310)$ & (4912) \\
\hline \multirow[t]{6}{*}{$A D D$} & 2 & 0.1 & 7477 & 6978 & 6194 & 5339 & 5869 & 6845 & 7774 & 8532 & 6627 & 7148 & 7936 & 6707 \\
\hline & & & (4909) & $(4915)$ & $(4651)$ & $(4249)$ & $(4682)$ & $(4964)$ & $(4932)$ & $(4979)$ & $(4884)$ & $(5261)$ & $(5564)$ & $(4897)$ \\
\hline & & 0.5 & 7818 & 7199 & 6286 & 5335 & 5818 & 7126 & 8381 & 9492 & 6744 & 7294 & 8162 & 6814 \\
\hline & & & $(5031)$ & (4894) & $(4526)$ & $(4440)$ & $(4394)$ & $(4465)$ & $(4841)$ & $(5337)$ & $(4767)$ & $(5267)$ & $(5476)$ & (4653) \\
\hline & & 0.9 & 7788 & 7200 & 6309 & 5299 & 5871 & 7269 & 8489 & 9430 & 6769 & 7310 & 8144 & 6814 \\
\hline & & & $(4900)$ & (4911) & $(4600)$ & $(4395)$ & $(4562)$ & $(4511)$ & $(4977)$ & $(5501)$ & $(4667)$ & $(5238)$ & (5321) & (4677) \\
\hline \multirow[t]{6}{*}{$A D D$} & 3 & 0.1 & 7358 & 6711 & 5920 & $\underline{5164}$ & 5561 & 6482 & 7569 & 8409 & 6311 & 6995 & 7948 & 6369 \\
\hline & & & $(5172)$ & $(4992)$ & $(4618)$ & $(\overline{4128)}$ & $(4685)$ & $(5134)$ & $(5715)$ & $(5877)$ & $(5081)$ & $(5189)$ & $(5554)$ & $(5091)$ \\
\hline & & 0.5 & 8374 & 7636 & 6468 & 5301 & 5588 & 6191 & 7015 & 8125 & 6483 & 7503 & 8523 & 6620 \\
\hline & & & $(5196)$ & $(5102)$ & $(4652)$ & $(4386)$ & $(4253)$ & $(4423)$ & $(4727)$ & $(5347)$ & $(4598)$ & $(5307)$ & $(5645)$ & $(4709)$ \\
\hline & & 0.9 & 8534 & 7766 & 6495 & 5237 & 5600 & 6133 & 6967 & 7704 & 6527 & 7606 & 8770 & 6583 \\
\hline & & & $(4637)$ & $(5094)$ & $(4742)$ & $(4285)$ & $(4238)$ & $(4388)$ & $(4840)$ & $(5199)$ & $(4582)$ & $(5288)$ & $(5678)$ & (4587) \\
\hline \multirow[t]{6}{*}{$T S$} & 2 & 0.1 & 7898 & 7258 & 6314 & 5331 & 5891 & 7612 & 9214 & 10476 & 6882 & 7345 & 8292 & 6992 \\
\hline & & & $(5059)$ & (4934) & $(4687)$ & $(4287)$ & $(4530)$ & $(4595)$ & $(4846)$ & $(5234)$ & $(4659)$ & $(5119)$ & $(5531)$ & (4737) \\
\hline & & 0.5 & 8570 & 7694 & 6543 & 5303 & 5961 & 7884 & 9762 & 11405 & 7341 & 7537 & 8715 & 7681 \\
\hline & & & $(5066)$ & $(5186)$ & $(4768)$ & (4403) & $(4506)$ & $(4681)$ & $(5929)$ & $(7180)$ & $(4621)$ & $(5017)$ & $(5451)$ & (4491) \\
\hline & & 0.9 & 8522 & 7698 & 6582 & 5274 & 5797 & 6898 & 8032 & 9209 & 6972 & 7577 & 8757 & 7179 \\
\hline & & & $(4612)$ & $(5161)$ & $(4980)$ & $(4410)$ & $(4402)$ & $(4628)$ & $(5375)$ & (6409) & $(4654)$ & $(5075)$ & $(5430)$ & (4728) \\
\hline \multirow[t]{6}{*}{$T S$} & 3 & 0.1 & 7808 & 6964 & 6009 & $\underline{5117}$ & 5524 & 6566 & 7881 & 8975 & 6408 & 7103 & 8213 & 6491 \\
\hline & & & $(5548)$ & $(5183)$ & $(4798)$ & $(4154)$ & $(4735)$ & $(5264)$ & $(5942)$ & $(6215)$ & $(5214)$ & $(5329)$ & (5694) & $(5265)$ \\
\hline & & 0.5 & 8803 & 7906 & 6719 & 5274 & 5527 & 5981 & 6672 & 7750 & 6328 & 7667 & 9289 & 6484 \\
\hline & & & $(5285)$ & $(5011)$ & $(4807)$ & $(4447)$ & $(4431)$ & $(4881)$ & $(5048)$ & $(5534)$ & $(4828)$ & $(5196)$ & $(5335)$ & $(5092)$ \\
\hline & & 0.9 & 8880 & 8132 & 6809 & 5212 & 5430 & 5725 & 6468 & 7659 & 6291 & 7761 & 9706 & 6474 \\
\hline & & & $(4900)$ & $(5046)$ & $(5038)$ & (4319) & $(4166)$ & $(4412)$ & $(5121)$ & $(5914)$ & $(4647)$ & $(5221)$ & $(5382)$ & $(5156)$ \\
\hline \multirow[t]{6}{*}{$J F$} & 2 & 0.1 & 7493 & 6994 & 6202 & 5332 & 5872 & 6873 & 7797 & 8585 & 6645 & 7165 & 7966 & 6747 \\
\hline & & & $(4922)$ & (4913) & $(4652)$ & $(4254)$ & $(4727)$ & (4899) & $(4931)$ & $(4990)$ & $(4860)$ & $(5262)$ & (5583) & (5073) \\
\hline & & 0.5 & 8130 & 7402 & 6439 & 5313 & 5903 & 7698 & 9224 & 10554 & 6942 & 7437 & 8329 & 6986 \\
\hline & & & $(5080)$ & $(5080)$ & $(4748)$ & $(4396)$ & $(4431)$ & $(4377)$ & $(4948)$ & $(5869)$ & $(4652)$ & $(5257)$ & (5394) & $(4634)$ \\
\hline & & 0.9 & 8416 & 7628 & 6521 & 5283 & 5837 & 7051 & 8131 & 9201 & 7006 & 7525 & 8678 & 7182 \\
\hline & & & $(4791)$ & $(5136)$ & $(4763)$ & $(4421)$ & $(4440)$ & $(4656)$ & $(5287)$ & $(6011)$ & $(4645)$ & $(5060)$ & $(5417)$ & (4661) \\
\hline \multirow[t]{6}{*}{$J F$} & 3 & 0.1 & 7367 & 6837 & 6222 & 5370 & 5685 & 6874 & 7657 & 8392 & 6591 & 6950 & 7519 & 6633 \\
\hline & & & $(4911)$ & $(4754)$ & $(4617)$ & $(4227)$ & $(4240)$ & $(5031)$ & $(5011)$ & $(5431)$ & $(4805)$ & $(4819)$ & (4898) & (4808) \\
\hline & & 0.5 & 7739 & 7266 & 6365 & 5264 & 5770 & 6866 & 7692 & 8399 & 6741 & 7389 & 8264 & 6829 \\
\hline & & & $(4941)$ & $(4791)$ & $(4594)$ & $(4383)$ & $(4389)$ & $(4362)$ & $(4609)$ & $(4672)$ & (4499) & $(5161)$ & $(5440)$ & $(4560)$ \\
\hline & & 0.9 & 7797 & 7347 & 6425 & 5223 & 5792 & 6842 & 7576 & 8122 & 6845 & 7500 & 8471 & 6936 \\
\hline & & & $(4804)$ & $(5022)$ & $(4746)$ & $(4288)$ & $(4305)$ & $(4264)$ & $(4179)$ & $(4494)$ & $(4614)$ & $(5296)$ & $(5572)$ & (4503) \\
\hline
\end{tabular}

The underlined or double-underlined number indicates the smallest or second smallest average ISE, respectively. 
Table 3: (continued).

\begin{tabular}{|c|c|c|c|c|c|c|c|c|c|c|c|c|c|c|}
\hline & $p$ & $a$ & $A_{2}$ & $A_{1.5}$ & $A_{1}$ & $A_{0.5}$ & $A_{-0.5}$ & $A_{-1}$ & $A_{-1.5}$ & $A_{-2}$ & $I G$ & $B S$ & $R I G$ & $L N_{-1 / 2}$ \\
\hline \multirow{2}{*}{\multicolumn{3}{|c|}{ original }} & 4241 & 3911 & 3492 & 3048 & 3224 & 3774 & 4248 & 4696 & 3646 & 3977 & 4359 & 3681 \\
\hline & & & $(2773)$ & $(2770)$ & $(2659)$ & $(2492)$ & $(2634)$ & $(2799)$ & $(2842)$ & $(2942)$ & $(2777)$ & $(2813)$ & $(2895)$ & $(2802)$ \\
\hline \multirow[t]{6}{*}{$A D D$} & 2 & 0.1 & 4455 & 4042 & 3544 & 2933 & 3136 & 3779 & 4435 & 5108 & 3670 & 4055 & 4549 & 3701 \\
\hline & & & (2927) & $(2823)$ & $(2740)$ & $(2504)$ & $(2432)$ & $(2612)$ & $(3050)$ & (3308) & $(2657)$ & $(2887)$ & $(3032)$ & (2689) \\
\hline & & 0.5 & 4505 & 4049 & 3426 & 2835 & 3038 & 4127 & 5337 & 6288 & 3667 & 4058 & 4554 & 3695 \\
\hline & & & $(2852)$ & $(2712)$ & $(2322)$ & $(2041)$ & $(2202)$ & (3139) & $(3757)$ & $(4285)$ & $(2777)$ & (2893) & $(3076)$ & $(2816)$ \\
\hline & & 0.9 & 4472 & 3979 & 3424 & 2818 & 3023 & 4313 & 5776 & 6805 & 3651 & 4057 & 4526 & 3666 \\
\hline & & & $(2741)$ & $(2572)$ & $(2305)$ & (2018) & $(2200)$ & $(3153)$ & $(3886)$ & (4392) & (3011) & $(2917)$ & $(3064)$ & (2819) \\
\hline \multirow[t]{6}{*}{$A D D$} & 3 & 0.1 & 4466 & 4048 & 3542 & 2900 & 3083 & 3662 & 4271 & 4836 & 3599 & 4081 & 4578 & 3614 \\
\hline & & & $(2965)$ & $(2846)$ & $(2749)$ & $(2268)$ & $(2371)$ & $(2571)$ & $(2847)$ & (3189) & $(2587)$ & $(2902)$ & $(3048)$ & (2595) \\
\hline & & 0.5 & 4676 & 4095 & 3484 & 2816 & 3013 & 3594 & 4338 & 5097 & 3513 & 4077 & 4657 & 3591 \\
\hline & & & (3053) & $(2760)$ & $(2436)$ & $(2032)$ & $(2162)$ & (2621) & (3058) & $(3460)$ & $(2557)$ & $(2901)$ & $(3272)$ & (2739) \\
\hline & & 0.9 & 4877 & 4137 & 3433 & 2804 & 3035 & 3764 & 4633 & 5523 & 3593 & 4104 & 4760 & 3626 \\
\hline & & & $(3125)$ & $(2908)$ & $(2415)$ & $(2021)$ & $(2194)$ & $(2788)$ & $(3332)$ & $(4130)$ & $(2697)$ & $(2964)$ & $(3400)$ & $(2866)$ \\
\hline \multirow[t]{6}{*}{$T S$} & 2 & 0.1 & 4691 & 4169 & 3588 & 2894 & 3115 & 4307 & 5705 & 6884 & 3773 & 4168 & 4706 & 3805 \\
\hline & & & (3013) & $(2873)$ & $(2584)$ & $(2275)$ & $(2435)$ & $(3167)$ & $(3759)$ & (4343) & $(2745)$ & $(2988)$ & $(3120)$ & (2779) \\
\hline & & 0.5 & 4851 & 4234 & 3560 & 2816 & 2983 & 5476 & 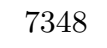 & 8759 & 4146 & 4187 & 4780 & 4273 \\
\hline & & & $(3053)$ & $(2842)$ & $(2471)$ & $(2019)$ & $(2127)$ & (3967) & $(5067)$ & (5958) & $(3164)$ & $(2836)$ & $(3179)$ & $(3257)$ \\
\hline & & 0.9 & 4791 & 4194 & 3546 & 2806 & 2995 & 4477 & 5837 & 6713 & 3942 & 4188 & 4825 & 4076 \\
\hline & & & $(2936)$ & $(2741)$ & $(2474)$ & $(2032)$ & $(2183)$ & $(3344)$ & $(4336)$ & $(4960)$ & $(3068)$ & $(2853)$ & $(3263)$ & (3196) \\
\hline \multirow[t]{6}{*}{$T S$} & 3 & 0.1 & 4651 & 4142 & 3522 & 2873 & 3052 & 3634 & 4308 & 4945 & 3586 & 4116 & 4734 & 3605 \\
\hline & & & (3083) & $(2902)$ & $(2471)$ & $(2253)$ & $(2358)$ & (2579) & $(2884)$ & $(3258)$ & $(2601)$ & $(2756)$ & $(3154)$ & (2616) \\
\hline & & 0.5 & 4948 & 4297 & 3560 & 2803 & 2946 & 3459 & 4065 & 4667 & 3541 & 4234 & 5141 & 3579 \\
\hline & & & $(3240)$ & $(2845)$ & (2409) & $(2026)$ & $(2074)$ & (2499) & $(2923)$ & (3188) & $(2591)$ & $(2938)$ & $(3497)$ & $(2780)$ \\
\hline & & 0.9 & 5367 & 4335 & 3548 & $\underline{2792}$ & 2979 & 3499 & 4109 & 4943 & 3509 & 4346 & 5557 & 3520 \\
\hline & & & $(3543)$ & (2918) & $(2440)$ & $(2003)$ & $(2131)$ & $(2480)$ & $(3061)$ & $(4306)$ & $(2511)$ & $(3068)$ & $(3774)$ & (2551) \\
\hline \multirow[t]{6}{*}{$J F$} & 2 & 0.1 & 4471 & 4049 & 3550 & 2932 & 3132 & 3794 & 4449 & 5139 & 3677 & 4073 & 4561 & 3694 \\
\hline & & & (2923) & $(2827)$ & $(2748)$ & $(2512)$ & $(2433)$ & $(2634)$ & (3048) & (3338) & $(2662)$ & $(2920)$ & $(3060)$ & $(2670)$ \\
\hline & & 0.5 & 4688 & 4147 & 3450 & 2821 & 3036 & 4601 & 6325 & 7562 & 3744 & 4126 & 4635 & 3773 \\
\hline & & & (3021) & $(3003)$ & $(2278)$ & (2031) & $(2237)$ & (3395) & $(4163)$ & $(4860)$ & $(2844)$ & $(2917)$ & $(3086)$ & $(2892)$ \\
\hline & & 0.9 & 4730 & 4156 & 3525 & $\underline{\underline{2802}}$ & 3006 & 4596 & 6092 & 6985 & 3948 & 4179 & 4759 & 4063 \\
\hline & & & $(2821)$ & $(2642)$ & $(2382)$ & $(2000)$ & $(2194)$ & (3391) & $(4346)$ & $(4864)$ & $(3075)$ & $(2854)$ & $(3207)$ & (3169) \\
\hline \multirow[t]{6}{*}{$J F$} & 3 & 0.1 & 4214 & 3921 & 3511 & 2959 & 3158 & 3781 & 4290 & 4676 & 3649 & 3955 & 4323 & 3664 \\
\hline & & & $(2922)$ & (2919) & $(2812)$ & $(2592)$ & $(2704)$ & (2709) & $(2954)$ & (2881) & $(2680)$ & $(2914)$ & $(3036)$ & $(2685)$ \\
\hline & & 0.5 & 4431 & 4030 & 3444 & 2807 & 3031 & 3908 & 4639 & 5127 & 3577 & 4074 & 4561 & 3625 \\
\hline & & & $(2859)$ & $(2741)$ & $(2364)$ & $(2037)$ & $(2214)$ & (2910) & $(3279)$ & $(3372)$ & $(2565)$ & $(2968)$ & $(3134)$ & $(2681)$ \\
\hline & & 0.9 & 4414 & 3994 & 3472 & 2809 & 3064 & 4211 & 4984 & 5425 & 3656 & 4077 & 4656 & 3774 \\
\hline & & & $(2877)$ & $(2692)$ & $(2665)$ & $(2030)$ & $(2284)$ & $(3016)$ & $(3257)$ & $(3365)$ & (2687) & (3013) & (3301) & $(3000)$ \\
\hline
\end{tabular}


Table 4: Case D. The average ISEs $\times 10^{6}$ of estimators with/without bias corrections, where $A_{\gamma}, I G, B S, R I G$, and $L N_{-1 / 2}$ stand for the asymmetric KDEs (see Examples 1, 2, 3, and 5).

The number in the parentheses stands for the standard deviation $\times 10^{6}$ of the ISEs.

\begin{tabular}{|c|c|c|c|c|c|c|c|c|c|c|c|c|c|c|}
\hline & $p$ & $a$ & $A_{2}$ & $A_{1.5}$ & $A_{1}$ & $A_{0.5}$ & $A_{-0.5}$ & $A_{-1}$ & $A_{-1.5}$ & $A_{-2}$ & $I G$ & $B S$ & $R I G$ & $L N_{-1 / 2}$ \\
\hline \multicolumn{3}{|c|}{ original } & 4665 & 4230 & 3669 & 3168 & 3155 & 3800 & 4600 & 5371 & 3708 & 4170 & 4705 & 3721 \\
\hline & & & $(2851)$ & $(2744)$ & $(2491)$ & $(2295)$ & $(2316)$ & (2659) & (3011) & $(3326)$ & (2599) & $(2736)$ & $(2907)$ & $(2586)$ \\
\hline \multirow[t]{6}{*}{$A D D$} & 2 & 0.1 & 5011 & 4399 & 3736 & 3190 & 3120 & 4095 & 5443 & 6363 & 3699 & 4223 & 4890 & 3786 \\
\hline & & & $(3046)$ & $(2861)$ & $(2592)$ & $(2436)$ & $(2405)$ & $(3053)$ & $(3537)$ & $(3660)$ & $(2681)$ & $(2826)$ & $(3113)$ & $(2742)$ \\
\hline & & 0.5 & 5191 & 4602 & 3818 & 3159 & 3046 & 4362 & 5559 & 6291 & 3763 & 4257 & 5065 & 3940 \\
\hline & & & $(3076)$ & (3013) & $(2689)$ & $(2400)$ & $(2372)$ & (3204) & $(3318)$ & $(3280)$ & (2895) & (2967) & $(3359)$ & $(3065)$ \\
\hline & & 0.9 & 5209 & 4627 & 3848 & 3151 & 3071 & 3881 & 4744 & 5299 & 3662 & 4247 & 5103 & 3783 \\
\hline & & & $(2817)$ & (3021) & $(2751)$ & $(2350)$ & $(2460)$ & (2977) & $(3083)$ & $(3130)$ & (2795) & $(2970)$ & $(3430)$ & (2898) \\
\hline \multirow[t]{6}{*}{$A D D$} & 3 & 0.1 & 4861 & 4301 & 3625 & 3129 & 3099 & 3702 & 4466 & 5138 & 3644 & 4180 & 4847 & 3681 \\
\hline & & & $(3227)$ & $(2952)$ & $(2612)$ & $(2403)$ & $(2414)$ & (2698) & $(2997)$ & $(3230)$ & $(2679)$ & $(2827)$ & (3109) & $(2691)$ \\
\hline & & 0.5 & 5342 & 4708 & 3879 & 3146 & $\underline{3023}$ & 3442 & 4189 & 4861 & 3522 & 4217 & 5174 & 3585 \\
\hline & & & $(3145)$ & (3087) & $(2796)$ & $(2267)$ & $(2397)$ & (2648) & $(2996)$ & $(3208)$ & $(2679)$ & $(2984)$ & $(3530)$ & $(2744)$ \\
\hline & & 0.9 & 5254 & 4766 & 3864 & 3155 & $\underline{2984}$ & 3422 & 4111 & 4800 & 3497 & 4243 & 5289 & 3561 \\
\hline & & & $(2751)$ & (3094) & $(2729)$ & $(2294)$ & $(2276)$ & $(2684)$ & $(3016)$ & $(3238)$ & $(2694)$ & $(3021)$ & $(3650)$ & $(2722)$ \\
\hline \multirow[t]{6}{*}{$T S$} & 2 & 0.1 & 5265 & 4583 & 3834 & 3233 & 3171 & 4589 & 5628 & 6328 & 4008 & 4368 & 5168 & 4211 \\
\hline & & & $(3144)$ & $(2986)$ & $(2613)$ & $(2392)$ & $(2409)$ & $(3346)$ & $(3790)$ & $(4061)$ & $(2972)$ & $(2901)$ & $(3277)$ & (3157) \\
\hline & & 0.5 & 5446 & 4875 & 4000 & 3240 & 3177 & 4241 & 5285 & 6080 & 3906 & 4572 & 5616 & 4052 \\
\hline & & & $(2984)$ & (3064) & $(2711)$ & $(2371)$ & $(2464)$ & (2984) & $(3158)$ & $(3268)$ & $(2839)$ & $(3079)$ & $(3649)$ & (2988) \\
\hline & & 0.9 & 5424 & 4854 & 4014 & 3236 & 3152 & 4004 & 4794 & 5312 & 3822 & 4541 & 5552 & 3922 \\
\hline & & & $(2876)$ & (2987) & $(2702)$ & $(2341)$ & $(2382)$ & (2969) & (3013) & $(3071)$ & $(2718)$ & (3019) & $(3559)$ & $(2783)$ \\
\hline \multirow[t]{6}{*}{$T S$} & 3 & 0.1 & 5108 & 4493 & 3740 & 3183 & 3153 & 3804 & 4624 & 5372 & 3753 & 4313 & 5020 & 3795 \\
\hline & & & $(3315)$ & $(3073)$ & $(2689)$ & $(2422)$ & $(2414)$ & $(2724)$ & $(3035)$ & $(3292)$ & $(2711)$ & $(2887)$ & $(3149)$ & $(2722)$ \\
\hline & & 0.5 & 5641 & 5008 & 4079 & 3284 & 3167 & 3692 & 4381 & 5003 & 3817 & 4537 & 5444 & 3853 \\
\hline & & & (3108) & (3114) & $(2751)$ & $(2394)$ & $(2420)$ & (2666) & $(2997)$ & $(3180)$ & $(2728)$ & $(3017)$ & $(3423)$ & $(2727)$ \\
\hline & & 0.9 & 5489 & 5033 & 4134 & 3273 & 3128 & 3675 & 4278 & 4838 & 3816 & 4587 & 5584 & 3882 \\
\hline & & & $(2642)$ & $(3024)$ & $(2755)$ & $(2353)$ & $(2279)$ & (2689) & $(3004)$ & $(3161)$ & $(2716)$ & $(3034)$ & $(3547)$ & $(2782)$ \\
\hline \multirow[t]{6}{*}{$J F$} & 2 & 0.1 & 5046 & 4415 & 3747 & 3196 & 3126 & 4139 & 5510 & 6479 & 3718 & 4240 & 4919 & 3804 \\
\hline & & & $(3086)$ & $(2864)$ & $(2593)$ & $(2442)$ & $(2405)$ & (3048) & $(3571)$ & (3699) & (2695) & $(2831)$ & $(3124)$ & $(2747)$ \\
\hline & & 0.5 & 5328 & 4751 & 3910 & 3200 & 3103 & 4353 & 5429 & 6107 & 3860 & 4403 & 5263 & 4047 \\
\hline & & & $(2960)$ & $(3006)$ & $(2678)$ & $(2395)$ & $(2386)$ & (3103) & $(3208)$ & $(3170)$ & (2902) & $(2979)$ & $(3394)$ & $(3060)$ \\
\hline & & 0.9 & 5402 & 4836 & 3997 & 3227 & 3144 & 3979 & 4774 & 5282 & 3805 & 4502 & 5534 & 3907 \\
\hline & & & $(2852)$ & (2987) & $(2701)$ & $(2340)$ & $(2381)$ & $(2946)$ & $(3007)$ & $(3054)$ & $(2718)$ & $(2992)$ & $(3561)$ & (2791) \\
\hline \multirow[t]{6}{*}{$J F$} & 3 & 0.1 & 4554 & 4102 & 3588 & 3155 & 3222 & 3852 & 4414 & 4855 & 3730 & 4141 & 4621 & 3757 \\
\hline & & & $(2874)$ & $(2727)$ & $(2548)$ & $(2343)$ & $(2374)$ & (2693) & $(2837)$ & (3027) & $(2640)$ & $(2748)$ & $(2893)$ & (2644) \\
\hline & & 0.5 & 5366 & 4751 & 3938 & 3151 & 3082 & 3590 & 4253 & 4887 & 3665 & 4347 & 5235 & 3738 \\
\hline & & & $(3034)$ & $(3007)$ & $(2739)$ & $(2235)$ & $(2394)$ & $(2716)$ & $(2961)$ & $(3183)$ & $(2704)$ & (2999) & $(3402)$ & $(2758)$ \\
\hline & & 0.9 & 5326 & 4819 & 3933 & 3153 & 3034 & 3521 & 4121 & 4706 & 3622 & 4371 & 5370 & 3698 \\
\hline & & & $(2683)$ & $(2972)$ & $(2731)$ & (2209) & $(2266)$ & $(2710)$ & $(2974)$ & $(3088)$ & $(2706)$ & $(3040)$ & $(3553)$ & (2794) \\
\hline
\end{tabular}

The underlined or double-underlined number indicates the smallest or second smallest average ISE, respectively. 
Table 4: (continued).

\begin{tabular}{|c|c|c|c|c|c|c|c|c|c|c|c|c|c|c|}
\hline & $p$ & $a$ & $A_{2}$ & $A_{1.5}$ & $A_{1}$ & $A_{0.5}$ & $A_{-0.5}$ & $A_{-1}$ & $A_{-1.5}$ & $A_{-2}$ & $I G$ & $B S$ & $R I G$ & $L N_{-1 / 2}$ \\
\hline \multirow{2}{*}{\multicolumn{3}{|c|}{ original }} & 2896 & 2615 & 2286 & 1988 & 1976 & 2311 & 2683 & 3055 & 2287 & 2579 & 2874 & 2293 \\
\hline & & & $(1845)$ & $(1780)$ & (1677) & $(1564)$ & $(1548)$ & (1724) & $(1828)$ & (2007) & $(1714)$ & $(1775)$ & (1851) & (1717) \\
\hline \multirow[t]{6}{*}{$A D D$} & 2 & 0.1 & 3016 & 2677 & 2273 & 1975 & 1924 & 2240 & 2870 & 3772 & 2195 & 2568 & 2913 & 2222 \\
\hline & & & (1957) & (1908) & $(1762)$ & (1633) & (1593) & (1792) & $(2245)$ & (2902) & $(1726)$ & $(1862)$ & (1963) & (1757) \\
\hline & & 0.5 & 3155 & 2711 & 2273 & 1963 & 1891 & 2424 & 3998 & 5128 & 2136 & 2509 & 2971 & 2159 \\
\hline & & & (2089) & (1988) & $(1775)$ & (1624) & (1593) & $(2281)$ & $(2988)$ & (3031) & $(1732)$ & (1869) & $(2127)$ & (1811) \\
\hline & & 0.9 & 3175 & 2715 & 2257 & 1952 & $\underline{1877}$ & 2155 & 3056 & 3774 & 2145 & 2521 & 2948 & 2140 \\
\hline & & & (1950) & (1986) & $(1754)$ & (1610) & (1593) & (1861) & $(2492)$ & $(2542)$ & $(1755)$ & (1890) & $(2103)$ & (1754) \\
\hline \multirow[t]{6}{*}{$A D D$} & 3 & 0.1 & 3031 & 2693 & 2269 & 1968 & 1924 & 2222 & 2645 & 3034 & 2207 & 2567 & 2927 & 2226 \\
\hline & & & (2028) & (1930) & (1759) & (1613) & $(1685)$ & (1771) & $(1895)$ & (1998) & $(1745)$ & (1868) & (1983) & (1766) \\
\hline & & 0.5 & 3319 & 2834 & 2318 & 1944 & 1888 & 2038 & 2450 & 2908 & 2107 & 2552 & 2981 & 2133 \\
\hline & & & (2063) & $(2067)$ & $(1860)$ & (1389) & (1631) & (1746) & (1941) & (2109) & $(1751)$ & (1982) & $(2167)$ & (1802) \\
\hline & & 0.9 & 3604 & 2876 & 2320 & 1945 & $\underline{1883}$ & 2023 & 2439 & 3022 & 2109 & 2573 & 3014 & 2098 \\
\hline & & & (1851) & $(2135)$ & (1856) & (1388) & (1626) & (1740) & $(1795)$ & $(2351)$ & $(1785)$ & (2013) & $(2233)$ & (1764) \\
\hline \multirow[t]{6}{*}{$T S$} & 2 & 0.1 & 3131 & 2771 & 2352 & 2012 & 1964 & 2590 & 3413 & 4009 & 2295 & 2667 & 3036 & 2329 \\
\hline & & & (2028) & (1919) & (1777) & (1638) & (1598) & $(2297)$ & $(2900)$ & $(3286)$ & (1779) & (1886) & (1985) & (1814) \\
\hline & & 0.5 & 3376 & 2912 & 2404 & 2020 & 1945 & 2536 & 3863 & 4928 & 228 & 2722 & 3191 & 2308 \\
\hline & & & (2081) & (2017) & $(1810)$ & (1635) & $(1596)$ & (2173) & $(2745)$ & $(2872)$ & $(1771)$ & (1937) & $(2152)$ & (1842) \\
\hline & & 0.9 & 3385 & 2914 & 2416 & 2010 & 1935 & 2281 & 3079 & 3780 & 2289 & 2729 & 3205 & 2285 \\
\hline & & & (1919) & $(2014)$ & (1809) & $(1610)$ & $(1587)$ & (1812) & $(2319)$ & (2411) & (1790) & (1919) & $(2162)$ & (1793) \\
\hline \multirow[t]{6}{*}{$T S$} & 3 & 0.1 & 3156 & 2795 & 2350 & 2002 & 1962 & 2314 & 2758 & 3164 & 2295 & 2681 & 3046 & 2313 \\
\hline & & & (2048) & (1940) & (1769) & (1603) & (1601) & (1793) & (1911) & (2025) & $(1761)$ & (1904) & (1994) & (1782) \\
\hline & & 0.5 & 3565 & 3042 & 2471 & 2029 & 1953 & 2229 & 2643 & 3060 & 2299 & 2777 & 3281 & 2309 \\
\hline & & & (1989) & $(2068)$ & (1851) & (1636) & (1636) & (1809) & $(1972)$ & $(2100)$ & (1793) & (1998) & $(2197)$ & (1848) \\
\hline & & 0.9 & 3790 & 3103 & 2505 & 2017 & 1946 & 2216 & 2677 & 3140 & 2317 & 2807 & 3303 & 2305 \\
\hline & & & (1717) & $(2048)$ & (1854) & (1441) & (1628) & (1801) & $(2005)$ & $(2266)$ & $(1805)$ & (1992) & $(2200)$ & (1821) \\
\hline \multirow[t]{6}{*}{$J F$} & 2 & 0.1 & 3035 & 2693 & 2282 & 1979 & 1930 & 2266 & 2926 & 3860 & 2219 & 2582 & 2929 & 2238 \\
\hline & & & (2010) & (1910) & $(1762)$ & $(1634)$ & $(1592)$ & (1810) & $(2297)$ & $(2950)$ & $(1741)$ & (1863) & (1962) & (1756) \\
\hline & & 0.5 & 3282 & 2833 & 2347 & 1997 & 1917 & 2499 & 3942 & 5001 & 2223 & 2636 & 3091 & 2229 \\
\hline & & & (2094) & (2009) & $(1805)$ & (1637) & (1594) & $(2229)$ & $(2815)$ & $(2852)$ & (1771) & (1914) & $(2106)$ & (1805) \\
\hline & & 0.9 & 3359 & 2900 & 2394 & 2003 & 1937 & 2269 & 3100 & 3762 & 2279 & 2718 & 3175 & 2283 \\
\hline & & & (1905) & $(2012)$ & $(1784)$ & (1608) & (1604) & (1814) & $(2357)$ & (2399) & (1790) & (1920) & $(2134)$ & (1814) \\
\hline \multirow[t]{6}{*}{$J F$} & 3 & 0.1 & 2957 & 2693 & 2347 & 2009 & 2029 & 2433 & 2817 & 3101 & 2389 & 2666 & 2955 & 2402 \\
\hline & & & (1869) & (1796) & $(1682)$ & $(1521)$ & $(1525)$ & (1730) & $(1842)$ & (1907) & (1703) & (1803) & (1881) & (1706) \\
\hline & & 0.5 & 3338 & 2889 & 2357 & 1997 & 1922 & 2147 & 2514 & 2960 & 2208 & 2633 & 3088 & 2218 \\
\hline & & & (1959) & (2037) & (1788) & $(1640)$ & $(1615)$ & (1805) & $(1922)$ & $(2146)$ & $(1752)$ & (1934) & $(2132)$ & (1793) \\
\hline & & 0.9 & 3658 & 2930 & 2394 & 1960 & 1919 & 2085 & 2503 & 3031 & 2189 & 2659 & 3105 & 2178 \\
\hline & & & (1806) & $(2096)$ & (1858) & (1390) & (1653) & $(1725)$ & $(1862)$ & $(2286)$ & (1767) & (1988) & $(2193)$ & (1783) \\
\hline
\end{tabular}


be constructed, where $K_{[2 p], \boldsymbol{a}}(\cdot)=\sum_{j=1}^{p} a_{j} c_{j}\left(\boldsymbol{a}^{2}\right) K_{[2]}\left(a_{j} \cdot\right)$ is a $2 p$ th-order kernel, that is an extension of Schucany and Sommers' fourth-order kernel $K_{[4],(1, a)}(a \neq 1)$, as mentioned in Introduction. Such kernels (independent of interest) form a class of $2 p$ th-order kernels, whose limiting version may be also considered. For example, we produce a class of 6th-order kernels from a given $K_{[2]}$, as follows:

$$
K_{[6],(1, a, 1 / a)}(s)=\left\{\begin{array}{lr}
\frac{1}{\left(a^{2}+1\right)\left(a^{2}-1\right)^{2}}\left\{-a^{2}\left(a^{2}+1\right) K_{[2]}(s)+a^{7} K_{[2]}(a s)+\frac{1}{a} K_{[2]}\left(\frac{s}{a}\right)\right\}, & a \neq 1, \\
\frac{1}{8}\left\{15 K_{[2]}(s)+9 s K_{[2]}^{\prime}(s)+s^{2} K_{[2]}^{\prime \prime}(s)\right\}, & a=1 .
\end{array}\right.
$$

Setting $K_{[2]}(s)=e^{-s^{2} / 2} / \sqrt{2 \pi}=\phi(s)$ (say), we obtain the (Gaussian-based) 6th-order kernel $\phi(s)\left(15-10 s^{2}+s^{4}\right) / 8$, which is found in Nadaraya (1974) and Wand and Schucany (1990). As alternatives to the additive-type bias-corrected estimator; $\sum_{j=1}^{p} c_{j}\left(a_{1}^{2}, \ldots, a_{p}^{2}\right) \widehat{f}_{h / a_{j}}^{\left(K_{[2]}\right)}(x)$, the TS/JF-type bias-corrected estimators can be further proposed (the details are omitted).

Remark 8 In Terrell and Scott (1980), a linear combination of the Rosenblatt-Parzen KDEs; $\sum_{j=1}^{p} c_{j}\left(1,1 / 2^{2}, \ldots, 1 / p^{2}\right) \hat{f}_{j h}^{\left(K_{[2]}\right)}(x)$, as well as a multiplicative analogue (they are what we call the additive-type and TS-type bias correction methods), were already mentioned to obtain the faster convergence rate of the MISE; $n^{-4 p /(4 p+1)}$, where

$$
c_{j}\left(1,1 / 2^{2}, \ldots, 1 / p^{2}\right)=2(-1)^{j-1} \frac{\prod_{i=1}^{j}(p-i+1)}{\prod_{i=1}^{j}(p+i)} \quad \text { for } j=1, \ldots, p .
$$

\subsection{Multivariate density estimation}

We briefly discuss the extension of the bias correction methods to the multivariate setting with $\operatorname{supp}(f)=[0, \infty)^{m}$ (the case $[0,1]^{m}$ is similar ${ }^{[8]}$. Let $\widehat{f}_{\beta}(\boldsymbol{x}), \boldsymbol{x}=\left(x_{1}, \ldots, x_{m}\right)^{\prime} \in[0, \infty)^{m}$, be a density estimator, such that $E\left[\widehat{f}_{\beta}(\boldsymbol{x})\right]=f(\boldsymbol{x})+\sum_{j=1}^{p} \beta^{j} \gamma_{j, m}(\boldsymbol{x} ; f)+o\left(\beta^{p}\right)$ for some $p \in \mathbb{N} \backslash\{1\}$ and functions $\gamma_{j, m}(\cdot ; f), j=1, \ldots, p$, independent of $\beta$. Such an estimator can be constructed using the product kernel, as follows: for a random sample $\left\{\boldsymbol{X}_{i}=\left(X_{i 1}, \ldots, X_{i m}\right)^{\prime}, i=1, \ldots, n\right\}$ of size $n, \widehat{f}_{\beta}(\boldsymbol{x})=n^{-1} \sum_{i=1}^{n} \prod_{j=1}^{m} K\left(X_{i j}, x_{j}, c_{j} \beta\right)$ is a product-type asymmetric KDE, where $c_{j}$ 's are positive constants, independent of $\beta$ and $\boldsymbol{x}$. Given a positive vector $\boldsymbol{a}=\left(a_{1}, \ldots, a_{p}\right)^{\prime}$, such that the $a_{k}$ 's are distinct, the bias-corrected estimators are defined by

$$
\begin{aligned}
\widehat{f}_{\beta, A D D_{a}^{p}}(\boldsymbol{x}) & =\sum_{k=1}^{p} c_{k}(\boldsymbol{a}) \widehat{f}_{\beta / a_{k}}(\boldsymbol{x}), \quad \widehat{f}_{\beta, T S_{a}^{p}}(\boldsymbol{x})=\prod_{k=1}^{p}\left\{\widehat{f}_{\beta / a_{k}}(\boldsymbol{x})+\frac{\epsilon}{a_{k}}\right\}^{c_{k}(\boldsymbol{a})}, \\
\widehat{f}_{\beta, J F_{\boldsymbol{a}}^{p}}(\boldsymbol{x}) & =\left\{\widehat{f}_{\beta}(\boldsymbol{x})+\epsilon\right\} \exp \left[\sum_{j=1}^{p-1} \frac{(-1)^{j-1}}{j}\left\{\frac{\widehat{f}_{\beta, A D D_{a}^{p}}(\boldsymbol{x})}{\widehat{f}_{\beta}(\boldsymbol{x})+\epsilon}-1\right\}^{j}\right] .
\end{aligned}
$$

The details are omitted here.

\footnotetext{
${ }^{[8]}$ We do not pursue the density estimation with mixed support, such as $\operatorname{supp}(f)=[0, \infty) \times \mathbb{R}$.
} 


\section{Appendix A.1. Preliminary results}

We write

$$
\bar{\Delta}_{\beta}(x)=\widehat{f}_{\beta}(x)-E\left[\widehat{f}_{\beta}(x)\right],
$$

which is the average of zero-mean independent random variables

$$
\Delta\left(X_{i} ; x, \beta\right)=K\left(X_{i} ; x, \beta\right)-E\left[K\left(X_{i} ; x, \beta\right)\right], \quad i=1, \ldots, n .
$$

Note that $V\left[\widehat{f}_{\beta}(x)\right]=V\left[\bar{\Delta}_{\beta}(x)\right]=n^{-1} E\left[\Delta^{2}\left(X_{1} ; x, \beta\right)\right]$. Also, we write

$$
\bar{\Delta}_{\beta, A D D_{\boldsymbol{a}}^{p}}(x)=\sum_{k=1}^{p} c_{k}(\boldsymbol{a}) \bar{\Delta}_{\beta / a_{k}}(x)=\widehat{f}_{\beta, A D D_{\boldsymbol{a}}^{p}}(x)-E\left[\widehat{f}_{\beta, A D D_{\boldsymbol{a}}^{p}}(x)\right],
$$

which is the average of zero-mean independent random variables

$$
\Delta_{A D D_{\boldsymbol{a}}^{p}}\left(X_{i} ; x, \beta\right)=\sum_{k=1}^{p} c_{k}(\boldsymbol{a}) \Delta\left(X_{i} ; x, \beta / a_{k}\right), \quad i=1, \ldots, n .
$$

Lemma A.1 Suppose that Assumption A1(i) holds. For any $n \in \mathbb{N}, \beta, t>0, x \in \mathcal{S}$, and $j \geq 2$, we have

(i) $E\left[\left|\bar{\Delta}_{\beta}(x)\right|^{j}\right] \leq C(j)\left\{\frac{1}{n^{j-1}} E\left[\left|\Delta\left(X_{1} ; x, \beta\right)\right|^{j}\right]+\left(\frac{1}{n} E\left[\Delta^{2}\left(X_{1} ; x, \beta\right)\right]\right)^{j / 2}\right\}$

(the constant $C(j)>0$ depends only on $j$ )

$$
\begin{aligned}
& \leq C(j)\left\{\left(\frac{C_{K} \beta^{-1}}{n}\right)^{j-2}+\left(\frac{C_{K} \beta^{-1}}{n}\right)^{(j-2) / 2}\right\} \frac{1}{n} E\left[\Delta^{2}\left(X_{1} ; x, \beta\right)\right] \\
& =O\left((n \beta)^{-(j-2) / 2} V\left[\widehat{f}_{\beta}(x)\right]\right) \quad(\text { if } j>2, \text { assume } n \beta \rightarrow \infty),
\end{aligned}
$$

(ii) $P\left[\left|\bar{\Delta}_{\beta}(x)\right| \geq t\right] \leq 2 \exp \left\{-\frac{n \beta t^{2}}{C_{K}\left(2\|f\|_{\mathcal{S}}+t\right)}\right\}$,

(iii) $P\left[\left|\bar{\Delta}_{\beta, A D D_{a}^{p}}(x)\right| \geq t\right] \leq 2 \exp \left[-\frac{n \beta t^{2}}{C_{K}\left\{2 p \sum_{k=1}^{p} c_{k}^{2}(\boldsymbol{a}) a_{k}|| f \|_{\mathcal{S}}+t \sum_{k=1}^{p}\left|c_{k}(\boldsymbol{a})\right| a_{k}\right\}}\right]$.

Proof Assumption A1(i) enables us to see that, for $i=1, \ldots, n$,

$$
\begin{aligned}
\left|\Delta\left(X_{i} ; x, \beta\right)\right| & \leq \sup _{s \in \mathcal{S}} K(s ; x, \beta) \leq C_{K} \beta^{-1}, \\
V\left[\Delta\left(X_{i} ; x, \beta\right)\right] & \leq \int_{\mathcal{S}} K^{2}(s ; x, \beta) f(s) d s \leq C_{K} \beta^{-1}\|f\|_{\mathcal{S} .}
\end{aligned}
$$

Hence, Rosenthal's inequality and Bennett's inequality yield the results (i) and (ii). Similarly, we have the result (iii), noting that

$$
\begin{aligned}
&\left|\Delta_{A D D_{\boldsymbol{a}}^{p}}\left(X_{i} ; x, \beta\right)\right| \leq \sum_{k=1}^{p}\left|c_{k}(\boldsymbol{a})\right|\left|\Delta\left(X_{i} ; x, \beta / a_{k}\right)\right| \leq \sum_{k=1}^{p}\left|c_{k}(\boldsymbol{a})\right| C_{K} a_{k} \beta^{-1}, \\
& V\left[\Delta_{A D D_{\boldsymbol{a}}^{p}}\left(X_{i} ; x, \beta\right)\right] \leq p \sum_{k=1}^{p} c_{k}^{2}(\boldsymbol{a}) V\left[\Delta\left(X_{i} ; x, \beta / a_{k}\right)\right] \leq p \sum_{k=1}^{p} c_{k}^{2}(\boldsymbol{a}) C_{K} a_{k} \beta^{-1}\|f\|_{\mathcal{S}} .
\end{aligned}
$$


Lemma A.2 Let $a_{0}, a_{0}^{\prime}>0$ be arbitrary constants.

(i) Suppose that Assumptions A1, A2, A4'[1], and A5'(i) hold. Then, $\operatorname{Cov}\left[\widehat{f}_{\beta / a_{0}}(x), \widehat{f}_{\beta / a_{0}^{\prime}}(x)\right]= \begin{cases}n^{-1} \beta^{-1 / 2}\left(\frac{2 a_{0} a_{0}^{\prime}}{a_{0}+a_{0}^{\prime}}\right)^{1 / 2} V(x ; f)\left[1+O\left(\beta \psi^{-1}(x)\right)\right]+O\left(n^{-1}\right), & x \in \mathcal{S}_{I, \beta}, \\ n^{-1} \beta^{-1} f(x)\left[\varsigma_{a_{0}, a_{0}^{\prime}}(\kappa)+\chi_{\left\{x \notin \mathcal{S}_{B}\right\}} O(1)\right]+O\left(n^{-1}\right), & x \in \mathcal{S}_{B, \beta, \kappa} .\end{cases}$

(ii) Suppose that Assumptions A1-A3, A4'[1], and $\mathrm{A} 5^{\prime}$ hold. Then,

$$
\int_{\mathcal{S}} \operatorname{Cov}\left[\widehat{f}_{\beta / a_{0}}(x), \widehat{f}_{\beta / a_{0}^{\prime}}(x)\right] d x=n^{-1} \beta^{-1 / 2}\left(\frac{2 a_{0} a_{0}^{\prime}}{a_{0}+a_{0}^{\prime}}\right)^{1 / 2} \int_{\mathcal{S}} V(x ; f) d x+o\left(n^{-1} \beta^{-1 / 2}\right) .
$$

Proof (i) Assumptions A1, A4'[1], and A5'(i) yield $0<\int_{\mathcal{S}} K(s ; x, \beta) f(s) d s \leq\|f\|_{\mathcal{S}}$ and

$$
\begin{aligned}
& \left|\int_{\mathcal{S}} K\left(s ; x, \beta / a_{0}\right) K\left(s ; x, \beta / a_{0}^{\prime}\right)(s-x) \int_{0}^{1} f^{\prime}(x+\theta(s-x)) d \theta d s\right| \\
& \leq\left\|f^{\prime}\right\|_{\mathcal{S}} \int_{\mathcal{S}}|s-x| K\left(s ; x, \beta / a_{0}\right) K\left(s ; x, \beta / a_{0}^{\prime}\right) d s=O(1) \quad \text { for } x \in \mathcal{S}_{I, \beta} \cup \mathcal{S}_{B, \beta, \kappa},
\end{aligned}
$$

since

$$
\int_{\mathcal{S}}|s-x| K\left(s ; x, \beta / a_{0}\right) K\left(s ; x, \beta / a_{0}^{\prime}\right) d s \leq \begin{cases}C_{K}^{\prime} a_{0}^{1 / 2}\{\beta \psi(x)\}^{-1 / 2} \mu_{2}^{1 / 2}\left(K\left(\cdot ; x, \beta / a_{0}^{\prime}\right)\right), & x \in \mathcal{S}_{I, \beta}, \\ C_{K} a_{0} \beta^{-1} \mu_{2}^{1 / 2}\left(K\left(\cdot ; x, \beta / a_{0}^{\prime}\right)\right), & x \in \mathcal{S}_{B, \beta, \kappa}\end{cases}
$$

Hence, under Assumption A2, we have

$$
\begin{aligned}
& \operatorname{Cov}\left[\widehat{f}_{\beta / a_{0}}(x), \widehat{f}_{\beta / a_{0}^{\prime}}(x)\right] \\
& =n^{-1}\left\{\int_{\mathcal{S}} K\left(s ; x, \beta / a_{0}\right) K\left(s ; x, \beta / a_{0}^{\prime}\right) f(s) d s-\int_{\mathcal{S}} K\left(s ; x, \beta / a_{0}\right) f(s) d s \int_{\mathcal{S}} K\left(s ; x, \beta / a_{0}^{\prime}\right) f(s) d s\right\} \\
& =n^{-1} \int_{\mathcal{S}} K\left(s ; x, \beta / a_{0}\right) K\left(s ; x, \beta / a_{0}^{\prime}\right)\left\{f(x)+(s-x) \int_{0}^{1} f^{\prime}(x+\theta(s-x)) d \theta\right\} d s+O\left(n^{-1}\right) \\
& = \begin{cases}n^{-1} \beta^{-1 / 2}\left(\frac{2 a_{0} a_{0}^{\prime}}{a_{0}+a_{0}^{\prime}}\right)^{1 / 2} V(x ; f)\left[1+O\left(\beta \psi^{-1}(x)\right)\right]+O\left(n^{-1}\right), x \in \mathcal{S}_{I, \beta}, \\
n^{-1} \beta^{-1} f(x)\left[\varsigma_{a_{0}, a_{0}^{\prime}}(\kappa)+\chi_{\left\{x \notin \mathcal{S}_{B}\right\}} o(1)\right]+O\left(n^{-1}\right), & x \in \mathcal{S}_{B, \beta, \kappa} .\end{cases}
\end{aligned}
$$

(ii) Note that, under Assumption A1(i), we have, for any interval $I(\subset \mathcal{S})$,

$$
\begin{aligned}
& \left|\int_{I} \operatorname{Cov}\left[\widehat{f}_{\beta / a_{0}}(x), \widehat{f}_{\beta / a_{0}^{\prime}}(x)\right] d x\right| \\
& \leq\left\{\int_{I} V\left[\widehat{f}_{\beta / a_{0}}(x)\right] d x \int_{I} V\left[\widehat{f}_{\beta / a_{0}^{\prime}}(x)\right] d x\right\}^{1 / 2} \\
& \leq n^{-1}\left\{\int_{I} \int_{\mathcal{S}} K^{2}\left(s ; x, \beta / a_{0}\right) f(s) d s d x \int_{I} \int_{\mathcal{S}} K^{2}\left(s ; x, \beta / a_{0}^{\prime}\right) f(s) d s d x\right\}^{1 / 2} \\
& \leq n^{-1} \beta^{-1} C_{K}\left\{a_{0} a_{0}^{\prime} \int_{I} \int_{\mathcal{S}} K\left(s ; x, \beta / a_{0}\right) f(s) d s d x \int_{I} \int_{\mathcal{S}} K\left(s ; x, \beta / a_{0}^{\prime}\right) f(s) d s d x\right\}^{1 / 2} .
\end{aligned}
$$

The case $\mathcal{S}=[0, \infty)$ : Under Assumption A1(i) and the boundedness of $f$, we can see that, choosing $\tau \in(1 / 2,1)$,

$$
\left|\int_{0}^{\beta^{\tau}} \operatorname{Cov}\left[\widehat{f}_{\beta / a_{0}}(x), \widehat{f}_{\beta / a_{0}^{\prime}}(x)\right] d x\right| \leq n^{-1} \beta^{\tau-1} C_{K}\left(a_{0} a_{0}^{\prime}\right)^{1 / 2}\|f\|_{[0, \infty)}=o\left(n^{-1} \beta^{-1 / 2}\right) .
$$


Under Assumptions A1(i), A3, and A5'(ii), the choice of $\tau^{\prime} \in\left(1 /\left\{2\left(k^{\prime}+1\right)\right\}, 1 / 2\right)$ yields

$$
\begin{aligned}
& \left|\int_{\beta^{-\tau^{\prime}}}^{\infty} \operatorname{Cov}\left[\widehat{f}_{\beta / a_{0}}(x), \widehat{f}_{\beta / a_{0}^{\prime}}(x)\right] d x\right| \\
& \leq n^{-1} \beta^{-1} C_{K}\left\{a_{0} a_{0}^{\prime} \int_{0}^{\infty} \int_{\beta^{-\tau^{\prime}}}^{\infty} K\left(s ; x, \beta / a_{0}\right) d x f(s) d s \int_{0}^{\infty} \int_{\beta^{-\tau^{\prime}}}^{\infty} K\left(s ; x, \beta / a_{0}^{\prime}\right) d x f(s) d s\right\}^{1 / 2} \\
& =O\left(n^{-1} \beta^{\tau^{\prime}\left(k^{\prime}+1\right)-1}\right)=o\left(n^{-1} \beta^{-1 / 2}\right) .
\end{aligned}
$$

Also,

$$
\begin{aligned}
n^{-1} \int_{\beta^{\tau}}^{\beta^{-\tau^{\prime}}}\left\{\beta^{1 / 2} \frac{f(x)}{\sqrt{\psi^{3}(x)}}+1\right\} d x & \leq n^{-1}\left\{\beta^{1 / 2-\tau} \int_{0}^{\infty} \frac{f(x)}{\sqrt{\psi(x)}} d x+\beta^{-\tau^{\prime}}\right\}=o\left(n^{-1} \beta^{-1 / 2}\right), \\
n^{-1} \beta^{-1 / 2}\left(\int_{0}^{\beta^{\tau}}+\int_{\beta^{-\tau^{\prime}}}^{\infty}\right) \frac{f(x)}{\sqrt{\psi(x)}} d x & \leq n^{-1} \beta^{-1 / 2}\left\{\|f\|_{[0, \infty)} \int_{0}^{\beta^{\tau}} \frac{1}{\sqrt{\psi(x)}} d x+\beta^{\tau^{\prime} / 2} \int_{\beta^{-\tau^{\prime}}}^{\infty} f(x) d x\right\} \\
& =o\left(n^{-1} \beta^{-1 / 2}\right) .
\end{aligned}
$$

Combining them with the result (i) yields

$$
\begin{aligned}
& \left|\int_{0}^{\infty} \operatorname{Cov}\left[\widehat{f}_{\beta / a_{0}}(x), \widehat{f}_{\beta / a_{0}^{\prime}}(x)\right] d x-n^{-1} \beta^{-1 / 2}\left(\frac{2 a_{0} a_{0}^{\prime}}{a_{0}+a_{0}^{\prime}}\right)^{1 / 2} \int_{0}^{\infty} V(x ; f) d x\right| \\
& \leq \int_{\beta^{\tau}}^{\beta^{-\tau^{\prime}}}\left|\operatorname{Cov}\left[\widehat{f}_{\beta / a_{0}}(x), \widehat{f}_{\beta / a_{0}^{\prime}}(x)\right]-n^{-1} \beta^{-1 / 2}\left(\frac{2 a_{0} a_{0}^{\prime}}{a_{0}+a_{0}^{\prime}}\right)^{1 / 2} V(x ; f)\right| d x+o\left(n^{-1} \beta^{-1 / 2}\right) \\
& =o\left(n^{-1} \beta^{-1 / 2}\right) .
\end{aligned}
$$

The case $\mathcal{S}=[0,1]$ : Under Assumption A1(i) and the boundedness of $f$, we can see that, choosing $\tau \in(1 / 2,1)$,

$$
\left|\left(\int_{0}^{\beta^{\tau}}+\int_{1-\beta^{\tau}}^{1}\right) \operatorname{Cov}\left[\widehat{f}_{\beta / a_{0}}(x), \widehat{f}_{\beta / a_{0}^{\prime}}(x)\right] d x\right| \leq 2 n^{-1} \beta^{\tau-1} C_{K}\left(a_{0} a_{0}^{\prime}\right)^{1 / 2}\|f\|_{[0,1]}=o\left(n^{-1} \beta^{-1 / 2}\right) .
$$

Also,

$$
\begin{aligned}
& n^{-1} \int_{\beta^{\tau}}^{1-\beta^{\tau}}\left\{\frac{\beta^{1 / 2}}{\sqrt{\psi^{3}(x)}}+1\right\} d x \leq n^{-1}\left\{\frac{\beta^{1 / 2}}{\beta^{\tau}\left(1-\beta^{\tau}\right)} \int_{0}^{1} \frac{1}{\sqrt{\psi(x)}} d x+1\right\}=o\left(n^{-1} \beta^{-1 / 2}\right), \\
& n^{-1} \beta^{-1 / 2}\left(\int_{0}^{\beta^{\tau}}+\int_{1-\beta^{\tau}}^{1}\right) \frac{1}{\sqrt{\psi(x)}} d x=o\left(n^{-1} \beta^{-1 / 2}\right) .
\end{aligned}
$$

Combining them with the result (i) yields

$$
\begin{aligned}
& \left|\int_{0}^{1} \operatorname{Cov}\left[\widehat{f}_{\beta / a_{0}}(x), \widehat{f}_{\beta / a_{0}^{\prime}}(x)\right] d x-n^{-1} \beta^{-1 / 2}\left(\frac{2 a_{0} a_{0}^{\prime}}{a_{0}+a_{0}^{\prime}}\right)^{1 / 2} \int_{0}^{1} V(x ; f) d x\right| \\
& \leq \int_{\beta^{\tau}}^{1-\beta^{\tau}}\left|\operatorname{Cov}\left[\widehat{f}_{\beta / a_{0}}(x), \widehat{f}_{\beta / a_{0}^{\prime}}(x)\right]-n^{-1} \beta^{-1 / 2}\left(\frac{2 a_{0} a_{0}^{\prime}}{a_{0}+a_{0}^{\prime}}\right)^{1 / 2} V(x ; f)\right| d x+o\left(n^{-1} \beta^{-1 / 2}\right) \\
& =o\left(n^{-1} \beta^{-1 / 2}\right) .
\end{aligned}
$$




\section{Appendix A.2. Original estimator (without bias corrections)}

In this section, we prove Theorems 1-3.

Proof of Theorem 1 (i) Under Assumption A5[p](i,ii), the $2 p$-term Taylor expansion of $f$ around $s=x$ yields

$$
\begin{aligned}
E\left[\widehat{f}_{\beta}(x)\right] & =\int_{\mathcal{S}} K(s ; x, \beta) f(s) d s \\
& =\int_{\mathcal{S}} K(s ; x, \beta)\left\{f(x)+\sum_{j=1}^{2 p} \frac{1}{j !}(s-x)^{j} f^{(j)}(x)\right\} d s+\mathcal{R}_{\beta}(x) \\
& =f(x)+\sum_{j=1}^{2 p} \frac{1}{j !} \mu_{j}(K(\cdot ; x, \beta)) f^{(j)}(x)+\mathcal{R}_{\beta}(x),
\end{aligned}
$$

where

$$
\mathcal{R}_{\beta}(x)=\frac{1}{(2 p-1) !} \int_{\mathcal{S}} K(s ; x, \beta)(s-x)^{2 p} \int_{0}^{1}\left\{f^{(2 p)}(x+\theta(s-x))-f^{(2 p)}(x)\right\}(1-\theta)^{2 p-1} d \theta d s
$$

satisfies

$$
\left|\mathcal{R}_{\beta}(x)\right| \leq \frac{L_{2 p}}{(2 p) !} \int_{\mathcal{S}}|s-x|^{2 p+\eta_{2 p}} K(s ; x, \beta) d s \leq \frac{L_{2 p}}{(2 p) !} \mu_{2(p+1)}^{\left(2 p+\eta_{2 p}\right) /\{2(p+1)\}}(K(\cdot ; x, \beta)) .
$$

The result follows from Assumption $44[p]$, i.e., when $\mathcal{S}=[0, \infty)$,

$$
\mu_{j}(K(\cdot ; x, \beta))= \begin{cases}\sum_{k=\lceil j / 2\rceil}^{p} \chi_{\{k \leq j\}} \zeta_{j, k} x^{j-k} \beta^{k}+O\left(\beta^{p+1}(1+x)^{p-1}\right), & j=1, \ldots, 2 p, \\ O\left(\beta^{p+1}(1+x)^{p+1}\right), & j=2(p+1),\end{cases}
$$

hence,

$$
\begin{aligned}
& \sum_{j=1}^{2 p} \mu_{j}(K(\cdot ; x, \beta)) \frac{f^{(j)}(x)}{j !}+\mathcal{R}_{\beta}(x) \\
&=\sum_{m=1}^{p} \sum_{k=m}^{p}\left\{\chi_{\{k \leq 2 m-1\}} \zeta_{2 m-1, k} x^{2 m-1-k} \beta^{k} \frac{f^{(2 m-1)}(x)}{(2 m-1) !}+\chi_{\{k \leq 2 m\}} \zeta_{2 m, k} x^{2 m-k} \beta^{k} \frac{f^{(2 m)}(x)}{(2 m) !}\right\} \\
&+O\left(\beta^{p+1}(1+x)^{p-1}\right)+O\left(\beta^{p+\eta_{2 p} / 2}(1+x)^{p+\eta_{2 p} / 2}\right) \\
&= \sum_{k=1}^{p} \beta^{k} \sum_{m=1}^{k}\left\{\chi_{\{k \leq 2 m-1\}} \zeta_{2 m-1, k} x^{2 m-1-k} \frac{f^{(2 m-1)}(x)}{(2 m-1) !}+\chi_{\{k \leq 2 m\}} \zeta_{2 m, k} x^{2 m-k} \frac{f^{(2 m)}(x)}{(2 m) !}\right\} \\
&+O\left(\beta^{p+\eta_{2 p} / 2}(1+x)^{p+\eta_{2 p} / 2}\right) \\
&= \sum_{k=1}^{p} \beta^{k} \gamma_{k}(x ; f)+O\left(\beta^{p+\eta_{2 p} / 2}(1+x)^{p+\eta_{2 p} / 2}\right),
\end{aligned}
$$


with $\gamma_{k}(x ; f)=\sum_{j=1}^{2 k} \chi_{\{k \leq j\}} \zeta_{j, k} x^{j-k} f^{(j)}(x) / j$ !, whereas, when $\mathcal{S}=[0,1]$, uniformly in $x \in[0,1]$,

$$
\begin{aligned}
& \sum_{j=1}^{2 p} \mu_{j}(K(\cdot ; x, \beta)) \frac{f^{(j)}(x)}{j !}+\mathcal{R}_{\beta}(x) \\
& =\sum_{m=1}^{p}\left\{\sum_{k=m}^{p} \zeta_{2 m-1, k}(x) \beta^{k} \frac{f^{(2 m-1)}(x)}{(2 m-1) !}+\sum_{k=m}^{p} \zeta_{2 m, k}(x) \beta^{k} \frac{f^{(2 m)}(x)}{(2 m) !}\right\}+O\left(\beta^{p+1}\right)+O\left(\beta^{p+\eta_{2 p} / 2}\right) \\
& =\sum_{k=1}^{p} \beta^{k}\left\{\sum_{m=1}^{k} \zeta_{2 m-1, k}(x) \frac{f^{(2 m-1)}(x)}{(2 m-1) !}+\sum_{m=1}^{k} \zeta_{2 m, k}(x) \frac{f^{(2 m)}(x)}{(2 m) !}\right\}+O\left(\beta^{p+\eta_{2 p} / 2}\right) \\
& =\sum_{k=1}^{p} \beta^{k} \gamma_{k}(x ; f)+O\left(\beta^{p+\eta_{2 p} / 2}\right),
\end{aligned}
$$

with $\gamma_{k}(x ; f)=\sum_{j=1}^{2 k} \zeta_{j, k}(x) f^{(j)}(x) / j$ !

(ii) Use Lemma A.2(i) (set $a_{0}=a_{0}^{\prime}=1$ ).

(iii) Use Lemma A.1(ii) and the Borel-Cantelli lemma.

Remark A.1 Assumption A4[p] for some $p \in \mathbb{N} \backslash\{1\}$ implies that ${ }^{[9]}$ Assumption $\mathrm{A} 4^{\prime}[J]$ holds for $J=2, \ldots, p$. Then, under Assumption A5[J](i) (which is, of course, implied by A5[p](i)), we have

$$
\begin{aligned}
& \text { when } \mathcal{S}=[0, \infty), \operatorname{Bias}\left[\widehat{f}_{\beta}(x)\right]=\sum_{k=1}^{J-1} \beta^{k} \gamma_{k}(x ; f)+O\left(\beta^{J}(1+x)^{J}\right), \\
& \text { when } \mathcal{S}=[0,1], \text { uniformly in } x \in[0,1], \operatorname{Bias}\left[\widehat{f}_{\beta}(x)\right]=\sum_{k=1}^{J-1} \beta^{k} \gamma_{k}(x ; f)+O\left(\beta^{J}\right) .
\end{aligned}
$$

The proofs of (A.1) and (A.1 $\left.1^{\prime}\right)$ for the case $J=2, \ldots, p$ (also $(2)$ and $\left(2^{\prime}\right)$ for the case $J=1$ ) are easy, as follows: we have, as in Proof of Theorem 1(i),

$$
E\left[\widehat{f}_{\beta}(x)\right]=f(x)+\sum_{j=1}^{2 J-1} \frac{1}{j !} \mu_{j}(K(\cdot ; x, \beta)) f^{(j)}(x)+\mathcal{R}_{\beta}^{\dagger}(x),
$$

${ }^{[9]}$ The case $\mathcal{S}=[0,1]$ is trivial. When $\mathcal{S}=[0, \infty)$, it holds that, for $j=1, \ldots, 2 J-2($ note $J=2, \ldots, p)$

$$
\begin{aligned}
\mu_{j}(K(\cdot ; x, \beta)) & =\sum_{k=\lceil j / 2\rceil}^{J-1} \chi_{\{k \leq j\}} \zeta_{j, k} x^{j-k} \beta^{k}+\sum_{k=J}^{p} \chi_{\{k \leq j\}} \zeta_{j, k} x^{j-k} \beta^{k}+\chi_{\{j>p\}} O\left(\beta^{J}(1+x)^{j-(p+1)}\right) \\
& =\sum_{k=\lceil j / 2\rceil}^{J-1} \chi_{\{k \leq j\}} \zeta_{j, k} x^{j-k} \beta^{k}+\chi_{\{j>J-1\}} O\left(\beta^{J}(1+x)^{j-J}\right),
\end{aligned}
$$

and that

$$
\begin{aligned}
\mu_{2 J-1}(K(\cdot ; x, \beta)) & =\sum_{k=J}^{p} \chi_{\{k \leq 2 J-1\}} \zeta_{2 J-1, k} x^{2 J-1-k} \beta^{k}+\chi_{\{2 J-1>p\}} O\left(\beta^{J}(1+x)^{J-2}\right)=O\left(\beta^{J}(1+x)^{J-1}\right), \\
\mu_{2 J}(K(\cdot ; x, \beta)) & =\sum_{k=J}^{p} \chi_{\{k \leq 2 J\}} \zeta_{2 J-1, k} x^{2 J-k} \beta^{k}+\chi_{\{2 J>p\}} O\left(\beta^{J}(1+x)^{J-1}\right)=O\left(\beta^{J}(1+x)^{J}\right) .
\end{aligned}
$$


where

$$
\mathcal{R}_{\beta}^{\dagger}(x)=\frac{1}{(2 J-1) !} \int_{\mathcal{S}} K(s ; x, \beta)(s-x)^{2 J} \int_{0}^{1} f^{(2 J)}(x+\theta(s-x))(1-\theta)^{2 J-1} d \theta d s
$$

satisfies

$$
\left|\mathcal{R}_{\beta}^{\dagger}(x)\right| \leq \frac{\left\|f^{(2 J)}\right\|_{\mathcal{S}}}{(2 J) !} \int_{\mathcal{S}}(s-x)^{2 J} K(s ; x, \beta) d s=\frac{\left\|f^{(2 J)}\right\|_{\mathcal{S}}}{(2 J) !} \mu_{2 J}(K(\cdot ; x, \beta)) .
$$

It follows that, when $\mathcal{S}=[0, \infty)$,

$$
\begin{aligned}
& \sum_{j=1}^{2 J-1} \mu_{j}(K(\cdot ; x, \beta)) \frac{f^{(j)}(x)}{j !}+\mathcal{R}_{\beta}^{\dagger}(x) \\
= & \sum_{m=1}^{J-1}\left\{\sum_{k=m}^{J-1} \chi_{\{k \leq 2 m-1\}} \zeta_{2 m-1, k} x^{2 m-1-k} \beta^{k} \frac{f^{(2 m-1)}(x)}{(2 m-1) !}+\sum_{k=m}^{J-1} \chi_{\{k \leq 2 m\}} \zeta_{2 m, k} x^{2 m-k} \beta^{k} \frac{f^{(2 m)}(x)}{(2 m) !}\right\} \\
& +O\left(\beta^{J}(1+x)^{J-1}\right)+O\left(\beta^{J}(1+x)^{J}\right) \\
= & \sum_{k=1}^{J-1} \beta^{k}\left\{\sum_{m=1}^{k} \chi_{\{k \leq 2 m-1\}} \zeta_{2 m-1, k} x^{2 m-1-k} \frac{f^{(2 m-1)}(x)}{(2 m-1) !}+\sum_{m=1}^{k} \chi_{\{k \leq 2 m\}} \zeta_{2 m, k} x^{2 m-k} \frac{f^{(2 m)}(x)}{(2 m) !}\right\} \\
& +O\left(\beta^{J}(1+x)^{J}\right) \\
= & \sum_{k=1}^{J-1} \beta^{k} \gamma_{k}(x ; f)+O\left(\beta^{J}(1+x)^{J}\right),
\end{aligned}
$$

whereas, when $\mathcal{S}=[0,1]$, uniformly in $x \in[0,1]$,

$$
\begin{aligned}
& \sum_{j=1}^{2 J-1} \mu_{j}(K(\cdot ; x, \beta)) \frac{f^{(j)}(x)}{j !}+\mathcal{R}_{\beta}^{\dagger}(x) \\
= & \sum_{m=1}^{J-1}\left\{\sum_{k=m}^{J-1} \zeta_{2 m-1, k}(x) \beta^{k} \frac{f^{(2 m-1)}(x)}{(2 m-1) !}+\sum_{k=m}^{J-1} \zeta_{2 m, k}(x) \beta^{k} \frac{f^{(2 m)}(x)}{(2 m) !}\right\}+O\left(\beta^{J}\right) \\
= & \sum_{k=1}^{J-1} \beta^{k}\left\{\sum_{m=1}^{k} \zeta_{2 m-1, k}(x) \frac{f^{(2 m-1)}(x)}{(2 m-1) !}+\sum_{m=1}^{k} \zeta_{2 m, k}(x) \frac{f^{(2 m)}(x)}{(2 m) !}\right\}+O\left(\beta^{J}\right) \\
= & \sum_{k=1}^{J-1} \beta^{k} \gamma_{k}(x ; f)+O\left(\beta^{J}\right) .
\end{aligned}
$$

Proof of Theorem 2 Under Assumption A1, we have

$$
\sup _{s \in \mathcal{S}}|\Delta(s ; x, \beta)| \leq\left\{\begin{array}{l}
C_{K}^{\prime}\{\beta \psi(x)\}^{-1 / 2} \quad \text { for fixed } x \in \mathcal{S}_{I}, \\
C_{K} \beta^{-1} \text { for } x \in \mathcal{S}_{B} .
\end{array}\right.
$$

Also, from Theorem 1(ii),

$$
\begin{aligned}
\lim _{n \rightarrow \infty} n \beta^{1 / 2} V\left[\widehat{f}_{\beta}(x)\right] & =V(x ; f) \quad \text { for fixed } x \in \mathcal{S}_{I}, \\
\lim _{n \rightarrow \infty} n \beta V\left[\widehat{f}_{\beta}(x)\right] & =\varsigma_{1,1}(0) f(x) \quad \text { for } x \in \mathcal{S}_{B} .
\end{aligned}
$$

Noting that $V\left[\widehat{f}_{\beta}(x)\right]=V\left[\sum_{i=1}^{n} n^{-1} \Delta\left(X_{i} ; x, \beta\right)\right]=\sum_{i=1}^{n} n^{-2} E\left[\Delta^{2}\left(X_{i} ; x, \beta\right)\right]$, we have

$$
\frac{\sum_{i=1}^{n} E\left[\left|n^{-1} \Delta\left(X_{i} ; x, \beta\right)\right|^{2+\delta}\right]}{\left\{\sum_{i=1}^{n} V\left[n^{-1} \Delta\left(X_{i} ; x, \beta\right)\right]\right\}^{1+\delta / 2}}=\left\{\begin{array}{l}
O\left(\left(n \beta^{1 / 2}\right)^{-\delta / 2}\right) \text { for fixed } x \in \mathcal{S}_{I}, \\
O\left((n \beta)^{-\delta / 2}\right) \text { for } x \in \mathcal{S}_{B},
\end{array}\right.
$$


where $\delta>0$ is arbitrary. Hence, Lyapunov's central limit theorem enables us to see that

$$
\frac{\widehat{f}_{\beta}(x)-E\left[\widehat{f}_{\beta}(x)\right]}{\left\{V\left[\widehat{f}_{\beta}(x)\right]\right\}^{1 / 2}} \stackrel{d}{\rightarrow} N(0,1) \quad \text { for fixed } x \in \mathcal{S} .
$$

The results follow from Slutsky's lemma.

Proof of Theorem 3 The integrated variance approximation easily follows from Lemma A.2(ii) (set $a_{0}=a_{0}^{\prime}=1$ ). It suffices to approximate the integrated squared bias.

The case $\mathcal{S}=[0,1]$ : Theorem $1(\mathrm{i})$ immediately yields

$$
\int_{0}^{1}\left\{\operatorname{Bias}\left[\widehat{f}_{\beta}(x)\right]\right\}^{2} d x=\beta^{2} \int_{0}^{1} \gamma_{1}^{2}(x ; f) d x+o\left(\beta^{2}\right) .
$$

The case $\mathcal{S}=[0, \infty)$ : Under Assumptions A3 and A5[1](iii) and the boundedness of $f$, we have

$$
\begin{aligned}
\int_{\beta^{-\tau_{2}}}^{\infty}\left\{\operatorname{Bias}\left[\widehat{f}_{\beta}(x)\right]\right\}^{2} d x & \leq 2 \int_{\beta^{-\tau_{2}}}^{\infty}\left[\left\{\int_{0}^{\infty} K(s ; x, \beta) f(s) d s\right\}^{2}+f^{2}(x)\right] d x \\
& \leq 2\|f\|_{[0, \infty)}\left\{\int_{0}^{\infty} \int_{\beta^{-\tau_{2}}}^{\infty} K(s ; x, \beta) d x f(s) d s+\int_{\beta^{-\tau_{2}}}^{\infty} f(x) d x\right\} \\
& =O\left(\beta^{\tau_{2}\left(k_{2}+1\right)}\right)=o\left(\beta^{2}\right) .
\end{aligned}
$$

Theorem 1(i) and Assumption A5[1](iii) (note that $\int_{0}^{\beta^{-\tau_{2}}} \mathcal{E}_{\beta, 1}^{2}(x) d x=o\left(\beta^{2}\right)$ ) yield

$$
\begin{aligned}
& \left|\int_{0}^{\beta^{-\tau_{2}}}\left\{\operatorname{Bias}\left[\widehat{f}_{\beta}(x)\right]\right\}^{2} d x-\beta^{2} \int_{0}^{\beta^{-\tau_{2}}} \gamma_{1}^{2}(x ; f) d x\right| \\
& \leq 2 \beta\left\{\int_{0}^{\infty} \gamma_{1}^{2}(x ; f) d x \int_{0}^{\beta^{-\tau_{2}}} \mathcal{E}_{\beta, 1}^{2}(x) d x\right\}^{1 / 2}+\int_{0}^{\beta^{-\tau_{2}}} \mathcal{E}_{\beta, 1}^{2}(x) d x=o\left(\beta^{2}\right) .
\end{aligned}
$$

\section{Appendix A.3. Proof of Lemma 4}

Proof of Lemma 4 Let $Z=\operatorname{diag}\left(z_{1}, \ldots, z_{p}\right)$. Then, $\prod_{j=1}^{p} z_{j}=|Z|=|\mathcal{V}(\boldsymbol{z})||Z|\left|\mathcal{V}^{-1}(\boldsymbol{z})\right|=$ $\left|\mathcal{V}(\boldsymbol{z}) Z \mathcal{V}^{-1}(\boldsymbol{z})\right|$, where $|\cdot|$ denotes the determinant. Also, it is not difficult to see that

$$
\begin{aligned}
& \mathcal{V}(\boldsymbol{z}) Z \mathcal{V}^{-1}(\boldsymbol{z})=\left(\begin{array}{cccc}
z_{1} & z_{2} & \cdots & z_{p} \\
z_{1}^{2} & z_{2}^{2} & \cdots & z_{p}^{2} \\
\vdots & \vdots & \ddots & \vdots \\
z_{1}^{p} & z_{2}^{p} & \cdots & z_{p}^{p}
\end{array}\right)\left(\begin{array}{cccc}
{\left[\mathcal{V}^{-1}(\boldsymbol{z})\right]_{11}} & {\left[\mathcal{V}^{-1}(\boldsymbol{z})\right]_{12}} & \cdots & {\left[\mathcal{V}^{-1}(\boldsymbol{z})\right]_{1 p}} \\
{\left[\mathcal{V}^{-1}(\boldsymbol{z})\right]_{21}} & {\left[\mathcal{V}^{-1}(\boldsymbol{z})\right]_{22}} & \cdots & {\left[\mathcal{V}^{-1}(\boldsymbol{z})\right]_{2 p}} \\
\vdots & \vdots & \ddots & \vdots \\
{\left[\mathcal{V}^{-1}(\boldsymbol{z})\right]_{p 1}} & {\left[\mathcal{V}^{-1}(\boldsymbol{z})\right]_{p 2}} & \cdots & {\left[\mathcal{V}^{-1}(\boldsymbol{z})\right]_{p p}}
\end{array}\right) \\
& =\left(\begin{array}{cccc}
\sum_{j=1}^{p} z_{j}\left[\mathcal{V}^{-1}(\boldsymbol{z})\right]_{j 1} & \sum_{j=1}^{p} z_{j}\left[\mathcal{V}^{-1}(\boldsymbol{z})\right]_{j 2} & \cdots & \sum_{j=1}^{p} z_{j}\left[\mathcal{V}^{-1}(\boldsymbol{z})\right]_{j p} \\
\sum_{j=1}^{p} z_{j}^{2}\left[\mathcal{V}^{-1}(\boldsymbol{z})\right]_{j 1} & \sum_{j=1}^{p} z_{j}^{2}\left[\mathcal{V}^{-1}(\boldsymbol{z})\right]_{j 2} & \cdots & \sum_{j=1}^{p} z_{j}^{2}\left[\mathcal{V}^{-1}(\boldsymbol{z})\right]_{j p} \\
\vdots & \vdots & \ddots & \vdots \\
\sum_{j=1}^{p} z_{j}^{p}\left[\mathcal{V}^{-1}(\boldsymbol{z})\right]_{j 1} & \sum_{j=1}^{p} z_{j}^{p}\left[\mathcal{V}^{-1}(\boldsymbol{z})\right]_{j 2} & \cdots & \sum_{j=1}^{p} z_{j}^{p}\left[\mathcal{V}^{-1}(\boldsymbol{z})\right]_{j p}
\end{array}\right)
\end{aligned}
$$

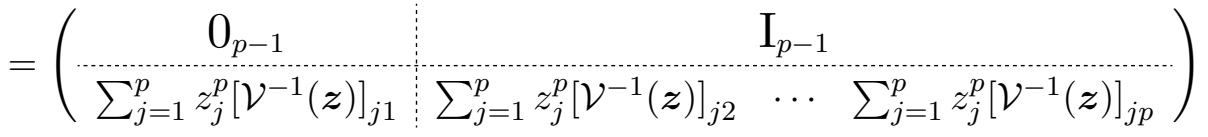


(the last equality is a direct consequence of $\mathcal{V}(\boldsymbol{z}) \mathcal{V}^{-1}(\boldsymbol{z})=I_{p}$, i.e., $\sum_{j=1}^{p} z_{j}^{k-1}\left[\mathcal{V}^{-1}(\boldsymbol{z})\right]_{j k}=1$ and $\sum_{j=1}^{p} z_{j}^{\ell-1}\left[\mathcal{V}^{-1}(\boldsymbol{z})\right]_{j k}=0$ for $\left.k=1, \ldots, p ; \ell \in\{1, \ldots, p\} \backslash\{k\}\right)$, hence,

$$
\left|\mathcal{V}(\boldsymbol{z}) Z \mathcal{V}^{-1}(\boldsymbol{z})\right|=(-1)^{p-1} \sum_{j=1}^{p} z_{j}^{p}\left[\mathcal{V}^{-1}(\boldsymbol{z})\right]_{j 1}
$$

\section{Appendix A.4. Additive estimator}

In this section, we prove Theorems 5-7, with a slight modification of Proofs of Theorems 1-3.

Proof of Theorem 5 Theorem 1(i), together with (7) and (8), yields the result (i). Using

$$
V\left[\widehat{f}_{\beta, A D D_{\boldsymbol{a}}^{p}}(x)\right]=\sum_{j=1}^{p} \sum_{j^{\prime}=1}^{p} c_{j}(\boldsymbol{a}) c_{j^{\prime}}(\boldsymbol{a}) \operatorname{Cov}\left[\widehat{f}_{\beta / a_{j}}(x), \widehat{f}_{\beta / a_{j^{\prime}}}(x)\right],
$$

Lemma A.2(i) yields the result (ii).

Proof of Theorem 6 Under Assumption A1, we have

$$
\sup _{s \in \mathcal{S}}\left|\Delta_{A D D_{a}^{p}}(s ; x, \beta)\right| \leq\left\{\begin{array}{l}
\sum_{k=1}^{p}\left|c_{k}(\boldsymbol{a})\right| C_{K}^{\prime} a_{k}^{1 / 2}\{\beta \psi(x)\}^{-1 / 2} \quad \text { for fixed } x \in \mathcal{S}_{I}, \\
\sum_{k=1}^{p}\left|c_{k}(\boldsymbol{a})\right| C_{K} a_{k} \beta^{-1} \quad \text { for } x \in \mathcal{S}_{B},
\end{array}\right.
$$

since $\Delta_{A D D_{\boldsymbol{a}}^{p}}(s ; x, \beta)=\sum_{k=1}^{p} c_{k}(\boldsymbol{a}) \Delta\left(s ; x, \beta / a_{k}\right)$ (we used (A.2)). Also, from Theorem 5(ii),

$$
\begin{aligned}
& \lim _{n \rightarrow \infty} n \beta^{1 / 2} V\left[\widehat{f}_{\beta, A D D_{a}^{p}}(x)\right]=\lambda_{p, \boldsymbol{a}} V(x ; f) \quad \text { for fixed } x \in \mathcal{S}_{I}, \\
& \lim _{n \rightarrow \infty} n \beta V\left[\widehat{f}_{\beta, A D D_{\boldsymbol{a}}^{p}}(x)\right]=v_{p, \boldsymbol{a}}(0) f(x) \quad \text { for } x \in \mathcal{S}_{B} .
\end{aligned}
$$

Noting that $V\left[\widehat{f}_{\beta, A D D_{\boldsymbol{a}}^{p}}(x)\right]=V\left[\sum_{i=1}^{n} n^{-1} \Delta_{A D D_{\boldsymbol{a}}^{p}}\left(X_{i} ; x, \beta\right)\right]=\sum_{i=1}^{n} n^{-2} E\left[\Delta_{A D D_{\boldsymbol{a}}^{p}}^{2}\left(X_{i} ; x, \beta\right)\right]$, we have

$$
\frac{\sum_{i=1}^{n} E\left[\left|n^{-1} \Delta_{A D D_{a}^{p}}\left(X_{i} ; x, \beta\right)\right|^{2+\delta}\right]}{\left\{\sum_{i=1}^{n} V\left[n^{-1} \Delta_{A D D_{a}^{p}}\left(X_{i} ; x, \beta\right)\right]\right\}^{1+\delta / 2}}=\left\{\begin{array}{l}
O\left(\left(n \beta^{1 / 2}\right)^{-\delta / 2}\right) \text { for fixed } x \in \mathcal{S}_{I}, \\
O\left((n \beta)^{-\delta / 2}\right) \text { for } x \in \mathcal{S}_{B},
\end{array}\right.
$$

where $\delta>0$ is arbitrary. Hence, Lyapunov's central limit theorem enables us to see that

$$
\frac{\widehat{f}_{\beta, A D D_{\boldsymbol{a}}^{p}}(x)-E\left[\widehat{f}_{\beta, A D D_{\boldsymbol{a}}^{p}}(x)\right]}{\left\{V\left[\widehat{f}_{\beta, A D D_{\boldsymbol{a}}^{p}}(x)\right]\right\}^{1 / 2}} \stackrel{d}{\rightarrow} N(0,1) \quad \text { for fixed } x \in \mathcal{S} .
$$

The results follow from Slutsky's lemma.

Proof of Theorem 7 The integrated variance approximation easily follows from (A.3) and Lemma A.2(ii). It suffices to approximate the integrated squared bias.

The case $\mathcal{S}=[0,1]$ : Theorem $5(\mathrm{i})$ immediately yields

$$
\int_{0}^{1}\left\{\operatorname{Bias}\left[\widehat{f}_{\beta, A D D_{\boldsymbol{a}}^{p}}(x)\right]\right\}^{2} d x=\beta^{2 p} \int_{0}^{1} B_{p, \boldsymbol{a}}^{2}(x ; f) d x+o\left(\beta^{2 p}\right) .
$$


The case $\mathcal{S}=[0, \infty)$ : Under Assumptions A3 and A5[p](iii) and the boundedness of $f$, we have

$$
\begin{aligned}
& \int_{\beta^{-\tau_{2} p}}^{\infty}\left\{\operatorname{Bias}\left[\widehat{f}_{\beta, A D D_{\boldsymbol{a}}^{p}}(x)\right]\right\}^{2} d x \\
& \leq 2 \int_{\beta^{-\tau_{2 p}}}^{\infty}\left[\left\{\int_{0}^{\infty} K_{A D D_{\boldsymbol{a}}^{p}}(s ; x, \beta) f(s) d s\right\}^{2}+f^{2}(x)\right] d x \\
& \leq 2\|f\|_{[0, \infty)}\left[\left\{\sum_{k=1}^{p}\left|c_{k}(\boldsymbol{a})\right|\right\} \sum_{k=1}^{p}\left|c_{k}(\boldsymbol{a})\right| \int_{0}^{\infty} \int_{\beta^{-\tau_{2 p}}}^{\infty} K\left(s ; x, \beta / a_{k}\right) d x f(s) d s+\int_{\beta^{-\tau_{2 p}}}^{\infty} f(x) d x\right] \\
& =O\left(\beta^{\tau_{2 p}\left(k_{2 p}+1\right)}\right)=o\left(\beta^{2 p}\right) .
\end{aligned}
$$

Theorem 5(i) and Assumption A5[p](iii) (note that $\int_{0}^{\beta^{-\tau_{2 p}}} \mathcal{E}_{\beta, A D D_{\boldsymbol{a}}^{p}}^{2}(x) d x=o\left(\beta^{2 p}\right)$ ) yield

$$
\begin{aligned}
& \left|\int_{0}^{\beta^{-\tau_{2 p}}}\left\{\operatorname{Bias}\left[\widehat{f}_{\beta, A D D_{\boldsymbol{a}}^{p}}(x)\right]\right\}^{2} d x-\beta^{2 p} \int_{0}^{\beta^{-\tau_{2 p}}} B_{p, \boldsymbol{a}}^{2}(x ; f) d x\right| \\
& \leq 2 \beta^{p}\left\{\int_{0}^{\infty} B_{p, \boldsymbol{a}}^{2}(x ; f) d x \int_{0}^{\beta^{-\tau_{2 p}}} \mathcal{E}_{\beta, A D D_{\boldsymbol{a}}^{p}}^{2}(x) d x\right\}^{1 / 2}+\int_{0}^{\beta^{-\tau_{2 p}}} \mathcal{E}_{\beta, A D D_{\boldsymbol{a}}^{p}}^{2}(x) d x=o\left(\beta^{2 p}\right) .
\end{aligned}
$$

\section{Appendix A.5. TS-type and JF-type estimators}

In this section, we will prove Theorems $8-10$ and $8^{\prime}-10^{\prime}$. For this, we prepare the stochastic expansions of the TS-type and JF-type estimators (5) and (6), together with technical lemmas.

\section{A.5.1. Stochastic expansion of TS-type estimator and auxiliary lemmas}

Write $\bar{D}_{\beta / a_{k}}(x)=\widehat{f}_{\beta / a_{k}}(x)+\epsilon / a_{k}-f(x)$. Whenever $f(x)>0$, we have

$$
\begin{aligned}
\widehat{f}_{\beta, T S_{\boldsymbol{a}}^{p}}(x) & =f(x) \exp \left[\sum_{k=1}^{p} c_{k}(\boldsymbol{a}) \log \left\{1+\frac{\bar{D}_{\beta / a_{k}}(x)}{f(x)}\right\}\right] \\
& =f(x) \exp \left\{\mathcal{Q}_{\beta, T S_{\boldsymbol{a}}^{p}}(x)+\mathcal{R}_{\beta, p+1}(x)\right\} \\
& =f(x)+\sum_{i=1}^{p} \frac{f(x)}{i !} \mathcal{Q}_{\beta, T S_{\boldsymbol{a}}^{p}}^{i}(x)+\mathcal{R}_{\beta, T S_{\boldsymbol{a}}^{p}}(x),
\end{aligned}
$$

where

$$
\begin{aligned}
\mathcal{Q}_{\beta, T S_{\boldsymbol{a}}^{p}}(x)= & \sum_{j=1}^{p} \frac{(-1)^{j-1}}{j f^{j}(x)} \sum_{k=1}^{p} c_{k}(\boldsymbol{a}) \bar{D}_{\beta / a_{k}}^{j}(x) \\
\mathcal{R}_{\beta, p+1}(x)= & \frac{(-1)^{p}}{f^{p+1}(x)} \sum_{k=1}^{p} c_{k}(\boldsymbol{a}) \bar{D}_{\beta / a_{k}}^{p+1}(x) \int_{0}^{1} \frac{(1-\theta)^{p}}{\left(1+\theta \bar{D}_{\beta / a_{k}}(x) / f(x)\right)^{p+1}} d \theta \\
\mathcal{R}_{\beta, T S_{\boldsymbol{a}}^{p}}(x)= & f(x) \mathcal{R}_{\beta, p+1}(x) \exp \left\{\mathcal{Q}_{\beta, T S_{\boldsymbol{a}}^{p}}(x)\right\} \int_{0}^{1} \exp \left\{\theta \mathcal{R}_{\beta, p+1}(x)\right\} d \theta \\
& +\frac{f(x)}{p !} \mathcal{Q}_{\beta, T S_{\boldsymbol{a}}^{p}}^{p+1}(x) \int_{0}^{1} \exp \left\{\theta \mathcal{Q}_{\beta, T S_{\boldsymbol{a}}^{p}}(x)\right\}(1-\theta)^{p} d \theta
\end{aligned}
$$


For simplicity, we use the notation

$$
\mathcal{I}_{\beta, T S_{a}^{p}}(x)=\sum_{J=1}^{2} \mathcal{I}_{\beta, T S_{a}^{p}}^{[J]}(x)
$$

with

$$
\begin{aligned}
& \mathcal{I}_{\beta, T S_{\boldsymbol{a}}^{p}}^{[1]}(x)=\sum_{j=2}^{p} \frac{(-1)^{j-1}}{j f^{j-1}(x)} \sum_{k=1}^{p} c_{k}(\boldsymbol{a}) \bar{D}_{\beta / a_{k}}^{j}(x), \\
& \mathcal{I}_{\beta, T S_{\boldsymbol{a}}^{p}}^{[2]}(x)=\sum_{i=2}^{p} \frac{f(x)}{i !}\left[\sum_{j=1}^{p} \frac{(-1)^{j-1}}{j f^{j}(x)} \sum_{k=1}^{p} c_{k}(\boldsymbol{a}) \bar{D}_{\beta / a_{k}}^{j}(x)\right]^{i} .
\end{aligned}
$$

Note that $\sum_{k=1}^{p} c_{k}(\boldsymbol{a}) \bar{D}_{\beta / a_{k}}(x)=\widehat{f}_{\beta, A D D_{\boldsymbol{a}}^{p}}(x)-f(x)$, using (7). In summary, we have:

Lemma A.3 When $f(x)>0$, the stochastic expansion of $\widehat{f}_{\beta, T S_{\boldsymbol{a}}^{p}}(x)$ is given by

$$
\widehat{f}_{\beta, T S_{\boldsymbol{a}}^{p}}(x)=\widehat{f}_{\beta, A D D_{\boldsymbol{a}}^{p}}(x)+\mathcal{I}_{\beta, T S_{\boldsymbol{a}}^{p}}(x)+\mathcal{R}_{\beta, T S_{\boldsymbol{a}}^{p}}(x) .
$$

We prepare the following lemmas to prove Theorems $8-10$ and $8^{\prime}-10^{\prime}$ for the TS-type.

Lemma A.4 Under Assumption A1(i), we have

$$
0 \leq \widehat{f}_{\beta, T S_{\boldsymbol{a}}^{p}}(x) \leq \prod_{k=1}^{p}\left\{\left(C_{K} \frac{a_{k}}{\beta}+\frac{\epsilon}{a_{k}}\right)^{\chi_{\left\{c_{k}(\boldsymbol{a})>0\right\}} c_{k}(\boldsymbol{a})}\left(\frac{\epsilon}{a_{k}}\right)^{\chi_{\left\{c_{k}(\boldsymbol{a})<0\right\}} c_{k}(\boldsymbol{a})}\right\}=M_{\beta, T S_{\boldsymbol{a}}^{p}} \quad \text { (say). }
$$

Proof Use $0 \leq \widehat{f}_{\beta}(x) \leq C_{K} \beta^{-1}$ (see Assumption A1(i)).

Lemma A.5 $(\mathcal{S}=[0, \infty))$ Given $p \in \mathbb{N} \backslash\{1\}$ and $\left(\iota, \iota_{0}\right) \in \widetilde{I}_{p, 1}$, suppose that Assumptions A1(i) and $\mathrm{A} 6[p]_{\iota_{1}, \iota_{2}}$ hold for some constant $\left(\iota_{1}, \iota_{2}\right) \in I_{p,\left(\iota, \iota_{0}\right), T S}$, i.e.,

$$
0<\iota_{1}<\frac{1}{1+2 \iota_{0}}, \quad \iota_{2}>1+\left(\iota+\iota_{0}\right)(p-1)
$$

and let $x \in \mathcal{I}_{\iota, \iota_{0}}\left[r_{\beta}\right]$.

(i) In addition, suppose that Assumptions $\mathrm{A}^{\prime}[p]$ and $\mathrm{A} 5[p]$ (i) hold. Then,

$$
\begin{aligned}
E\left[\mathcal{I}_{\beta, T S_{\boldsymbol{a}}^{p}}^{[1]}(x)\right]= & \frac{(-1)^{p-1} \beta^{p}}{\prod_{k=1}^{p} a_{k}} \sum_{j=2}^{p} \frac{(-1)^{j-1}}{j f^{j-1}(x)} \sum_{\mathcal{L}_{p, j}} \prod_{m=1}^{j} \gamma_{\ell_{m}}(x ; f) \\
& +O\left(\beta^{p+1-\iota_{0}(p-1)}(1+x)^{p+1}+\beta^{p+\iota_{2}-1-\iota_{0}(p-1)}(1+x)^{p-1}+\beta^{-\iota_{0}} \sum_{k=1}^{p} V\left[\widehat{f}_{\beta / a_{k}}(x)\right]\right),
\end{aligned}
$$$$
E\left[\mathcal{I}_{\beta, T S_{a}^{p}}^{[2]}(x)\right]=O\left(\beta^{p+1-\iota_{0} p}(1+x)^{p+1}+\beta^{-\iota_{0}} \sum_{k=1}^{p} V\left[\widehat{f}_{\beta / a_{k}}(x)\right]\right),
$$

hence,

$$
\begin{aligned}
E\left[\mathcal{I}_{\beta, T S_{\boldsymbol{a}}^{p}}(x)\right]= & \frac{(-1)^{p-1} \beta^{p}}{\prod_{k=1}^{p} a_{k}} \sum_{j=2}^{p} \frac{(-1)^{j-1}}{j f^{j-1}(x)} \sum_{\mathcal{L}_{p, j}} \prod_{m=1}^{j} \gamma_{\ell_{m}}(x ; f) \\
& +O\left(\beta^{p+1-\iota_{0} p}(1+x)^{p+1}+\beta^{p+\iota_{2}-1-\iota_{0}(p-1)}(1+x)^{p-1}+\beta^{-\iota_{0}} \sum_{k=1}^{p} V\left[\widehat{f}_{\beta / a_{k}}(x)\right]\right) .
\end{aligned}
$$


(ii) On the other hand, suppose that Assumptions $\mathrm{A} 4^{\prime}[1]$ and $\mathrm{A} 5^{\prime}(\mathrm{i})$ hold. Then,

$$
\begin{aligned}
& V\left[\mathcal{I}_{\beta, T S_{\boldsymbol{a}}^{p}}^{[1]}(x)\right]=O\left(\left\{\beta^{2\left(1-\iota_{0}\right)}(1+x)^{2}+n^{-1} \beta^{-\left(1+2 \iota_{0}\right)}\right\} \sum_{k=1}^{p} V\left[\widehat{f}_{\beta / a_{k}}(x)\right]\right), \\
& V\left[\mathcal{I}_{\beta, T S_{\boldsymbol{a}}^{p}}^{[2]}(x)\right]=O\left(\left\{\beta^{2\left(1-\iota_{0}\right)}(1+x)^{2}+n^{-1} \beta^{-\left(1+2 \iota_{0}\right)}\right\} \sum_{k=1}^{p} V\left[\widehat{f}_{\beta / a_{k}}(x)\right]\right),
\end{aligned}
$$

hence,

$$
V\left[\mathcal{I}_{\beta, T S_{a}^{p}}(x)\right] \leq 2 \sum_{J=1}^{2} V\left[\mathcal{I}_{\beta, T S_{a}^{p}}^{[J]}(x)\right]=O\left(\left\{\beta^{2\left(1-\iota_{0}\right)}(1+x)^{2}+n^{-1} \beta^{-\left(1+2 \iota_{0}\right)}\right\} \sum_{k=1}^{p} V\left[\widehat{f}_{\beta / a_{k}}(x)\right]\right) .
$$

Also, for any $u \geq 1$,

$E\left[\left|\mathcal{R}_{\beta, T S_{\boldsymbol{a}}^{p}}(x)\right|^{u}\right]=O\left(\beta^{u\left(p+1-\iota_{0} p\right)}(1+x)^{u(p+1)}+\beta^{-\iota_{0}(2-u)}\left(n \beta^{1+2 \iota_{0}}\right)^{-\{u(p+1)-2\} / 2} \sum_{k=1}^{p} V\left[\widehat{f}_{\beta / a_{k}}(x)\right]\right)$.

Lemma A.5' $(\mathcal{S}=[0,1])$ Given $p \in \mathbb{N} \backslash\{1\}$, suppose that Assumptions A1(i) and A6 $[p]_{\iota_{1}, \iota_{2}}$ hold for some constant $\left(\iota_{1}, \iota_{2}\right) \in I_{p,(0,0), T S}$, i.e., $0<\iota_{1}<1$ and $\iota_{2}>1$, and let $x \in \mathcal{I}$.

(i) In addition, suppose that Assumptions $\mathrm{A}^{\prime}[p]$ and $\mathrm{A} 5[p]$ (i) hold. Then,

$$
\begin{aligned}
& E\left[\mathcal{I}_{\beta, T S_{\boldsymbol{a}}^{p}}^{[1]}(x)\right]=\frac{(-1)^{p-1} \beta^{p}}{\prod_{k=1}^{p} a_{k}} \sum_{j=2}^{p} \frac{(-1)^{j-1}}{j f^{j-1}(x)} \sum_{\mathcal{L}_{p, j}} \prod_{m=1}^{j} \gamma_{\ell_{m}}(x ; f)+O\left(\beta^{p+\min \left(1, \iota_{2}-1\right)}+\sum_{k=1}^{p} V\left[\widehat{f}_{\beta / a_{k}}(x)\right]\right), \\
& E\left[\mathcal{I}_{\beta, T S_{\boldsymbol{a}}^{p}}^{[2]}(x)\right]=O\left(\beta^{p+1}+\sum_{k=1}^{p} V\left[\widehat{f}_{\beta / a_{k}}(x)\right]\right),
\end{aligned}
$$

hence,

$E\left[\mathcal{I}_{\beta, T S_{\boldsymbol{a}}^{p}}(x)\right]=\frac{(-1)^{p-1} \beta^{p}}{\prod_{k=1}^{p} a_{k}} \sum_{j=2}^{p} \frac{(-1)^{j-1}}{j f^{j-1}(x)} \sum_{\mathcal{L}_{p, j}} \prod_{m=1}^{j} \gamma_{\ell_{m}}(x ; f)+O\left(\beta^{p+\min \left(1, \iota_{2}-1\right)}+\sum_{k=1}^{p} V\left[\widehat{f}_{\beta / a_{k}}(x)\right]\right)$.

(ii) On the other hand, suppose that Assumptions $\mathrm{A} 4^{\prime}[1]$ and $\mathrm{A} 5^{\prime}(\mathrm{i})$ hold. Then,

$$
\begin{aligned}
& V\left[\mathcal{I}_{\beta, T S_{\boldsymbol{a}}^{p}}^{[1]}(x)\right]=O\left(\left(\beta^{2}+n^{-1} \beta^{-1}\right) \sum_{k=1}^{p} V\left[\widehat{f}_{\beta / a_{k}}(x)\right]\right), \\
& V\left[\mathcal{I}_{\beta, T S_{\boldsymbol{a}}^{p}}^{[2]}(x)\right]=O\left(\left(\beta^{2}+n^{-1} \beta^{-1}\right) \sum_{k=1}^{p} V\left[\widehat{f}_{\beta / a_{k}}(x)\right]\right),
\end{aligned}
$$

hence,

$$
V\left[\mathcal{I}_{\beta, T S_{\boldsymbol{a}}^{p}}(x)\right] \leq 2 \sum_{J=1}^{2} V\left[\mathcal{I}_{\beta, T S_{\boldsymbol{a}}^{p}}^{[J]}(x)\right]=O\left(\left(\beta^{2}+n^{-1} \beta^{-1}\right) \sum_{k=1}^{p} V\left[\widehat{f}_{\beta / a_{k}}(x)\right]\right) .
$$

Also, for any $u \geq 1$,

$$
E\left[\left|\mathcal{R}_{\beta, T S_{\boldsymbol{a}}^{p}}(x)\right|^{u}\right]=O\left(\beta^{u(p+1)}+(n \beta)^{-\{u(p+1)-2\} / 2} \sum_{k=1}^{p} V\left[\widehat{f}_{\beta / a_{k}}(x)\right]\right) .
$$


The proofs of Lemmas A.5 and A.5' are in supplemental issue (Supplemental appendix to "Higher-order bias corrections for kernel type density estimators on the unit or semi-infinite interval").

\section{A.5.2. Stochastic expansion of JF-type estimator and auxiliary lemmas}

Write

$$
\bar{D}_{\beta}^{\dagger}(x)=\widehat{f}_{\beta}(x)+\epsilon-f(x) \quad \text { and } \quad \bar{D}_{\beta, A D D_{\boldsymbol{a}}^{p}}(x)=\widehat{f}_{\beta, A D D_{\boldsymbol{a}}^{p}}(x)-f(x) .
$$

Noting that, on the event $\left\{\widehat{f}_{\beta, A D D_{\boldsymbol{a}}^{p}}(x)>0\right\}$,

$$
\log \left\{\frac{\widehat{f}_{\beta, A D D_{a}^{p}}(x)}{\widehat{f}_{\beta}(x)+\epsilon}\right\}=\sum_{j=1}^{p} \frac{(-1)^{j-1}}{j}\left\{\frac{\widehat{f}_{\beta, A D D_{a}^{p}}(x)}{\widehat{f}_{\beta}(x)+\epsilon}-1\right\}^{j}-\mathcal{R}_{\beta, \mathrm{i}}(x),
$$

we have

$$
\begin{aligned}
\widehat{f}_{\beta, J F_{\boldsymbol{a}}^{p}}(x)= & \widehat{f}_{\beta, J F_{\boldsymbol{a}}^{p}}(x) \chi_{\left\{\widehat{f}_{\beta, A D D_{\boldsymbol{a}}^{p}}(x)>0\right\}}+\widehat{f}_{\beta, J F_{\boldsymbol{a}}^{p}}(x) \chi_{\left\{\widehat{f}_{\beta, A D D_{\boldsymbol{a}}^{p}}(x) \leq 0\right\}} \\
= & \widehat{f}_{\beta, A D D_{\boldsymbol{a}}^{p}}(x) \exp \left[\frac{(-1)^{p}}{p}\left\{\frac{\widehat{f}_{\beta, A D D_{\boldsymbol{a}}^{p}}(x)}{\widehat{f}_{\beta}(x)+\epsilon}-1\right\}^{p}\right]\left\{1+\mathcal{R}_{\beta, \mathrm{ii}}(x)\right\} \chi_{\left\{\widehat{f}_{\beta, A D D_{\boldsymbol{a}}^{p}}(x)>0\right\}} \\
& +\widehat{f}_{\beta, J F_{\boldsymbol{a}}^{p}}(x) \chi_{\left\{\widehat{f}_{\beta, A D D_{\boldsymbol{a}}^{p}}(x) \leq 0\right\}} \\
= & \widehat{f}_{\beta, A D D_{\boldsymbol{a}}^{p}}(x)\left[1+\frac{(-1)^{p}}{p}\left\{\frac{\widehat{f}_{\beta, A D D_{\boldsymbol{a}}^{p}}(x)}{\widehat{f}_{\beta}(x)+\epsilon}-1\right\}^{p}+\mathcal{R}_{\beta, \mathrm{iii}}(x)\right. \\
& \left.+\exp \left[\frac{(-1)^{p}}{p}\left\{\frac{\widehat{f}_{\beta, A D D_{\boldsymbol{a}}^{p}}(x)}{\widehat{f}_{\beta}(x)+\epsilon}-1\right\}^{p}\right] \mathcal{R}_{\beta, \mathrm{ii}}(x)\right] \chi_{\left\{\widehat{f}_{\beta, A D D_{\boldsymbol{a}}^{p}}(x)>0\right\}} \\
= & \widehat{f}_{\beta, A D D_{\boldsymbol{a}}^{p}}(x)+\frac{(-1)^{p}}{p} f(x)\left\{\frac{\widehat{f}_{\beta, A D D_{\boldsymbol{a}}^{p}}(x)}{\widehat{f}_{\beta}(x)+\epsilon}-1\right\}^{p}+\sum_{j=1}^{3} \widehat{\mathcal{R}}_{\beta, J F_{\boldsymbol{a}}^{p}}^{[j]}(x) \\
= & \widehat{f}_{\beta, A D D_{\boldsymbol{a}}^{p}}(x)+\frac{(-1)^{p}}{p f^{p-1}(x)}\left\{\bar{D}_{\beta, A D D_{a}^{p}}(x)-\bar{D}_{\beta}^{\dagger}(x)\right\}^{p}+\sum_{j=1}^{4} \mathcal{R}_{\beta, J F_{\boldsymbol{a}}^{p}}^{[j]}(x)
\end{aligned}
$$

(for the last equality, we assumed $f(x)>0$ ), where

$$
\begin{aligned}
\mathcal{R}_{\beta, \mathrm{i}}(x) & =(-1)^{p+1}\left\{\frac{\widehat{f}_{\beta, A D D_{a}^{p}}(x)}{\widehat{f}_{\beta}(x)+\epsilon}-1\right\}^{p+1} \int_{0}^{1}\left\{1-\theta+\frac{\theta \widehat{f}_{\beta, A D D_{a}^{p}}(x)}{\widehat{f}_{\beta}(x)+\epsilon}\right\}^{-(p+1)}(1-\theta)^{p} d \theta, \\
\mathcal{R}_{\beta, \mathrm{ii}}(x) & =\mathcal{R}_{\beta, \mathrm{i}}(x) \int_{0}^{1} \exp \left\{\theta \mathcal{R}_{\beta, \mathrm{i}}(x)\right\} d \theta, \\
\mathcal{R}_{\beta, \mathrm{iii}}(x) & =\frac{1}{p^{2}}\left\{\frac{\widehat{f}_{\beta, A D D_{a}^{p}}(x)}{\widehat{f}_{\beta}(x)+\epsilon}-1\right\}^{2 p} \int_{0}^{1} \exp \left[\theta \frac{(-1)^{p}}{p}\left\{\frac{\widehat{f}_{\beta, A D D_{a}^{p}}(x)}{\widehat{f}_{\beta}(x)+\epsilon}-1\right\}^{p}\right](1-\theta) d \theta, \\
\mathcal{R}_{\beta, J F_{\boldsymbol{a}}^{p}}^{[1]}(x) & =\left[\widehat{f}_{\beta, J F_{\boldsymbol{a}}^{p}}(x)-\widehat{f}_{\beta, A D D_{\boldsymbol{a}}^{p}}(x)-\frac{(-1)^{p}}{p} \widehat{f}_{\beta, A D D_{\boldsymbol{a}}^{p}}(x)\left\{\frac{\widehat{f}_{\beta, A D D_{a}^{p}}(x)}{\widehat{f}_{\beta}(x)+\epsilon}-1\right\}^{p}\right] \chi_{\left\{\widehat{f}_{\beta, A D D_{a}^{p}}(x) \leq 0\right\}}, \\
\mathcal{R}_{\beta, J F_{\boldsymbol{a}}^{p}}^{[2]}(x) & =\widehat{f}_{\beta, A D D_{\boldsymbol{a}}^{p}}(x)\left[\exp \left[\frac{(-1)^{p}}{p}\left\{\frac{\widehat{f}_{\beta, A D D_{\boldsymbol{a}}^{p}}(x)}{\widehat{f}_{\beta}(x)+\epsilon}-1\right\}^{p}\right] \mathcal{R}_{\beta, \mathrm{ii}}(x)+\mathcal{R}_{\beta, \mathrm{iii}}(x)\right] \chi_{\left\{\widehat{f}_{\beta, A D D_{a}^{p}}(x)>0\right\}},
\end{aligned}
$$




$$
\begin{aligned}
\mathcal{R}_{\beta, J F_{\boldsymbol{a}}^{p}}^{[3]}(x) & =\frac{(-1)^{p}}{p} \bar{D}_{\beta, A D D_{\boldsymbol{a}}^{p}(x)}\left\{\frac{\widehat{f}_{\beta, A D D_{a}^{p}}(x)}{\widehat{f}_{\beta}(x)+\epsilon}-1\right\}^{p}, \\
\mathcal{R}_{\beta, J F_{\boldsymbol{a}}^{p}}^{[4]}(x) & =\frac{(-1)^{p}}{p} f(x)\left\{\frac{\widehat{f}_{\beta, A D D_{a}^{p}}(x)}{\widehat{f}_{\beta}(x)+\epsilon}-1\right\}^{p}\left[1-\left\{1+\frac{\bar{D}_{\beta}^{\dagger}(x)}{f(x)}\right\}^{p}\right] \\
& =\frac{(-1)^{p-1}}{p} f(x)\left\{\frac{\widehat{f}_{\beta, A D D_{a}^{p}}(x)}{\widehat{f}_{\beta}(x)+\epsilon}-1\right\}^{p} \sum_{j=1}^{p}{ }_{p} C_{j}\left\{\frac{\bar{D}_{\beta}^{\dagger}(x)}{f(x)}\right\}^{j} .
\end{aligned}
$$

For simplicity, we use the notations

$$
\mathcal{I}_{\beta, J F_{a}^{p}}(x)=\frac{(-1)^{p}}{p f^{p-1}(x)}\left\{\bar{D}_{\beta, A D D_{a}^{p}}(x)-\bar{D}_{\beta}^{\dagger}(x)\right\}^{p} \quad \text { and } \quad \mathcal{R}_{\beta, J F_{a}^{p}}(x)=\sum_{j=1}^{4} \mathcal{R}_{\beta, J F_{a}^{p}}^{[j]}(x) .
$$

In summary, we have:

Lemma A.6 When $f(x)>0$, the stochastic expansion of $\widehat{f}_{\beta, J F_{\boldsymbol{a}}^{p}}(x)$ is given by

$$
\widehat{f}_{\beta, J F_{\boldsymbol{a}}^{p}}(x)=\widehat{f}_{\beta, A D D_{\boldsymbol{a}}^{p}}(x)+\mathcal{I}_{\beta, J F_{\boldsymbol{a}}^{p}}(x)+\mathcal{R}_{\beta, J F_{\boldsymbol{a}}^{p}}(x) .
$$

We prepare the following lemmas to prove Theorems $8-10$ and $8^{\prime}-10^{\prime}$ for the JF-type.

Lemma A.7 Under Assumption A1(i), we have

$$
0 \leq \widehat{f}_{\beta, J F_{a}^{p}}(x) \leq M_{\beta, J F_{a}^{p}}
$$

where

$$
M_{\beta, J F_{a}^{p}}= \begin{cases}\left(C_{K} \beta^{-1}+\epsilon\right) \exp \left\{c_{1}(\boldsymbol{a})\right\}, & p=2 \text { and } 0<a_{2}<a_{1}=1, \\ \left(C_{K} \beta^{-1}+\epsilon\right) \exp \left\{\sum_{k=1}^{p}\left|c_{k}(\boldsymbol{a})\right| C_{K} a_{k}(\beta \epsilon)^{-1}+1\right\}, & p=2 \text { and } 1=a_{1}<a_{2}, \\ \left(C_{K} \beta^{-1}+\epsilon\right) \exp \left[\frac{c_{p}+1}{2}\left\{\sum_{k=1}^{p}\left|c_{k}(\boldsymbol{a})\right| C_{K} a_{k}(\beta \epsilon)^{-1}+1\right\}^{c_{p}}\right], & p>2,\end{cases}
$$

with

$$
c_{p}=\left\{\begin{array}{l}
p-1, p(>2) \text { is even } \\
p-2, p(>2) \text { is odd }
\end{array}\right.
$$

Proof Use $0 \leq \widehat{f}_{\beta}(x) \leq C_{K} \beta^{-1}$ (see Assumption A1(i)) to bound

$$
0 \leq \widehat{f}_{\beta, J F_{\boldsymbol{a}}^{p}}(x) \leq\left\{\widehat{f}_{\beta}(x)+\epsilon\right\} \exp \left[\sum_{j=1}^{p-1} \chi_{\{j \text { is odd }\}} \frac{1}{j}\left\{\frac{\left|\widehat{f}_{\beta, A D D_{\boldsymbol{a}}^{p}}(x)\right|}{\widehat{f}_{\beta}(x)+\epsilon}+1\right\}^{j}\right] .
$$

Exceptionally, if $p=2$ and $0<a_{2}<a_{1}=1$, then, $c_{1}(\boldsymbol{a})>0$ and $c_{2}(\boldsymbol{a})<0$, hence,

$$
0 \leq \widehat{f}_{\beta, J F_{\boldsymbol{a}}^{p}}(x) \leq\left\{\widehat{f}_{\beta}(x)+\epsilon\right\} \exp \left\{c_{1}(\boldsymbol{a})\right\}
$$


Lemma A.8 $(\mathcal{S}=[0, \infty))$ Given $p \in \mathbb{N} \backslash\{1\}$ and $\left(\iota, \iota_{0}\right) \in \widetilde{I}_{p, 1}$, suppose that Assumptions A1(i) and $\mathrm{A} 6[p]_{\iota_{1}, \iota_{2}}$ hold for some constant $\left(\iota_{1}, \iota_{2}\right) \in I_{p,\left(\iota, \iota_{0}\right), J F}$, i.e.,

$$
0<\iota_{1}<\frac{1}{1+2 \iota_{0}+c_{p, J F}\left(1+\iota_{2}\right)}, \quad \iota_{2}>1+\left(\iota+\iota_{0}\right)(p-1),
$$

and let $x \in \mathcal{I}_{\iota, \iota_{0}}\left[r_{\beta}\right]$.

(i) In addition, suppose that Assumptions A4'[2] and A5[2](i) hold. Then,

$$
\begin{aligned}
& E\left[\mathcal{I}_{\left.\beta, J F_{a}^{p}(x)\right]}\right. \\
& =\beta^{p} \frac{\gamma_{1}^{p}(x ; f)}{p f^{p-1}(x)}+O\left(\beta^{p+1-\iota_{0}(p-1)}(1+x)^{p+1}+\beta^{p+\iota_{2}-1-\iota_{0}(p-1)}(1+x)^{p-1}+\beta^{-\iota_{0}} \sum_{k=1}^{p} V\left[\widehat{f}_{\beta / a_{k}}(x)\right]\right) .
\end{aligned}
$$

(ii) On the other hand, suppose that Assumptions A4'[1] and A5'(i) hold. Then,

$$
V\left[\mathcal{I}_{\beta, J F_{a}^{p}}(x)\right]=O\left(\left\{\beta^{2\left(1-\iota_{0}\right)}(1+x)^{2}+n^{-1} \beta^{-\left(1+2 \iota_{0}\right)}\right\} \sum_{k=1}^{p} V\left[\widehat{f}_{\beta / a_{k}}(x)\right]\right) .
$$

Also, for any $u \geq 1$,

$$
\begin{aligned}
& E\left[\mid \mathcal{R}_{\beta,\left.J F_{a}^{p}(x)\right|^{u}}\right. \\
& =O\left(\beta^{u\left(p+1-\iota_{0} p\right)}(1+x)^{u(p+1)}+\beta^{-\iota_{0}(2-u)}\left(n \beta^{1+2 \iota_{0}}\right)^{-\{u(p+1)-2\} / 2} \sum_{k=1}^{p} V\left[\widehat{f}_{\beta / a_{k}}(x)\right]\right) .
\end{aligned}
$$

Lemma A.8 $(\mathcal{S}=[0,1])$ Given $p \in \mathbb{N} \backslash\{1\}$, suppose that Assumptions A1(i) and A6 $[p]_{\iota_{1}, \iota_{2}}$ hold for some constant $\left(\iota_{1}, \iota_{2}\right) \in I_{p,(0,0), J F}$, i.e.,

$$
0<\iota_{1}<\frac{1}{1+c_{p, J F}\left(1+\iota_{2}\right)}, \quad \iota_{2}>1
$$

and let $x \in \mathcal{I}$.

(i) In addition, suppose that Assumptions $\mathrm{A}^{\prime}[2]$ and $\mathrm{A} 5[2](\mathrm{i})$ hold. Then,

$$
E\left[\mathcal{I}_{\beta, J F_{a}^{p}}(x)\right]=\beta^{p} \frac{\gamma_{1}^{p}(x ; f)}{p f^{p-1}(x)}+O\left(\beta^{p+\min \left(1, \iota_{2}-1\right)}+\sum_{k=1}^{p} V\left[\widehat{f}_{\beta / a_{k}}(x)\right]\right) .
$$

(ii) On the other hand, suppose that Assumptions $\mathrm{A} 4^{\prime}[1]$ and $\mathrm{A} 5^{\prime}(\mathrm{i})$ hold. Then,

$$
V\left[\mathcal{I}_{\beta, J F_{a}^{p}}(x)\right]=O\left(\left(\beta^{2}+n^{-1} \beta^{-1}\right) \sum_{k=1}^{p} V\left[\widehat{f}_{\beta / a_{k}}(x)\right]\right) .
$$

Also, for any $u \geq 1$,

$$
E\left[\left|\mathcal{R}_{\beta, J F_{a}^{p}}(x)\right|^{u}\right]=O\left(\beta^{u(p+1)}+(n \beta)^{-\{u(p+1)-2\} / 2} \sum_{k=1}^{p} V\left[\widehat{f}_{\beta / a_{k}}(x)\right]\right) .
$$

The proofs of Lemmas A.8 and A.8' are in supplemental issue (Supplemental appendix to "Higher-order bias corrections for kernel type density estimators on the unit or semi-infinite interval"). 


\section{A.5.3. Proofs of Theorems $8-10$ and $8^{\prime}-10^{\prime}$}

Assuming $f(x)>0$, Lemma A.3 (or A.6) yields

$$
\begin{aligned}
E\left[\widehat{f}_{\beta, \#_{\boldsymbol{a}}^{p}}(x)\right]= & E\left[\widehat{f}_{\beta, A D D_{\boldsymbol{a}}^{p}}(x)\right]+E\left[\mathcal{I}_{\beta, \#_{\boldsymbol{a}}^{p}}(x)\right]+E\left[\mathcal{R}_{\beta, \#_{\boldsymbol{a}}^{p}}(x)\right], \\
V\left[\widehat{f}_{\beta, \#_{\boldsymbol{a}}^{p}}(x)\right]= & V\left[\widehat{f}_{\beta, A D D_{\boldsymbol{a}}^{p}}(x)\right]+V\left[\mathcal{I}_{\beta, \#_{\boldsymbol{a}}^{p}}(x)+\mathcal{R}_{\beta, \#_{\boldsymbol{a}}^{p}}(x)\right] \\
& +2 \operatorname{Cov}\left[\widehat{f}_{\beta, A D D_{\boldsymbol{a}}^{p}}(x), \mathcal{I}_{\beta, \#_{\boldsymbol{a}}^{p}}(x)+\mathcal{R}_{\beta, \#_{\boldsymbol{a}}^{p}}(x)\right],
\end{aligned}
$$

where

$$
\begin{aligned}
V\left[\mathcal{I}_{\beta, \#_{\boldsymbol{a}}^{p}}(x)+\mathcal{R}_{\beta, \#_{\boldsymbol{a}}^{p}}(x)\right] & \leq 2\left\{V\left[\mathcal{I}_{\beta, \#_{\boldsymbol{a}}^{p}}(x)\right]+V\left[\mathcal{R}_{\beta, \#_{\boldsymbol{a}}^{p}}(x)\right]\right\} \\
& \leq 2\left\{V\left[\mathcal{I}_{\beta, \#_{\boldsymbol{a}}^{p}}(x)\right]+E\left[\mathcal{R}_{\beta, \#_{\boldsymbol{a}}^{p}}^{2}(x)\right]\right\}, \\
\left|\operatorname{Cov}\left[\widehat{f}_{\beta, A D D_{\boldsymbol{a}}^{p}}(x), \mathcal{I}_{\beta, \#_{\boldsymbol{a}}^{p}}(x)+\mathcal{R}_{\beta, \#_{\boldsymbol{a}}^{p}}(x)\right]\right| & \leq\left\{V\left[\widehat{f}_{\beta, A D D_{\boldsymbol{a}}^{p}}(x)\right] V\left[\mathcal{I}_{\beta, \#_{\boldsymbol{a}}^{p}}(x)+\mathcal{R}_{\beta, \#_{\boldsymbol{a}}^{p}}(x)\right]\right\}^{1 / 2}
\end{aligned}
$$

with $V\left[\widehat{f}_{\beta, A D D_{a}^{p}}(x)\right]=O\left(\sum_{k=1}^{p} V\left[\widehat{f}_{\beta / a_{k}}(x)\right]\right)$.

We are ready to prove Theorems $8-10$ and $8^{\prime}-10^{\prime}$.

Proof of Theorem 8 (i) Given $p \in \mathbb{N} \backslash\{1\}$ and $\left(\iota, \iota_{0}\right) \in \widetilde{I}_{p, \eta_{2 p}}\left(\subset \widetilde{I}_{p, 1}\right)$, where $\eta_{2 p} \in(0,1]$ is given in Assumption A5[p](ii), suppose that Assumption A6[p] $]_{\iota_{1}, \iota_{2}}$ holds for some constant

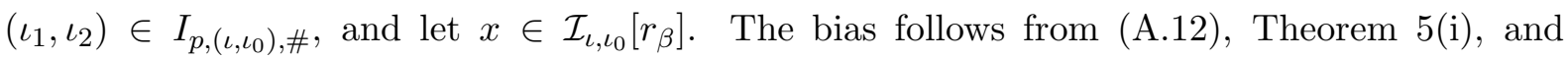
Lemmas A.5 (or A.8).

(ii) Given $p \in \mathbb{N} \backslash\{1\}$ and $\left(\iota, \iota_{0}\right) \in \widetilde{I}_{p, 1}$, suppose that Assumption A6[p] $]_{\iota_{1}, \iota_{2}}$ holds for some

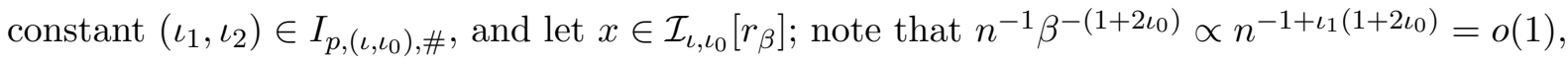
and that $r_{\beta}=O\left(\beta^{-\iota}\right)$ implies $\beta^{1-\iota_{0} p}\left(1+r_{\beta}\right)^{p+1}=O\left(\beta^{1-\iota_{0} p-(p+1) \iota}\right)=o(1)$. The variance follows from (A.13) and Lemma A.5(ii) (or A.8(ii)), i.e.,

$$
\begin{aligned}
& V\left[\mathcal{I}_{\beta, \#_{\boldsymbol{a}}^{p}}(x)+\mathcal{R}_{\beta, \#_{\boldsymbol{a}}^{p}}(x)\right] \\
& =O\left(\beta^{2\left(p+1-\iota_{0} p\right)}(1+x)^{2(p+1)}+\left\{\beta^{2\left(1-\iota_{0}\right)}(1+x)^{2}+n^{-1} \beta^{-\left(1+2 \iota_{0}\right)}\right\} \sum_{k=1}^{p} V\left[\widehat{f}_{\beta / a_{k}}(x)\right]\right)
\end{aligned}
$$

and

$$
\begin{aligned}
& \left|\operatorname{Cov}\left[\widehat{f}_{\beta, A D D_{\boldsymbol{a}}^{p}}(x), \mathcal{I}_{\beta, \#_{\boldsymbol{a}}^{p}}(x)+\mathcal{R}_{\beta, \#_{\boldsymbol{a}}^{p}}(x)\right]\right| \\
& =O\left(\beta^{p+1-\iota_{0} p}(1+x)^{p+1}\left(\sum_{k=1}^{p} V\left[\widehat{f}_{\beta / a_{k}}(x)\right]\right)^{1 / 2}+\left\{\beta^{1-\iota_{0}}(1+x)+n^{-1 / 2} \beta^{-\left(1 / 2+\iota_{0}\right)}\right\} \sum_{k=1}^{p} V\left[\widehat{f}_{\beta / a_{k}}(x)\right]\right) \\
& =O\left(\beta^{2 p+1-\iota_{0} p}(1+x)^{p+1}+\left\{\beta^{1-\iota_{0} p}(1+x)^{p+1}+n^{-1 / 2} \beta^{-\left(1 / 2+\iota_{0}\right)}\right\} \sum_{k=1}^{p} V\left[\widehat{f}_{\beta / a_{k}}(x)\right]\right) . \quad \square
\end{aligned}
$$

Proof of Theorem 9 Recall Lemma A.3 (or A.6). Under Assumption A6 $[p]_{\iota_{1}, \iota_{2}}$ for some 
constant $\left(\iota_{1}, \iota_{2}\right) \in I_{p, 2, \#}$ for $x \in \mathcal{S}_{I}$ or $\left(\iota_{1}, \iota_{2}\right) \in I_{p, 1, \#}$ for $x \in \mathcal{S}_{B}$, we have

$$
\begin{aligned}
& \left(n \beta^{1 / 2}\right)^{1 / 2}\left\{\widehat{f}_{\beta, \#_{\boldsymbol{a}}^{p}}(x)-E\left[\widehat{f}_{\beta, \#_{\boldsymbol{a}}^{p}}(x)\right]\right\} \\
& =\left(n \beta^{1 / 2}\right)^{1 / 2}\left\{\widehat{f}_{\beta, A D D_{\boldsymbol{a}}^{p}}(x)-E\left[\widehat{f}_{\beta, A D D_{\boldsymbol{a}}^{p}}(x)\right]\right\}+o_{p}(1) \quad \text { for fixed } x \in \mathcal{I}_{0,0}[r] \cap \mathcal{S}_{I}, \\
& (n \beta)^{1 / 2}\left\{\widehat{f}_{\beta, \#_{\boldsymbol{a}}^{p}}(x)-E\left[\widehat{f}_{\beta, \#_{\boldsymbol{a}}^{p}}(x)\right]\right\} \\
& =(n \beta)^{1 / 2}\left\{\widehat{f}_{\beta, A D D_{\boldsymbol{a}}^{p}}(x)-E\left[\widehat{f}_{\beta, A D D_{\boldsymbol{a}}^{p}}(x)\right]\right\}+o_{p}(1) \quad \text { for } x \in \mathcal{I}_{0,0}[r] \cap \mathcal{S}_{B},
\end{aligned}
$$

since, from $(\mathrm{A} .14)\left(\right.$ set $\left(\iota, \iota_{0}\right)=(0,0)$ and $\left.r_{\beta} \equiv r\right)$,

$$
\begin{aligned}
& n \beta^{1 / 2} V\left[\mathcal{I}_{\beta, \#_{\boldsymbol{a}}^{p}}(x)+\mathcal{R}_{\beta, \#_{\boldsymbol{a}}^{p}}(x)\right]=o(1) \quad \text { for fixed } x \in \mathcal{I}_{0,0}[r] \cap \mathcal{S}_{I}, \\
& n \beta V\left[\mathcal{I}_{\beta, \#_{\boldsymbol{a}}^{p}}(x)+\mathcal{R}_{\beta, \#_{\boldsymbol{a}}^{p}}(x)\right]=o(1) \quad \text { for } x \in \mathcal{I}_{0,0}[r] \cap \mathcal{S}_{B} .
\end{aligned}
$$

This, together with Theorem 6 , yields the results.

Proof of Theorem 10 Using Lemma A.4 (or A.7), we have

$$
\int_{r_{\beta}}^{\infty} w(x) E\left[\left\{\widehat{f}_{\beta, \#_{\boldsymbol{a}}^{p}}(x)-f(x)\right\}^{2}\right] d x \leq\left(M_{\beta, \#_{\boldsymbol{a}}^{p}}+\|f\|_{[0, \infty)}\right)^{2} \int_{r_{\beta}}^{\infty} w(x) d x=o\left(\beta^{2 p}\right),
$$

hence,

$$
\operatorname{MISE}\left[\widehat{f}_{\beta, \#_{a}^{p}} ; w\right]=\int_{0}^{r_{\beta}} w(x)\left[\left\{\operatorname{Bias}\left[\widehat{f}_{\beta, \#_{a}^{p}}(x)\right]\right\}^{2}+V\left[\widehat{f}_{\beta, \#_{a}^{p}}(x)\right]\right] d x+o\left(\beta^{2 p}\right) .
$$

Given $p \in \mathbb{N} \backslash\{1\}$ and $\left(\iota, \iota_{0}\right) \in \widetilde{I}_{p, \eta_{2 p}}\left(\subset \widetilde{I}_{p, 1}\right)$, where $\eta_{2 p} \in(0,1]$ is given in Assumption A5[p](ii),

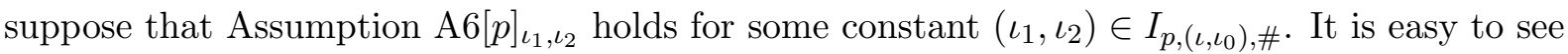
that $\int_{0}^{r_{\beta}} w(x) \mathcal{E}_{\beta, \#_{\boldsymbol{a}}^{p}}^{2}(x) d x=o\left(\beta^{2 p}+n^{-1} \beta^{-1 / 2}\right)$, since, for $x \in\left[0, r_{\beta}\right]$,

$$
w(x) \mathcal{E}_{\beta, \#_{\boldsymbol{a}}^{p}}^{2}(x)=O\left(\beta^{2 p} \omega_{\beta}^{2}\left(r_{\beta}\right) w(x)+n^{-1} \beta^{-\left(1+2 \iota_{0}\right)} \sum_{k=1}^{p} V\left[\widehat{f}_{\beta / a_{k}}(x)\right]\right)
$$

(we used $\omega_{\beta}\left(r_{\beta}\right)=o(1)$ and $n^{-1} \beta^{-\left(1+2 \iota_{0}\right)} \propto n^{-1+\iota_{1}\left(1+2 \iota_{0}\right)}=o(1)$ ). Then, Theorem 8(i) yields

$$
\begin{aligned}
& \left|\int_{0}^{r_{\beta}} w(x)\left\{\operatorname{Bias}\left[\widehat{f}_{\beta, \#_{\boldsymbol{a}}^{p}}(x)\right]\right\}^{2} d x-\beta^{2 p} \int_{0}^{\infty} w(x) B_{\#_{\boldsymbol{a}}^{p}}^{2}(x ; f) d x\right| \\
& \leq 2 \beta^{p}\left\{\int_{0}^{\infty} w(x) B_{\#_{\boldsymbol{a}}^{p}}^{2}(x ; f) d x \int_{0}^{r_{\beta}} w(x) \mathcal{E}_{\beta, \#_{\boldsymbol{a}}^{p}}^{2}(x) d x\right\}^{1 / 2}+\int_{0}^{r_{\beta}} w(x) \mathcal{E}_{\beta, \#_{\boldsymbol{a}}^{p}}^{2}(x) d x \\
& \quad+\beta^{2 p} \int_{r_{\beta}}^{\infty} w(x) B_{\#_{\boldsymbol{a}}^{p}}^{2}(x ; f) d x \\
& =o\left(\beta^{2 p}+n^{-1} \beta^{-1 / 2}\right),
\end{aligned}
$$

whereas, Theorem 8(ii) yields

$$
\begin{aligned}
& \int_{0}^{r_{\beta}} w(x) V\left[\widehat{f}_{\beta, \#_{\boldsymbol{a}}^{p}}(x)\right] d x \\
& =\int_{0}^{r_{\beta}} w(x) V\left[\widehat{f}_{\beta, A D D_{\boldsymbol{a}}^{p}}(x)\right] d x+o\left(\beta^{2 p}\right) \int_{0}^{\infty} w(x) d x+o(1) \int_{0}^{\infty} \sum_{k=1}^{p} V\left[\widehat{f}_{\beta / a_{k}}(x)\right] d x \\
& =\int_{0}^{r_{\beta}} w(x) V\left[\widehat{f}_{\beta, A D D_{\boldsymbol{a}}^{p}}(x)\right] d x+o\left(\beta^{2 p}+n^{-1} \beta^{-1 / 2}\right) .
\end{aligned}
$$


With a slight modification of Proof of Lemma A.2(ii) (recall (A.3)), we can show that, choosing $\tau \in(1 / 2,1)$,

$$
\begin{aligned}
& \left|\int_{0}^{r_{\beta}} w(x) V\left[\widehat{f}_{\beta, A D D_{a}^{p}}(x)\right] d x-n^{-1} \beta^{-1 / 2} \lambda_{p, \boldsymbol{a}} \int_{0}^{\infty} w(x) V(x ; f) d x\right| \\
& \leq\|w\|_{[0, \infty)} \sum_{j=1}^{p} \sum_{j^{\prime}=1}^{p}\left|c_{j}(\boldsymbol{a}) \| c_{j^{\prime}}(\boldsymbol{a})\right| \\
& \times\left[\left|\int_{0}^{\beta^{\tau}} \operatorname{Cov}\left[\widehat{f}_{\beta / a_{j}}(x), \widehat{f}_{\beta / a_{j}^{\prime}}(x)\right] d x\right|\right. \\
& \left.\quad+\int_{\beta^{\tau}}^{r_{\beta}}\left|\operatorname{Cov}\left[\widehat{f}_{\beta / a_{j}}(x), \widehat{f}_{\beta / a_{j}^{\prime}}(x)\right]-n^{-1} \beta^{-1 / 2}\left(\frac{2 a_{j} a_{j^{\prime}}}{a_{j}+a_{j^{\prime}}}\right)^{1 / 2} V(x ; f)\right| d x\right] \\
& \quad+n^{-1} \beta^{-1 / 2} \lambda_{p, \boldsymbol{a}} \frac{\|f\|_{[0, \infty)}}{2 \sqrt{\pi}}\left\{\|w\|_{[0, \infty)} \int_{0}^{\beta^{\tau}} \frac{1}{\sqrt{\psi(x)}} d x+\frac{1}{\sqrt{\psi\left(r_{\beta}\right)}} \int_{r_{\beta}}^{\infty} w(x) d x\right\} \\
& =o\left(n^{-1} \beta^{-1 / 2}\right), \quad
\end{aligned}
$$

using

$$
\begin{aligned}
n^{-1} \int_{\beta^{\tau}}^{r_{\beta}} w(x)\left\{\beta^{1 / 2} \frac{f(x)}{\sqrt{\psi^{3}(x)}}+1\right\} d x & \leq n^{-1}\left\{\|w\|_{[0, \infty)} \beta^{1 / 2-\tau} \int_{0}^{\infty} \frac{f(x)}{\sqrt{\psi(x)}} d x+\int_{0}^{\infty} w(x) d x\right\} \\
& =o\left(n^{-1} \beta^{-1 / 2}\right) .
\end{aligned}
$$

Proof of Theorem $\mathbf{8}^{\prime}$ Given $p \in \mathbb{N} \backslash\{1\}$, suppose that Assumption A6[p] $]_{\iota_{1}, \iota_{2}}$ holds for some

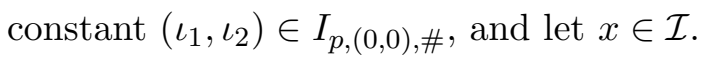

(i) The bias follows from (A.12), Theorem 5(i), and Lemmas A.5' (or A.8').

(ii) Noting that $n^{-1} \beta^{-1} \propto n^{-1+\iota_{1}}=o(1)$, the variance follows from (A.13) and Lemma A.5'(ii) (or A. $8^{\prime}(\mathrm{ii})$ ), i.e.,

$$
V\left[\mathcal{I}_{\beta, \# \boldsymbol{a}}^{p}(x)+\mathcal{R}_{\beta, \# \boldsymbol{a}}^{p}(x)\right]=O\left(\beta^{2(p+1)}+\left(\beta^{2}+n^{-1} \beta^{-1}\right) \sum_{k=1}^{p} V\left[\widehat{f}_{\beta / a_{k}}(x)\right]\right)
$$

and

$$
\begin{aligned}
& \left|\operatorname{Cov}\left[\widehat{f}_{\beta, A D D_{a}^{p}}(x), \mathcal{I}_{\beta, \#_{a}^{p}}(x)+\mathcal{R}_{\beta, \#_{a}^{p}}(x)\right]\right| \\
& =O\left(\beta^{p+1}\left(\sum_{k=1}^{p} V\left[\widehat{f}_{\beta / a_{k}}(x)\right]\right)^{1 / 2}+\left(\beta+n^{-1 / 2} \beta^{-1 / 2}\right) \sum_{k=1}^{p} V\left[\widehat{f}_{\beta / a_{k}}(x)\right]\right) \\
& =O\left(\beta^{2 p+1}+\left(\beta+n^{-1 / 2} \beta^{-1 / 2}\right) \sum_{k=1}^{p} V\left[\widehat{f}_{\beta / a_{k}}(x)\right]\right) .
\end{aligned}
$$

Proof of Theorem 9' Recall Lemma A.3 (or A.6). Under Assumption A6 $[p]_{\iota_{1}, \iota_{2}}$ for some constant $\left(\iota_{1}, \iota_{2}\right) \in I_{p, 2, \#}$ for $x \in \mathcal{S}_{I}$ or $\left(\iota_{1}, \iota_{2}\right) \in I_{p, 1, \#}$ for $x \in \mathcal{S}_{B}$, we have

$$
\begin{aligned}
& \left(n \beta^{1 / 2}\right)^{1 / 2}\left\{\widehat{f}_{\beta, \#_{\boldsymbol{a}}^{p}}(x)-E\left[\widehat{f}_{\beta, \#_{\boldsymbol{a}}^{p}}(x)\right]\right\} \\
& =\left(n \beta^{1 / 2}\right)^{1 / 2}\left\{\widehat{f}_{\beta, A D D_{\boldsymbol{a}}^{p}}(x)-E\left[\widehat{f}_{\beta, A D D_{\boldsymbol{a}}^{p}}(x)\right]\right\}+o_{p}(1) \quad \text { for fixed } x \in \mathcal{I} \cap \mathcal{S}_{I},
\end{aligned}
$$




$$
\begin{aligned}
& (n \beta)^{1 / 2}\left\{\widehat{f}_{\beta, \#_{\boldsymbol{a}}^{p}}(x)-E\left[\widehat{f}_{\beta, \#_{\boldsymbol{a}}^{p}}(x)\right]\right\} \\
& =(n \beta)^{1 / 2}\left\{\widehat{f}_{\beta, A D D_{\boldsymbol{a}}^{p}}(x)-E\left[\widehat{f}_{\beta, A D D_{\boldsymbol{a}}^{p}}(x)\right]\right\}+o_{p}(1) \text { for } x \in \mathcal{I} \cap \mathcal{S}_{B},
\end{aligned}
$$

since, from (A.14'),

$$
\begin{array}{cl}
n \beta^{1 / 2} V\left[\mathcal{I}_{\beta, \#_{\boldsymbol{a}}^{p}}(x)+\mathcal{R}_{\beta, \#_{\boldsymbol{a}}^{p}}(x)\right]=o(1) & \text { for fixed } x \in \mathcal{I} \cap \mathcal{S}_{I}, \\
n \beta V\left[\mathcal{I}_{\beta, \#_{\boldsymbol{a}}^{p}}(x)+\mathcal{R}_{\beta, \#_{\boldsymbol{a}}^{p}}(x)\right]=o(1) & \text { for } x \in \mathcal{I} \cap \mathcal{S}_{B} .
\end{array}
$$

This, together with Theorem 6 , yields the results.

Proof of Theorem 10' Given $p \in \mathbb{N} \backslash\{1\}$, suppose that Assumption A6 $[p]_{\iota_{1}, \iota_{2}}$ holds for some

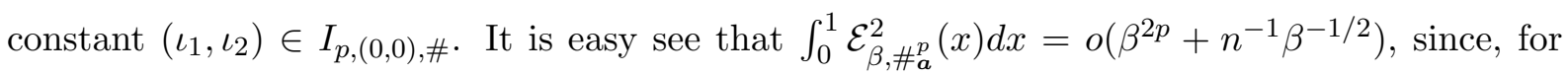
$x \in[0,1]$,

$$
\mathcal{E}_{\beta, \# \boldsymbol{a}}^{2}(x)=O\left(\beta^{2 p+\min \left\{\eta_{2 p}, 2\left(\iota_{2}-1\right)\right\}}+n^{-1} \beta^{-1} \sum_{k=1}^{p} V\left[\widehat{f}_{\beta / a_{k}}(x)\right]\right)
$$

(we used $n^{-1} \beta^{-1} \propto n^{-1+\iota_{1}}=o(1)$ ). Then, Theorem $8^{\prime}(\mathrm{i})$ yields

$$
\begin{aligned}
& \left|\int_{0}^{1}\left\{\operatorname{Bias}\left[\widehat{f}_{\beta, \#_{\boldsymbol{a}}^{p}}(x)\right]\right\}^{2} d x-\beta^{2 p} \int_{0}^{1} B_{\#_{\boldsymbol{a}}^{p}}^{2}(x ; f) d x\right| \\
& \leq 2 \beta^{p}\left\{\int_{0}^{1} B_{\#_{\boldsymbol{a}}^{p}}^{2}(x ; f) d x \int_{0}^{1} \mathcal{E}_{\beta, \#_{\boldsymbol{a}}^{p}}^{2}(x) d x\right\}^{1 / 2}+\int_{0}^{1} \mathcal{E}_{\beta, \#_{\boldsymbol{a}}^{p}}^{2}(x) d x=o\left(\beta^{2 p}+n^{-1} \beta^{-1 / 2}\right),
\end{aligned}
$$

whereas, Theorem $8^{\prime}($ ii) yields

$$
\begin{aligned}
\int_{0}^{1} V\left[\widehat{f}_{\beta, \# \boldsymbol{a}}^{p}(x)\right] d x & =\int_{0}^{1} V\left[\widehat{f}_{\beta, A D D_{\boldsymbol{a}}^{p}}(x)\right] d x+o\left(\beta^{2 p}\right)+o(1) \int_{0}^{1} \sum_{k=1}^{p} V\left[\widehat{f}_{\beta / a_{k}}(x)\right] d x \\
& =\int_{0}^{1} V\left[\widehat{f}_{\beta, A D D_{\boldsymbol{a}}^{p}}(x)\right] d x+o\left(\beta^{2 p}+n^{-1} \beta^{-1 / 2}\right) .
\end{aligned}
$$

With a slight modification of Proof of Lemma A.2(ii) (recall (A.3)), we can show that, choosing $\tau \in(1 / 2,1)$,

$$
\begin{aligned}
& \left|\int_{0}^{1} V\left[\widehat{f}_{\beta, A D D_{\boldsymbol{a}}^{p}}(x)\right] d x-n^{-1} \beta^{-1 / 2} \lambda_{p, \boldsymbol{a}} \int_{0}^{1} V(x ; f) d x\right| \\
& \leq \sum_{j=1}^{p} \sum_{j^{\prime}=1}^{p}\left|c_{j}(\boldsymbol{a})\right|\left|c_{j^{\prime}}(\boldsymbol{a})\right|\left[\left|\left(\int_{0}^{\beta^{\tau}}+\int_{1-\beta^{\tau}}^{1}\right) \operatorname{Cov}\left[\widehat{f}_{\beta / a_{j}}(x), \widehat{f}_{\beta / a_{j}^{\prime}}(x)\right] d x\right|\right. \\
& \left.+\int_{\beta^{\tau}}^{1-\beta^{\tau}}\left|\operatorname{Cov}\left[\widehat{f}_{\beta / a_{j}}(x), \widehat{f}_{\beta / a_{j}^{\prime}}(x)\right]-n^{-1} \beta^{-1 / 2}\left(\frac{2 a_{j} a_{j^{\prime}}}{a_{j}+a_{j^{\prime}}}\right)^{1 / 2} V(x ; f)\right| d x\right] \\
& +n^{-1} \beta^{-1 / 2} \lambda_{p, \boldsymbol{a}} \frac{\|f\|_{[0,1]}}{2 \sqrt{\pi}}\left(\int_{0}^{\beta^{\tau}}+\int_{1-\beta^{\tau}}^{1}\right) \frac{1}{\sqrt{\psi(x)}} d x \\
& =o\left(n^{-1} \beta^{-1 / 2}\right) \text {, }
\end{aligned}
$$

using

$$
n^{-1} \int_{\beta^{\tau}}^{1-\beta^{\tau}}\left\{\frac{\beta^{1 / 2}}{\sqrt{\psi^{3}(x)}}+1\right\} d x \leq n^{-1}\left\{\frac{\beta^{1 / 2}}{\beta^{\tau}\left(1-\beta^{\tau}\right)} \int_{0}^{1} \frac{1}{\sqrt{\psi(x)}} d x+1\right\}=o\left(n^{-1} \beta^{-1 / 2}\right) .
$$




\section{References}

Amoroso, L. (1925) "Ricerche intorno alla curva dei redditi", Annali di Matematica Pura ed Applicata, 2, 123-159.

Birnbaum, Z. W. and Saunders, S. C. (1969) "A new family of life distributions", Journal of Applied Probability, 6, 319-327.

Chen, S. X. (1999) "Beta kernel estimators for density functions", Computational Statistics and Data Analysis, 31, 131-145.

Chen, S. X. (2000) "Probability density function estimation using gamma kernels", Annals of the Institute of Statistical Mathematics, 52, 471-480.

Gautschi, W. (1962) "On inverses of Vandermonde and confluent Vandermonde matrices", $N u$ merische Mathematik, 4, 117-123.

Hirukawa, M. (2010) "Nonparametric multiplicative bias correction for kernel-type density estimation on the unit interval", Computational Statistics and Data Analysis, 54, 473-495. Correction: (2016), 95, 240-242.

Hirukawa, M. and Sakudo, M. (2014) "Nonnegative bias reduction methods for density estimation using asymmetric kernels", Computational Statistics and Data Analysis, 75, 112-123.

Hirukawa, M. and Sakudo, M. (2015) "Family of the generalised gamma kernels: a generator of asymmetric kernels for nonnegative data", Journal of Nonparametric Statistics, 27, 41-63.

Igarashi, G. (2016a) "Bias reductions for beta kernel estimation", Journal of Nonparametric Statistics, 28, 1-30.

Igarashi, G. (2016b) "Weighted log-normal kernel density estimation", Communications in Statistics - Theory and Methods, 45, 6670-6687.

Igarashi, G. and Kakizawa, Y. (2014a) "On improving convergence rate of Bernstein polynomial density estimator", Journal of Nonparametric Statistics, 26, 61-84.

Igarashi, G. and Kakizawa, Y. (2014b) "Re-formulation of inverse Gaussian, reciprocal inverse Gaussian, and Birnbaum-Saunders kernel estimators", Statistics and Probability Letters, 84, $235-246$.

Igarashi, G. and Kakizawa, Y. (2015) "Bias corrections for some asymmetric kernel estimators", Journal of Statistical Planning and Inference, 159, 37-63.

Igarashi, G. and Kakizawa, Y. (2018a) "Generalised gamma kernel density estimation for nonnegative data and its bias reduction", Journal of Nonparametric Statistics, 30, 598-639.

Igarashi, G. and Kakizawa, Y. (2018b) "Limiting bias-reduced Amoroso kernel density estimators for nonnegative data", Communications in Statistics - Theory and Methods, 47, 4905-4937.

Igarashi, G. and Kakizawa, Y. (2018c) "Multiplicative bias correction for asymmetric kernel density estimators revisited", Department of Policy and Planning Sciences Discussion Paper Series No. 1355, University of Tsukuba. 
Jin, X. and Kawczak, J. (2003) "Birnbaum-Saunders and lognormal kernel estimators for modelling durations in high frequency financial data", Annals of Economics and Finance, 4, 103124.

Jones, M. C. (1993) "Simple boundary correction for kernel density estimation", Statistics and Computing, 3, 135-146.

Jones, M. C. and Foster, P. J. (1993) "Generalized jackknifing and higher order kernels", Journal of Nonparametric Statistics, 3, 81-94.

Jones, M. C., Linton, O. and Nielsen, J. P. (1995) "A simple bias reduction method for density estimation", Biometrika, 82, 327-338.

Jørgensen, B. (1982) Statistical Properties of the Generalized Inverse Gaussian Distribution, New York: Springer-Verlag.

Jørgensen, B., Seshadri, V. and Whitmore, G. A. (1991) "On the mixture of the inverse Gaussian distribution with its complementary reciprocal", Scandinavian Journal of Statistics, 18, 77-89.

Kakizawa, Y. (2018) "Nonparametric density estimation for nonnegative data, using symmetrical-based inverse and reciprocal inverse Gaussian kernels through dual transformation", Journal of Statistical Planning and Inference, 193, 117-135.

Kakizawa, Y. and Igarashi, G. (2017) "Inverse gamma kernel density estimation for nonnegative data", Journal of the Korean Statistical Society, 46, 194-207.

Leblanc, A. (2010) "A bias-reduced approach to density estimation using Bernstein polynomials", Journal of Nonparametric Statistics, 22, 459-475.

Marchant, C., Bertin, K., Leiva, V. and Saulo, H. (2013) "Generalized Birnbaum-Saunders kernel density estimators and an analysis of financial data", Computational Statistics and Data Analysis, 63, 1-15.

Marron, J. S. and Ruppert, D. (1994) "Transformations to reduce boundary bias in kernel density estimation", Journal of the Royal Statistical Society, Series B, 56, 653-671.

Nadaraya, E. A. (1974) "On the integral mean square error of some nonparametric estimates for the density function", Theory of Probability and Its Applications, 19, 133-141.

Parzen, E. (1962) "On estimation of a probability density function and mode", The Annals of Mathematical Statistics, 33, 1065-1076.

Rosenblatt, M. (1956) "Remarks on some nonparametric estimates of a density function", The Annals of Mathematical Statistics, 27, 832-837.

Saulo, H., Leiva, V., Ziegelmann, F. A. and Marchant, C. (2013) "A nonparametric method for estimating asymmetric densities based on skewed Birnbaum-Saunders distributions applied to environmental data", Stochastic Environmental Research and Risk Assessment, 27, 14791491.

Scaillet, O. (2004) "Density estimation using inverse and reciprocal inverse Gaussian kernels", Journal of Nonparametric Statistics, 16, 217-226. 
Schucany, W. R. and Sommers, J. P. (1977) "Improvement of kernel type density estimators", Journal of the American Statistical Association, 72, 420-423.

Stacy, E. W. (1962) "A generalization of the gamma distribution", The Annals of Mathematical Statistics, 33, 1187-1192.

Stacy, E. W. and Mihram, G. A. (1965) "Parameter estimation for a generalized gamma distribution", Technometrics, 7, 349-358.

Terrell, G. R. and Scott, D. W. (1980) "On improving convergence rates for nonnegative kernel density estimators", The Annals of Statistics, 8, 1160-1163.

Tweedie, M. C. K. (1957) "Statistical properties of inverse Gaussian distributions. I.", The Annals of Mathematical Statistics, 28, 362-377.

Wand, M. P. and Schucany, W. R. (1990) "Gaussian-based kernels", Canadian Journal of Statistics, 18, 197-204.

Zhang, S., Karunamuni, R. J. and Jones, M. C. (1999) "An improved estimator of the density function at the boundary", Journal of the American Statistical Association, 94, 1231-1241.

Zougab, N. and Adjabi, S. (2016) "Multiplicative bias correction for generalized BirnbaumSaunders kernel density estimators and application to nonnegative heavy tailed data", Journal of the Korean Statistical Society, 45, 51-63. 